\title{
E-Orbit Functions
}

\author{
Anatoliy U. KLIMYK ${ }^{\dagger}$ and Jiri PATERA $\ddagger$ \\ $\dagger$ Bogolyubov Institute for Theoretical Physics, 14-b Metrologichna Str., Kyiv 03680, Ukraine \\ E-mail: aklimyk@bitp.kiev.ua \\ ¥ Centre de Recherches Mathématiques, Université de Montréal, \\ C.P.6128-Centre ville, Montréal, H3C 3J7, Québec, Canada \\ E-mail: patera@crm.umontreal.ca
}

Received December 20, 2007; Published on-line January 05, 2008

Original article is available at http://www.emis.de/journals/SIGMA/2008/002/

\begin{abstract}
We review and further develop the theory of $E$-orbit functions. They are functions on the Euclidean space $E_{n}$ obtained from the multivariate exponential function by symmetrization by means of an even part $W_{e}$ of a Weyl group $W$, corresponding to a CoxeterDynkin diagram. Properties of such functions are described. They are closely related to symmetric and antisymmetric orbit functions which are received from exponential functions by symmetrization and antisymmetrization procedure by means of a Weyl group $W$. The $E$-orbit functions, determined by integral parameters, are invariant with respect to even part $W_{e}^{\text {aff }}$ of the affine Weyl group corresponding to $W$. The $E$-orbit functions determine a symmetrized Fourier transform, where these functions serve as a kernel of the transform. They also determine a transform on a finite set of points of the fundamental domain $F^{e}$ of the group $W_{e}^{\text {aff }}$ (the discrete $E$-orbit function transform).
\end{abstract}

Key words: E-orbit functions; orbits; products of orbits; symmetric orbit functions; $E$-orbit function transform; finite $E$-orbit function transform; finite Fourier transforms

2000 Mathematics Subject Classification: 33-02; 33E99; 42B99; 42C15; 58C40

\section{Introduction}

In [1] and [2] it was initiated a study of orbit functions which are closely related to finite groups $W$ of geometric symmetries generated by reflection transformations $r_{i}$ (that is, such that $r_{i}^{2}=1$ ), $i=1,2, \ldots, n$, of the $n$-dimensional Euclidean space $E_{n}$ with respect to $(n-1)$-dimensional subspaces containing the origin. In fact, orbit functions are multivariate exponential functions symmetrized or antisymmetrized by means of a Weyl group $W$ of a semisimple Lie algebra or symmetrized by means of its subgroup $W_{e}$ consisting of even elements of $W$. Orbit functions on the 2-dimensional Euclidean space $E_{2}$, invariant or anti-invariant with respect to $W$, were considered in detail in $[3,4,5,6]$. A detailed description of symmetric and antisymmetric orbit functions on any Euclidean space $E_{n}$ is given in [7] and in [8]. Orbit functions on $E_{2}$, invariant with respect to $W_{e}$ are studied in [9].

The important peculiarity of orbit functions is a possibility of their discretization [10], which is made by using the results of paper [11]. This possibility makes orbit functions useful for applications. In particular, on this way multivariate discrete Fourier transform and multivariate discrete sine and cosine transforms are received (see $[8,12,13])$.

In order to obtain a symmetric orbit function we take a point $\lambda \in E_{n}$ and act upon $\lambda$ by all elements of the group $W$. If $O(\lambda)$ is the $W$-orbit of the point $\lambda$, that is, the set of all different points of the form $w \lambda, w \in W$, then the symmetric orbit function, determined by $\lambda$, coincides with

$$
\phi_{\lambda}(x)=\sum_{\mu \in O(\lambda)} e^{2 \pi \mathrm{i}\langle\mu, x\rangle},
$$


where $\langle\mu, x\rangle$ is the scalar product on $E_{n}$. These functions are invariant with respect to the action by elements of the group $W: \phi_{\lambda}(w x)=\phi_{\lambda}(x), w \in W$. If $\lambda$ is an integral point of $E_{n}$, then $\phi_{\lambda}(x)$ is invariant with respect to the affine Weyl group $W^{\text {aff }}$ corresponding to $W$.

Symmetry is the main property of symmetric orbit functions which make them useful in applications. Being a modification of monomial symmetric functions, they are directly related to the theory of symmetric (Laurent) polynomials $[14,15,16,17]$ (see Section 11 in [7]).

Symmetric orbit functions $\phi_{\lambda}(x)$ for integral $\lambda$ are closely related to the representation theory of compact groups $G$. In particular, they were effectively used for different calculations in representation theory $[18,19,20,21,22]$. They are constituents of traces (characters) of irreducible unitary representations of $G$.

Antisymmetric orbit functions are given by

$$
\varphi_{\lambda}(x)=\sum_{w \in W}(\operatorname{det} w) e^{2 \pi \mathrm{i}\langle w \lambda, x\rangle}, \quad x \in E_{n},
$$

where $\lambda$ is an element, which does not lie on a wall of a Weyl chamber, and det $w$ is a determinant of the transformation $w$ (it is equal to 1 or -1 , depending on either $w$ is a product of even or odd number of reflections). The orbit functions $\varphi_{\lambda}$ have many properties that the symmetric orbit functions $\phi_{\lambda}$ do. However, antisymmetry leads to some new properties which are useful for applications [6]. For integral $\lambda$, antisymmetric orbit functions are closely related to characters of irreducible representations of the corresponding compact Lie group $G$. Namely, the character $\chi_{\lambda}$ of the irreducible representation $T_{\lambda}, \lambda \in P_{+}$, coincides with $\varphi_{\lambda+\rho} / \varphi_{\rho}$, where $\rho$ is the half-sum of positive roots related to the Weyl group $W$.

A symmetric (antisymmetric) orbit function is the exponential function $e^{2 \pi \mathrm{i}\langle\lambda, x\rangle}$ on $E_{n}$ symmetrized (antisymmetrized) by means of the group $W$. For each transformation group $W$, the symmetric (antisymmetric) orbit functions, characterized by integral $\lambda$, form a complete basis in the space of symmetric (antisymmetric) with respect to $W$ polynomials in $e^{2 \pi \mathrm{i} x_{j}}, j=1,2, \ldots, n$, or an orthogonal basis in the Hilbert space obtained by closing this space of polynomials with respect to an appropriate scalar product.

Orbit functions $\phi_{\lambda}(x)$ (or $\varphi_{\lambda}(x)$ ), when $\lambda$ runs over integral elements, determine the so-called symmetric (antisymmetric) orbit function transform, which is a symmerization (antisymmetrization) of the usual Fourier series expansion on $E_{n}$. If $\lambda$ runs over the dominant Weyl chamber in the space $E_{n}$, then $\phi_{\lambda}(x)$ (or $\varphi_{\lambda}(x)$ ) determine a symmetric (antisymmetric) orbit function transform, which is a symmetrization (antisymmetrization) of the usual continuous Fourier expansion in $E_{n}$ (that is, of the Fourier integral).

The Fourier transform on $\mathbb{R}$ leads to the discrete Fourier transform on grids. In the same way the symmetric and antisymmetric orbit function transforms lead to discrete analogues of these transforms (which are generalizations of the discrete cosine and sine transforms, respectively, [23]). These discrete transforms are useful in many things related to discretization (see $[3,4,5]$ ). Construction of the discrete orbit function transforms are fulfilled by means of the results of paper [11].

Symmetric orbit functions are a generalization of the cosine function, whereas antisymmetric orbit functions are a generalization of the sine function. There appears a natural question: What is a generalization of the exponential function of one variable? This generalization is given by orbit functions symmetric with respect to the subgroup $W_{e}$ of even elements in $W$.

Our goal in this paper is to give in a full generality the theory of orbit functions symmetric with respect to the group $W_{e}$. We shall call these functions $E$-orbit functions, since they are an analogue of the well-known exponential function (since symmetric and antisymmetric orbit functions $\phi_{\lambda}$ and $\varphi_{\lambda}$ are generalizations of the cosine and sine functions, they are often called as $C$-functions and $S$-functions, respectively). This paper is a natural continuation of our papers [7] 
and [8]. Under our exposition we use the results of papers [2] and [10], where $E$-orbit functions are defined.

Roughly speaking, E-orbit functions are related to symmetric and antisymmetric orbit functions in the same way as the exponential function in one variable is related to the sine and cosine functions.

$E$-orbit functions are symmetric with respect to the subgroup $W_{e}$ of the Weyl group $W$, that is, $E_{\lambda}(w x)=E_{\lambda}(x)$ for any $w \in W_{e}$. The subgroup $W_{e}$ is of index 2 in $W$, that is $\left|W / W_{e}\right|=2$, where $|X|$ denote a number of elements in the corresponding set $X$. This means that $E$-orbit functions are determined not only for $\lambda$ from dominant Weyl chamber $D_{+}$(as in the case of symmetric orbit functions), but also for elements from the set $r_{i} D_{+}$, where $r_{i}$ is a fixed reflection from $W$.

If $\lambda$ is an integral element, then the corresponding $E$-orbit function $E_{\lambda}(x)$ is symmetric also with respect to elements of the affine Weyl group $W_{e}^{\text {aff }}$, corresponding to the group $W_{e}$ (in fact, the group $W_{e}^{\text {aff }}$ consists of even elements of the whole affine Weyl group $W^{\text {aff }}$, corresponding to the Weyl group $W$ ).

Symmetry with respect to $W_{e}^{\text {aff }}$ is a main property of $E$-orbit functions with integral $\lambda$. Because of this symmetry, it is enough to determine $E$-orbit functions only on the fundamental domain $F\left(W_{e}^{\text {aff }}\right)$ of the group $W_{e}^{\text {aff }}$ (if $\lambda$ is integral). This fundamental domain consists of two fundamental domains of the whole affine Weyl group $W^{\text {aff }}$.

When the group $W$ is a direct product of its subgroups, say $W=W_{1} \times W_{2}$, then $W_{e}=$ $\left(W_{1}\right)_{e} \times\left(W_{2}\right)_{e}$. In this case $E$-orbit functions of $W_{e}$ are products of $E$-orbit functions of $\left(W_{1}\right)_{e}$ and $\left(W_{2}\right)_{e}$. Hence it suffices to carry out our considerations for groups $W_{e}$ which cannot be represented as a product of its subgroups (that is, for such $W$ for which a corresponding CoxeterDynkin diagram is connected).

$E$-orbit functions with integral $\lambda$ determine the so-called $E$-orbit function transform on the fundamental domain $F\left(W_{e}^{\text {aff }}\right)$ of the group $W_{e}^{\text {aff }}$. It is an expansion of functions on $F\left(W_{e}^{\text {aff }}\right)$ in these $E$-orbit functions. $E$-orbit functions $E_{\lambda}(x)$ with $\lambda$ from $E_{n}$ determine $E$-orbit function transform on the fundamental domain $F\left(W_{e}\right)$ of the group $W_{e}$. It is an analogue of the usual integral Fourier transform.

$E$-orbit functions determine also the discrete $E$-orbit function transforms. They are transforms on grids of the domain $F\left(W_{e}^{\text {aff }}\right)$. These transforms are an analogue of the usual discrete Fourier transforms.

We need for our exposition a general information on Weyl groups, affine Weyl groups and root systems. We have given this information in [7] and [8]. In order to make this paper selfcontained we repeat shortly a part of that information in Section 2. In this section we also describe even Weyl groups and affine even Weyl groups.

In Section 3 we define and study $W_{e}$-orbits. It is shown how $W_{e^{-}}$orbits are related to $W$-orbits. Each $W$-orbit is a $W_{e^{-}}$-orbit or consists of two $W_{e^{-}}$orbit. To each $W_{e^{-}}$orbit there corresponds an $E$-orbit function. $W_{e}$-orbits are parametrized by elements of even dominant Weyl chamber $D_{+}^{e}$.


given. All $W_{e}$-orbits of $A_{3}, B_{3}$ and $C_{3}$ are derived. It is proposed to describe points of $W_{e}$-orbits of $A_{n}, B_{n}, C_{n}$ and $D_{n}$ by means of orthogonal coordinates. Then elements of the group $W_{e}$ and $W_{e}$-orbits are described in a simple way. Section 3 contains also a description of fundamental domains for the groups $W_{e}^{\text {aff }}\left(A_{n}\right), W_{e}^{\text {aff }}\left(B_{n}\right), W_{e}^{\text {aff }}\left(C_{n}\right)$, and $W_{e}^{\text {aff }}\left(D_{n}\right)$.

Section 4 is devoted to description of $E$-orbit functions. $E$-orbit functions, corresponding to Coxeter-Dynkin diagrams, containing only two nodes, are given in an explicit form. In this section we also give explicit formulas for $E$-orbit functions, corresponding to the cases $A_{n}, B_{n}$, $C_{n}$ and $D_{n}$, in the corresponding orthogonal coordinate systems.

In Section 5, properties of $E$-orbit functions are derived. If $\lambda$ is integral, then a main property of the $E$-orbit function $E_{\lambda}(x)$ is an invariance with respect to the affine even Weyl group $W_{e}^{\text {aff }}$. 
Relation of $E$-orbit functions to symmetric and antisymmetric orbit functions (that is, to $W$ orbit functions) is described.

$E$-orbit functions $E_{\lambda}(x)$ with integral $\lambda$ are orthogonal on the closure of the fundamental domain of the group $W_{e}^{\text {aff }}$. This orthogonality is given in Section 5 .

$E$-orbit functions are solutions of the corresponding Laplace equation. This description is exposed in Section 5. E-orbit functions also are solutions of some other differential equations.

In Section 6 we consider expansions of products of $E$-orbit functions into a sum of $E$-orbit functions. These expansions are closely related to properties of $W_{e}$-orbits, namely, the expansions are reduced to decomposition of products of $W_{e}$-orbits into separate $W_{e}$-orbits. In

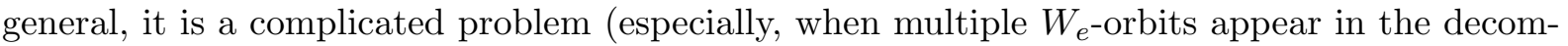

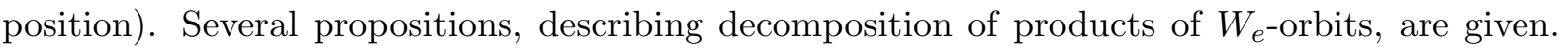
Many examples for expansions in the case of Coxeter-Dynkin diagrams $A_{2}, C_{2}$, and $G_{2}$ are considered.

Section 7 is devoted to expansion of $W_{e}$-orbit functions into a sum of $W_{e}^{\prime}$-orbit functions, where $W_{e}^{\prime}$ is an even Weyl subgroup of the even Weyl group $W_{e}$. The cases of restriction of $A_{n}$ to $A_{n-1}$, of $B_{n}$ to $B_{n-1}$, of $C_{n}$ to $C_{n-1}$, and of $D_{n}$ to $D_{n-1}$ are described in detail.

In Section 8 we expose $E$-orbit function transforms. There are two types of such transforms. The first one is an analogue of the expansion into Fourier series (it is an expansion on the fundamental domain of the group $W_{e}^{\text {aff }}$ ) and the second one is an analogue of the Fourier integral transform (it is an expansion on the even dominant Weyl chamber).

In Section 9 a description of a $W_{e}$-generalization of the multi-dimensional finite Fourier transforms is given. This generalization is connected with grids on the corresponding fundamental domains for the affine even Weyl groups $W_{e}^{\text {aff }}$. These grids are determined by a positive integer $M$. To each such an integer there corresponds a grid on the fundamental domain. Examples of such grids for $A_{2}, C_{2}$ and $G_{2}$ are given.

Section 10 is devoted to exposition of $W_{e}$-symmetric functions, which are symmetric analogues of special functions of mathematical physics or orthogonal polynomials. In particular, we find eigenfunctions of the $W_{e}$-orbit function transforms. These eigenfunctions are connected with classical Hermite polynomials.

\section{Root systems and Weyl groups}

\subsection{Coxeter-Dynkin diagrams and simple roots}

We need finite transformation groups $W$, acting on the $n$-dimensional Euclidean space $E_{n}$, which are generated by reflections $r_{i}, i=1,2, \ldots, n$ (that is, $r_{i}^{2}=1$ ); the theory of such groups see, for example, in [24] and [25]. We are interested in those groups $W$ which are Weyl groups of semisimple Lie groups (semisimple Lie algebras). It is well-known that such Weyl groups together with the corresponding systems of reflections $r_{i}, i=1,2, \ldots, n$, are determined by Coxeter-Dynkin diagrams. There are 4 series of simple Lie algebras and 5 separate simple Lie algebras, which uniquely determine their Weyl groups $W$. These algebras are denoted as

$$
A_{n}(n \geq 1), B_{n}(n \geq 3), C_{n}(n \geq 2), D_{n}(n \geq 4), E_{6}, E_{7}, E_{8}, F_{4}, G_{2} \text {. }
$$

To these simple Lie algebras there correspond connected Coxeter-Dynkin diagrams.

To semisimple Lie algebras (they are direct sums of simple Lie subalgebras) there correspond Coxeter-Dynkin diagrams, which consist of connected parts, corresponding to simple Lie subalgebras; these parts are not connected with each other (a description of the correspondence between simple Lie algebras and Coxeter-Dynkin diagrams see, for example, in [26]). Thus, we describe only Coxeter-Dynkin diagrams, corresponding to simple Lie algebras. They are of the form 



$G_{2} \stackrel{1}{\rightleftharpoons}$

A diagram determines a certain non-orthogonal basis $\left\{\alpha_{1}, \alpha_{2}, \ldots, \alpha_{n}\right\}$ in the Euclidean space $E_{n}$. Each node of a diagram is associated with a basis vector $\alpha_{k}$, called a simple root. A direct link between two nodes indicates that the corresponding basis vectors are not orthogonal. Conversely, an absence of a direct link between nodes implies orthogonality of the corresponding vectors. Single, double, and triple links indicate that the relative angles between the two simple roots are $2 \pi / 3,3 \pi / 4,5 \pi / 6$, respectively. There can be only two cases: all simple roots are of the same length or there are only two different lengths of simple roots. In the first case all simple roots are denoted by white nodes. In the case of two lengths, shorter roots are denoted by black nodes and longer ones by white nodes. Lengths of roots are determined uniquely up to a common constant. For the cases $B_{n}, C_{n}$, and $F_{4}$, the squared longer root length is double the squared shorter root length. For $G_{2}$, the squared longer root length is triple the squared shorter root length. Simple roots of the same length are orthogonal to each other or an angle between them is $2 \pi / 3$. A number of simple roots is called a rank of the corresponding Lie algebra.

To each Coxeter-Dynkin diagram there corresponds a Cartan matrix $M$, consisting of the entries

$$
M_{j k}=\frac{2\left\langle\alpha_{j}, \alpha_{k}\right\rangle}{\left\langle\alpha_{k}, \alpha_{k}\right\rangle}, \quad j, k \in\{1,2, \ldots, n\},
$$

where $\langle x, y\rangle$ denotes the scalar product of $x, y \in E_{n}$. Cartan matrices of simple Lie algebras are given in many places (see, for example, [27]). For ranks 2 and 3 they are of the form:

$$
\begin{aligned}
& A_{2}:\left(\begin{array}{cc}
2 & -1 \\
-1 & 2
\end{array}\right), \quad C_{2}:\left(\begin{array}{cc}
2 & -1 \\
-2 & 2
\end{array}\right), \quad G_{2}:\left(\begin{array}{cc}
2 & -3 \\
-1 & 2
\end{array}\right) \text {, } \\
& A_{3}:\left(\begin{array}{ccc}
2 & -1 & 0 \\
-1 & 2 & -1 \\
0 & -1 & 2
\end{array}\right), \quad B_{3}:\left(\begin{array}{ccc}
2 & -1 & 0 \\
-1 & 2 & -2 \\
0 & -1 & 2
\end{array}\right), \quad C_{3}:\left(\begin{array}{ccc}
2 & -1 & 0 \\
-1 & 2 & -1 \\
0 & -2 & 2
\end{array}\right) \text {. }
\end{aligned}
$$

Lengths of the basis vectors $\alpha_{i}$ are fixed by the corresponding Coxeter-Dynkin diagram up to a constant. We adopt the standard choice in the Lie theory, namely

$$
\langle\alpha, \alpha\rangle=2
$$

for all simple roots of $A_{n}, D_{n}, E_{6}, E_{7}, E_{8}$ and for the longer simple roots of $B_{n}, C_{n}, F_{4}, G_{2}$.

\subsection{Weyl group and even Weyl group}

A Coxeter-Dynkin diagram determines uniquely the corresponding transformation group $W$ of the Euclidean space $E_{n}$, generated by reflections $r_{i}, i=1,2, \ldots, n$. These reflections correspond 
to simple roots $\alpha_{i}, i=1,2, \ldots, n$. Namely, the transformation $r_{i}$ corresponds to the simple root $\alpha_{i}$ and is the reflection with respect to $(n-1)$-dimensional linear subspace (hyperplane) of $E_{n}$ (containing the origin), orthogonal to $\alpha_{i}$. Such reflections are given by the formula

$$
r_{i} x=x-\frac{2\left\langle x, \alpha_{i}\right\rangle}{\left\langle\alpha_{i}, \alpha_{i}\right\rangle} \alpha_{i}, \quad i=1,2, \ldots, n, \quad x \in E_{n}
$$

Each reflection $r_{i}$ can be thought as attached to the $i$-th node of the corresponding diagram.

A finite group $W$, generated by the reflections $r_{i}, i=1,2, \ldots, n$, is called a Weyl group, corresponding to a given Coxeter-Dynkin diagram. If a Weyl group $W$ corresponds to a CoxeterDynkin diagram of a simple Lie algebra $L$, then this Weyl group is often denoted by $W(L)$. Properties of Weyl groups are well known (see [24] and [25]). The orders (numbers of elements) of Weyl groups are given by the formulas

$$
\begin{aligned}
& \left|W\left(A_{n}\right)\right|=(n+1) !, \quad\left|W\left(B_{n}\right)\right|=\left|W\left(C_{n}\right)\right|=2^{n} n !, \quad\left|W\left(D_{n}\right)\right|=2^{n-1} n !, \\
& \left|W\left(E_{6}\right)\right|=51840, \quad\left|W\left(E_{7}\right)\right|=2903040, \quad\left|W\left(E_{8}\right)\right|=696729600, \\
& \left|W\left(F_{4}\right)\right|=1152, \quad\left|W\left(G_{2}\right)\right|=12 .
\end{aligned}
$$

In particular,

$$
\left|W\left(A_{2}\right)\right|=6, \quad\left|W\left(C_{2}\right)\right|=8, \quad\left|W\left(A_{3}\right)\right|=24, \quad\left|W\left(B_{3}\right)\right|=\left|W\left(C_{3}\right)\right|=48 .
$$

Elements of the Weyl groups are linear transformations of the Euclidean space $E_{n}$. To these transformations there correspond in an orthonormal basis of $E_{n}$ the corresponding $n \times n$ matrices. Since these transformations are orthogonal, then determinants of these matrices are +1 or -1 . We say that a transformation $w \in W$ is even if $\operatorname{det} w=1$ and odd if $\operatorname{det} w=-1$. Clearly, for reflections $r_{\alpha}$ corresponding to roots $\alpha$ we have $\operatorname{det} r_{\alpha}=-1$. If $w \in W$ is a product of even (odd) number of reflections, then $\operatorname{det} w=1(\operatorname{det} w=-1)$.

The set of all elements $w \in W$ with $\operatorname{det} w=1$ constitute a subgroup of $W$ which will be denoted by $W_{e}$. One says that it is a subgroup of even elements of $W$. Moreover, $W_{e}$ is a normal subgroup of $W$, that is, $w W_{e} w^{-1}=W_{e}$ for any $w \in W$. The group $W_{e}$ is a basic group for definition of $E$-orbit functions.

Elements of $W$, which do not belong to $W_{e}$, are called odd. The number of even elements in $W$ is equal to the number of odd elements, that is, $\left|W / W_{e}\right|=2$. In particular, we have

$$
\begin{aligned}
& \left|W_{e}\left(A_{2}\right)\right|=3, \quad\left|W_{e}\left(C_{2}\right)\right|=4, \quad\left|W_{e}\left(G_{2}\right)\right|=6, \quad\left|W_{e}\left(A_{3}\right)\right|=12, \\
& \left|W_{e}\left(B_{3}\right)\right|=\left|W_{e}\left(C_{3}\right)\right|=24 .
\end{aligned}
$$

The Weyl groups $W\left(B_{3}\right)$ and $W\left(C_{3}\right)$ are isomorphic. For this reason, the even Weyl groups $W_{e}\left(B_{3}\right)$ and $W_{e}\left(C_{3}\right)$ are isomorphic.

Elements of $W_{e}$ are orthogonal transformations of $E_{n}$ with a unit determinant. Therefore, $W_{e}$ is a finite subgroup of the rotation group $S O(n)$ of $E_{n}$. That is, the group $W_{e}$ consists of rotations of the space $E_{n}$. In particular, for rank 2 case the even Weyl groups consist of rotations of a plane:

$$
\begin{aligned}
& W_{e}\left(A_{2}\right)=\{1, \operatorname{rot}(2 \pi / 3), \operatorname{rot}(4 \pi / 3)\}, \\
& W_{e}\left(C_{2}\right)=\{1, \operatorname{rot}(\pi / 2), \operatorname{rot}(\pi), \operatorname{rot}(3 \pi / 2)\}, \\
& W_{e}\left(G_{2}\right)=\{1, \operatorname{rot}(k \pi / 3), k=1,2,3,4,5\} .
\end{aligned}
$$




\subsection{Root and weight lattices}

A Coxeter-Dynkin diagram determines a system of simple roots in the Euclidean space $E_{n}$. Acting by elements of the Weyl group $W$ upon simple roots we obtain a finite system of vectors, which is invariant with respect to $W$. A set of all these vectors is called a system of roots associated with a given Coxeter-Dynkin diagram. It is denoted by $R$.

It is proved (see, for example, [26]) that roots of $R$ are linear combinations of simple roots with integral coefficients. Moreover, there exist no roots, which are linear combinations of $\alpha_{i}$, $i=1,2, \ldots, n$, both with positive and negative coefficients. Therefore, the set of roots $R$ can be represented as a union $R=R_{+} \cup R_{-}$, where $R_{+}$(respectively $R_{-}$) is the set of roots which are linear combinations of simple roots with positive (negative) coefficients. The set $R_{+}$(the set $R_{-}$) is called a set of positive (negative) roots.

As mentioned above, a set of roots $R$ is invariant under the action of elements of the Weyl group $W(R)$. However, $w R_{+} \neq R_{+}$if $w$ is not a trivial element of $W$.

Let $X_{\alpha}$ be the $(n-1)$-dimensional linear subspace (hyperplane) of $E_{n}$ (containing the origin) which is orthogonal to the root $\alpha$. The hyperplane $X_{\alpha}$ consists of all points $x \in E_{n}$ such that $\langle x, \alpha\rangle=0$. Clearly, $X_{\alpha}=X_{-\alpha}$. The set of reflections with respect to $X_{\alpha}, \alpha \in R_{+}$, coincides with the set of all reflections of the corresponding Weyl group $W$.

The subspaces $X_{\alpha}, \alpha \in R_{+}$, split the Euclidean space $E_{n}$ into connected parts which are called Weyl chambers. (We assume that boundaries of Weyl chambers belong to the corresponding chambers. Therefore, Weyl chambers can have common points; they belong to boundaries of the corresponding chambers.) A number of Weyl chambers coincides with the number of elements of the Weyl group $W$. Elements of the Weyl group permute Weyl chambers. A part of a Weyl chamber, which belongs to some hyperplane $X_{\alpha}$ is called a wall of this Weyl chamber. If for some element $x$ of a Weyl chamber we have $\langle x, \alpha\rangle=0$ for some root $\alpha$, then this point belongs to a wall. The Weyl chamber consisting of points $x$ such that

$$
\left\langle x, \alpha_{i}\right\rangle \geq 0, \quad i=1,2, \ldots, n,
$$

is called the dominant Weyl chamber. It is denoted by $D_{+}$. Elements of $D_{+}$are called dominant. If $\left\langle x, \alpha_{i}\right\rangle>0, i=1,2, \ldots, n$, then $x$ is called strictly dominant element.

If we act by elements of the even subgroup $W_{e}$ of $W$ upon a fixed Weyl chamber, then we do not obtain all Weyl chambers. In order to have transitive action of $W_{e}$ on parts of the Euclidean space $E_{n}$, we have to split $E_{n}$ into a parts larger than Weyl chambers. In order to obtain such parts, we take the dominant Weyl chamber $D_{+}$and act upon it by one of the reflections $r_{\alpha}$, where $\alpha$ is a root. Denote the union $D_{+} \cup r_{\alpha} D_{+}$(where each point is taken only once) by $D_{+}^{e}$. Then acting upon $D_{+}^{e}$ by elements of $W_{e}$ we cover the whole Euclidean space $E_{n}$. The domains $w D_{+}^{e}, w \in W_{e}$, are called even Weyl chambers. The procedure of splitting of $E_{n}$ into even Weyl chambers is not unique. It depends on the reflection $r_{\alpha}$ taken for obtaining the first even Weyl chambers. For different roots $\alpha$ sets of even Weyl chambers are different. However, for each fixed root $\alpha$ the corresponding set of even Weyl chambers is transitive for the group $W_{e}$. The set $D_{+}^{e} \equiv D_{+}^{e}(\alpha)$ is called an even dominant Weyl chamber.

The set $Q$ of all linear combinations

$$
Q=\left\{\sum_{i=1}^{n} a_{i} \alpha_{i} \mid a_{i} \in \mathbb{Z}\right\} \equiv \bigoplus_{i} \mathbb{Z} \alpha_{i}
$$

is called a root lattice corresponding to a given Coxeter-Dynkin diagram. Its subset

$$
Q_{+}=\left\{\sum_{i=1}^{n} a_{i} \alpha_{i} \mid a_{i}=0,1,2, \ldots\right\}
$$

is called a positive root lattice. 
To each root $\alpha \in R$ there corresponds a coroot $\alpha^{\vee}$ defined by the formula

$$
\alpha^{\vee}=\frac{2 \alpha}{\langle\alpha, \alpha\rangle} .
$$

It is easy to see that $\alpha^{\vee \vee}=\alpha$. The set $Q^{\vee}$ of all linear combinations

$$
Q^{\vee}=\left\{\sum_{i=1}^{n} a_{i} \alpha_{i}^{\vee} \mid a_{i} \in \mathbb{Z}\right\} \equiv \bigoplus_{i} \mathbb{Z} \alpha_{i}^{\vee}
$$

is called a coroot lattice corresponding to a given Coxeter-Dynkin diagram. The subset

$$
Q_{+}^{\vee}=\left\{\sum_{i=1}^{n} a_{i} \alpha_{i}^{\vee} \mid a_{i}=0,1,2, \ldots\right\}
$$

is called a positive coroot lattice.

As noted above, the set of simple roots $\alpha_{i}, i=1,2, \ldots, n$, form a basis of the space $E_{n}$. In addition to the $\alpha$-basis, it is convenient to introduce the so-called $\omega$-basis, $\omega_{1}, \omega_{2}, \ldots, \omega_{n}$ (also called the basis of fundamental weights). The two bases are dual to each other in the following sense:

$$
\frac{2\left\langle\alpha_{j}, \omega_{k}\right\rangle}{\left\langle\alpha_{j}, \alpha_{j}\right\rangle} \equiv\left\langle\alpha_{j}^{\vee}, \omega_{k}\right\rangle=\delta_{j k}, \quad j, k \in\{1,2, \ldots, n\} .
$$

The $\omega$-basis (as well as the $\alpha$-basis) is not orthogonal.

Note that the factor $2 /\left\langle\alpha_{j}, \alpha_{j}\right\rangle$ can take only three values. Indeed, with the standard normalization of root lengths (see Subsection 2.1), we have

$$
\begin{aligned}
& \frac{2}{\left\langle\alpha_{k}, \alpha_{k}\right\rangle}=1 \quad \text { for roots of } A_{n}, D_{n}, E_{6}, E_{7}, E_{8}, \\
& \frac{2}{\left\langle\alpha_{k}, \alpha_{k}\right\rangle}=1 \quad \text { for long roots of } B_{n}, C_{n}, F_{4}, G_{2}, \\
& \frac{2}{\left\langle\alpha_{k}, \alpha_{k}\right\rangle}=2 \quad \text { for short roots of } B_{n}, C_{n}, F_{4}, \\
& \frac{2}{\left\langle\alpha_{k}, \alpha_{k}\right\rangle}=3 \quad \text { for short roots of } G_{2} .
\end{aligned}
$$

For this reason, we get

$$
\begin{aligned}
& \alpha_{k}^{\vee}=\alpha_{k} \quad \text { for roots of } A_{n}, D_{n}, E_{6}, E_{7}, E_{8}, \\
& \alpha_{k}^{\vee}=\alpha_{k} \quad \text { for long roots of } B_{n}, C_{n}, F_{4}, G_{2}, \\
& \alpha_{k}^{\vee}=2 \alpha_{k} \quad \text { for short roots of } B_{n}, C_{n}, F_{4}, \\
& \alpha_{k}^{\vee}=3 \alpha_{k} \quad \text { for short roots of } G_{2} .
\end{aligned}
$$

The $\alpha$ - and $\omega$-bases are related by the Cartan matrix (2.1) and by its inverse:

$$
\alpha_{j}=\sum_{k=1}^{n} M_{j k} \omega_{k}, \quad \omega_{j}=\sum_{k=1}^{n}\left(M^{-1}\right)_{j k} \alpha_{k}
$$

For ranks 2 and 3 the inverse Cartan matrices are of the form

$$
A_{2}: \frac{1}{3}\left(\begin{array}{cc}
2 & 1 \\
1 & 2
\end{array}\right), \quad C_{2}:\left(\begin{array}{cc}
1 & 1 / 2 \\
1 & 1
\end{array}\right), \quad G_{2}:\left(\begin{array}{cc}
2 & 3 \\
1 & 2
\end{array}\right),
$$




$$
A_{3}: \frac{1}{4}\left(\begin{array}{lll}
3 & 2 & 1 \\
2 & 4 & 2 \\
1 & 2 & 3
\end{array}\right), \quad B_{3}: \frac{1}{2}\left(\begin{array}{ccc}
2 & 2 & 2 \\
2 & 4 & 4 \\
1 & 2 & 3
\end{array}\right), \quad C_{3}: \frac{1}{2}\left(\begin{array}{lll}
2 & 2 & 1 \\
2 & 4 & 2 \\
2 & 4 & 3
\end{array}\right) .
$$

Later on we need to calculate the scalar product $\langle x, y\rangle$ when $x$ and $y$ are given by coordinates $x_{i}$ and $y_{i}$ in $\omega$-basis. It is given by the formula

$$
\langle x, y\rangle=\frac{1}{2} \sum_{j, k=1}^{n} x_{j} y_{k}\left(M^{-1}\right)_{j k}\left\langle\alpha_{k} \mid \alpha_{k}\right\rangle=x M^{-1} D y^{T}=x S y^{T},
$$

where $D$ is the diagonal matrix $\operatorname{diag}\left(\frac{1}{2}\left\langle\alpha_{1}, \alpha_{1}\right\rangle, \ldots, \frac{1}{2}\left\langle\alpha_{n}, \alpha_{n}\right\rangle\right)$. Matrices $S$, called 'quadratic form matrices', are shown in [27] for all connected Coxeter-Dynkin diagrams.

The sets $P$ and $P_{+}$, defined as

$$
P=\mathbb{Z} \omega_{1}+\cdots+\mathbb{Z} \omega_{n} \supset P_{+}=\mathbb{Z}^{\geq 0} \omega_{1}+\cdots+\mathbb{Z}^{\geq 0} \omega_{n},
$$

are called respectively the weight lattice and the cone of dominant weights. The set $P$ can be characterized as a set of all $\lambda \in E_{n}$ such that

$$
\frac{2\left\langle\alpha_{j}, \lambda\right\rangle}{\left\langle\alpha_{j}, \alpha_{j}\right\rangle}=\left\langle\alpha_{j}^{\vee}, \lambda\right\rangle \in \mathbb{Z}
$$

for all simple roots $\alpha_{j}$. Clearly, $Q \subset P$. Below we shall need also the set $P_{+}^{+}$of dominant weights of $P_{+}$, which do not belong to any Weyl chamber (the set of integral strictly dominant weights). Then $\lambda \in P_{+}^{+}$means that $\left\langle\lambda, \alpha_{i}\right\rangle>0$ for all simple roots $\alpha_{i}$. We have

$$
P_{+}^{+}=\mathbb{Z}^{>0} \omega_{1}+\mathbb{Z}^{>0} \omega_{2}+\cdots+\mathbb{Z}^{>0} \omega_{n}
$$

The smallest dominant weights of $P_{+}$, different from zero, coincide with the elements $\omega_{1}, \omega_{2}$, $\ldots, \omega_{n}$ of the $\omega$-basis. They are called fundamental weights. They are highest weights of fundamental irreducible representations of the corresponding simple Lie algebra $L$.

Through the paper we often use the following notation for weights in $\omega$-basis:

$$
z=\sum_{j=1}^{n} a_{j} \omega_{j}=\left(\begin{array}{llll}
a_{1} & a_{2} \ldots a_{n}
\end{array}\right), \quad a_{1}, \ldots, a_{n} \in \mathbb{Z} .
$$

$$
\begin{aligned}
& \text { If } x=\sum_{j=1}^{n} b_{j} \alpha_{j}^{\vee} \text {, then } \\
& \langle z, x\rangle=\sum_{j=1}^{n} a_{j} b_{j} .
\end{aligned}
$$

\subsection{Highest root and affine root system}

There exists a unique highest (long) root $\xi$ and a unique highest short root $\xi_{s}$. The highest (long) root can be written as

$$
\xi=\sum_{i=1}^{n} m_{i} \alpha_{i}=\sum_{i=1}^{n} m_{i} \frac{\left\langle\alpha_{i}, \alpha_{i}\right\rangle}{2} \alpha_{i}^{\vee} \equiv \sum_{i=1}^{n} q_{i} \alpha_{i}^{\vee} .
$$

The coefficients $m_{i}$ and $q_{i}$ can be viewed as attached to the $i$-th node of the diagram. They are called marks and comarks (see, for example, [27]). In root systems with two lengths of roots, that is, in $B_{n}, C_{n}, F_{4}$ and $G_{2}$, the highest (long) root $\xi$ is of the form

$$
B_{n}: \quad \xi=(010 \ldots 0)=\alpha_{1}+2 \alpha_{2}+2 \alpha_{3}+\cdots+2 \alpha_{n},
$$




$$
\begin{array}{rlrlrl}
C_{n}: & \xi & = & (20 \ldots 0) & =2 \alpha_{1}+2 \alpha_{2}+\cdots+2 \alpha_{n-1}+\alpha_{n} \\
F_{4}: & \xi & & & (1000) & =2 \alpha_{1}+3 \alpha_{2}+4 \alpha_{3}+2 \alpha_{4} \\
G_{2}: & \xi & & (10) & =2 \alpha_{1}+3 \alpha_{2}
\end{array}
$$

For $A_{n}, D_{n}$, and $E_{n}$, all roots are of the same length, hence $\xi_{s}=\xi$. We have

$$
\begin{aligned}
& A_{n}: \quad \xi=(10 \ldots 01)=\alpha_{1}+\alpha_{2}+\cdots+\alpha_{n}, \\
& D_{n}: \quad \xi=(010 \ldots 0)=\alpha_{1}+2 \alpha_{2}+\cdots+2 \alpha_{n-2}+\alpha_{n-1}+\alpha_{n} \text {, } \\
& E_{6}: \quad \xi=(010 \ldots 0)=\alpha_{1}+2 \alpha_{2}+3 \alpha_{3}+2 \alpha_{4}+\alpha_{5}+2 \alpha_{6} \text {, } \\
& E_{7}: \quad \xi=(100 \ldots 0)=2 \alpha_{1}+3 \alpha_{2}+4 \alpha_{3}+3 \alpha_{4}+2 \alpha_{5}+\alpha_{6}+2 \alpha_{7} \text {, } \\
& E_{8}: \quad \xi=(00 \ldots 01)=2 \alpha_{1}+3 \alpha_{2}+4 \alpha_{3}+5 \alpha_{4}+6 \alpha_{5}+4 \alpha_{6}+2 \alpha_{7}+3 \alpha_{8} \text {. }
\end{aligned}
$$

For highest root $\xi$ we have

$$
\xi^{\vee}=\xi
$$

Moreover, if all simple roots are of the same length, then

$$
\alpha_{i}^{\vee}=\alpha_{i}
$$

For this reason,

$$
\left(q_{1}, q_{2}, \ldots, q_{n}\right)=\left(m_{1}, m_{2}, \ldots, m_{n}\right) .
$$

for $A_{n}, D_{n}$ and $E_{n}$. Formulas (2.13)-(2.18) determine these numbers. For short roots $\alpha_{i}$ of $B_{n}$, $C_{n}$ and $F_{4}$ we have $\alpha_{i}^{\vee}=2 \alpha_{i}$. For short root $\alpha_{2}$ of $G_{2}$ we have $\alpha_{2}^{\vee}=3 \alpha_{2}$. For this reason,

$$
\begin{array}{ll}
\left(q_{1}, q_{2}, \ldots, q_{n}\right)=(1,2, \ldots, 2,1) \quad \text { for } & B_{n}, \\
\left(q_{1}, q_{2}, \ldots, q_{n}\right)=(1,1, \ldots, 1,1) \quad \text { for } \quad C_{n} \\
\left(q_{1}, q_{2}, q_{3}, q_{4}\right)=(2,3,2,1) \quad \text { for } & F_{4}, \\
\left(q_{1}, q_{2}\right)=(2,1) \quad \text { for } G_{2} . &
\end{array}
$$

To each root system $R$ there corresponds an extended root system (which is also called an affine root system). It is constructed with the help of the highest root $\xi$ of $R$. Namely, if $\alpha_{1}, \alpha_{2}, \ldots, \alpha_{n}$ is a set of all simple roots, then the roots

$$
\alpha_{0}:=-\xi, \alpha_{1}, \alpha_{2}, \ldots, \alpha_{n}
$$

constitute a set of simple roots of the corresponding extended root system. Taking into account the orthogonality (non-orthogonality) of the root $\alpha_{0}$ to other simple roots, a diagram of an extended root system can be constructed (which is an extension of the corresponding CoxeterDynkin diagram; see, for example, [28]). Note that for all simple Lie algebras (except for $A_{n}$ ) only one simple root is orthogonal to the root $\alpha_{0}$. In the case of $A_{n}$, the two simple roots $\alpha_{1}$ and $\alpha_{n}$ are not orthogonal to $\alpha_{0}$.

\subsection{Affine Weyl group and even affine Weyl group}

We are interested in $E$-orbit functions which are given on the Euclidean space $E_{n}$. These functions are invariant with respect to action by elements of an even Weyl group $W_{e}$, which is a transformation group of $E_{n}$. However, $W_{e}$ does not describe all symmetries of $E$-orbit functions corresponding to weights $\lambda \in P_{+}^{e} \equiv P_{+} \cup r_{\alpha} P_{+}$. A whole group of symmetries of these 
$E$-orbit functions is isomorphic to the even affine Weyl group $W_{e}^{\text {aff }}$ which is an extension of the even Weyl group $W$. To describe the group $W_{e}^{\text {aff }}$ we first define the affine Weyl group $W^{\text {aff }}$.

Let $\alpha_{1}, \alpha_{2}, \ldots, \alpha_{n}$ be simple roots in the Euclidean space $E_{n}$ and let $W$ be the corresponding Weyl group. The group $W$ is generated by reflections $r_{\alpha_{i}}, i=1,2, \ldots, n$. In order to construct the affine Weyl group $W^{\text {aff }}$, corresponding to $W$, we have to add an additional reflection. This reflection is constructed as follows.

We consider the reflection $r_{\xi}$ with respect to the $(n-1)$-dimensional subspace (hyperplane) $X_{n-1}$ containing the origin and orthogonal to the highest (long) root $\xi$, given in (2.8):

$$
r_{\xi} x=x-\frac{2\langle x, \xi\rangle}{\langle\xi, \xi\rangle} \xi
$$

Clearly, $r_{\xi} \in W$. We shift the hyperplane $X_{n-1}$ by the vector $\xi^{\vee} / 2$, where $\xi^{\vee}=2 \xi /\langle\xi, \xi\rangle$. (Note that by (2.18) we have $\xi^{\vee}=\xi$. However, it is convenient here to use $\xi^{\vee}$.) The reflection with respect to the hyperplane $X_{n-1}+\xi^{\vee} / 2$ will be denoted by $r_{0}$. In order to fulfill the transformation $r_{0}$ we have to fulfill the transformation $r_{\xi}$ and then to shift the result by $\xi^{\vee}$, that is,

$$
r_{0} x=r_{\xi} x+\xi^{\vee} .
$$

We have $r_{0} 0=\xi^{\vee}$ and it follows from (2.19) that $r_{0}$ maps $x+\xi^{\vee} / 2$ to

$$
r_{\xi}\left(x+\xi^{\vee} / 2\right)+\xi^{\vee}=x+\xi^{\vee} / 2-\left\langle x, \xi^{\vee}\right\rangle \xi
$$

Therefore,

$$
\begin{aligned}
r_{0}\left(x+\xi^{\vee} / 2\right) & =x+\xi^{\vee} / 2-\frac{2\langle x, \xi\rangle}{\langle\xi, \xi\rangle} \xi=x+\xi^{\vee} / 2-\frac{2\left\langle x, \xi^{\vee}\right\rangle}{\left\langle\xi^{\vee}, \xi^{\vee}\right\rangle} \xi^{\vee} \\
& =x+\xi^{\vee} / 2-\frac{2\left\langle x+\xi^{\vee} / 2, \xi^{\vee}\right\rangle}{\left\langle\xi^{\vee}, \xi^{\vee}\right\rangle} \xi^{\vee}+\frac{2\left\langle\xi^{\vee} / 2, \xi^{\vee}\right\rangle}{\left\langle\xi^{\vee}, \xi^{\vee}\right\rangle} \xi^{\vee} .
\end{aligned}
$$

Denoting $x+\xi^{\vee} / 2$ by $y$ we obtain that $r_{0}$ is given also by the formula

$$
r_{0} y=y+\left(1-\frac{2\left\langle y, \xi^{\vee}\right\rangle}{\left\langle\xi^{\vee}, \xi^{\vee}\right\rangle}\right) \xi^{\vee}=\xi^{\vee}+r_{\xi} y
$$

The element $r_{0}$ does not belongs to $W$ since elements of $W$ do not move the point $0 \in E_{n}$.

The hyperplane $X_{n-1}+\xi^{\vee} / 2$ coincides with the set of points $y$ such that $r_{0} y=y$. It follows from (2.20) that this hyperplane is given by the equation

$$
1=\frac{2\left\langle y, \xi^{\vee}\right\rangle}{\left\langle\xi^{\vee}, \xi^{\vee}\right\rangle}=\langle y, \xi\rangle=\sum_{k=1}^{n} a_{k} q_{k},
$$

where

$$
y=\sum_{k=1}^{n} a_{k} \omega_{k}, \quad \xi=\sum_{k=1}^{n} q_{k} \alpha_{k}^{\vee}
$$

(see $(2.7))$.

A group of transformations of the Euclidean space $E_{n}$ generated by reflections $r_{0}, r_{\alpha_{1}}, \ldots, r_{\alpha_{n}}$ is called the affine Weyl group of the root system $R$ and is denoted by $W^{\text {aff }}$ or by $W_{R}^{\text {aff }}$ (if is necessary to indicate the initial root system), see [28]. Adjoining the reflection $r_{0}$ to the Weyl group $W$ completely changes properties of the group $W^{\text {aff }}$. 
Due to (2.19) and (2.20) for any $x \in E_{n}$ we have

$$
r_{0} r_{\xi} x=r_{0}\left(r_{\xi} x\right)=\xi^{\vee}+r_{\xi} r_{\xi} x=x+\xi^{\vee} .
$$

Clearly, $\left(r_{0} r_{\xi}\right)^{k} x=x+k \xi^{\vee}, k=0, \pm 1, \pm 2, \ldots$, that is, the set of elements $\left(r_{0} r_{\xi}\right)^{k}, k=$ $0, \pm 1, \pm 2, \ldots$, is an infinite commutative subgroup of $W^{\text {aff }}$. This means that (unlike to the Weyl group $W) W^{\text {aff }}$ is an infinite group.

Since $r_{0} 0=\xi^{\vee}$, for any $w \in W$ we have

$$
w r_{0} 0=w \xi^{\vee}=\xi_{w}^{\vee}
$$

where $\xi_{w}^{\vee}$ is a coroot of the same length as the coroot $\xi^{\vee}$. For this reason, $w r_{0}$ is the reflection with respect to the $(n-1)$-hyperplane perpendicular to the root $\xi_{w}^{\vee}$ and containing the point $\xi_{w}^{\vee} / 2$. Moreover,

$$
\left(w r_{0}\right) r_{\xi_{w}} x=x+\xi_{w}^{\vee}
$$

We also have $\left(\left(w r_{0}\right) r_{\xi_{w}}\right)^{k} x=x+k \xi_{w}^{\vee}, k=0, \pm 1, \pm 2, \ldots$ Since $w$ is any element of $W$, then the set $w \xi^{\vee}, w \in W$, coincides with the set of coroots of $R$, corresponding to all long roots of the root system $R$. Thus, the set $W^{\text {aff }} \cdot 0$ coincides with the lattice $Q_{l}^{\vee}$ generated by coroots $\alpha^{\vee}$ taken for all long roots $\alpha$ from $R$.

It is checked for each type of root systems that each coroot $\xi_{s}^{\vee}$ for a short root $\xi_{s}$ of $R$ is a linear combination of coroots $w \xi^{\vee} \equiv \xi_{w}, w \in W$, with integral coefficients, that is, $Q^{\vee}=Q_{l}^{\vee}$. Therefore, The set $W^{\text {aff }} \cdot 0$ coincides with the coroot lattice $Q^{\vee}$ of $R$.

Let $\hat{Q}^{\vee}$ be the subgroup of $W^{\text {aff }}$ generated by the elements

$$
\left(w r_{0}\right) r_{w}, \quad w \in W,
$$

where $r_{w} \equiv r_{\xi_{w}}$ for $w \in W$. Since elements (2.22) pairwise commute with each other (since they are shifts), $\hat{Q}^{\vee}$ is a commutative group. The subgroup $\hat{Q}^{\vee}$ can be identified with the coroot lattice $Q^{\vee}$. Namely, if for $g \in \hat{Q}^{\vee}$ we have $g \cdot 0=\gamma \in Q^{\vee}$, then $g$ is identified with $\gamma$. This correspondence is one-to-one.

The subgroups $W$ and $\hat{Q}^{\vee}$ generate $W^{\text {aff }}$ since a subgroup of $W^{\text {aff }}$, generated by $W$ and $\hat{Q}^{\vee}$, contains the element $r_{0}$. The group $W^{\text {aff }}$ is a semidirect product of its subgroups $W$ and $\hat{Q}^{\vee}$, where $\hat{Q}^{\vee}$ is an invariant subgroup (see Section 5.2 in [7] for details).

We shall need not the whole affine Weyl group $W^{\text {aff }}$ but only its subgroup, constructed on the base of the even Weyl group $W_{e}$. The subgroup $W_{e}^{\text {aff }}$, coinciding with the semidirect product of the even Weyl group $W_{e}$ and $\hat{Q}^{\vee}$, will be called an even affine Weyl group. This subgroup does not contain the reflection $r_{0} \in W^{\text {aff }}$. One says that $W_{e}^{\text {aff }}$ consists of even elements of $W^{\text {aff }}$.

\section{6 $\quad W^{\text {aff }}$-fundamental domain and $W_{e}^{\text {aff }}$-fundamental domain}

An open connected simply connected set $F(G) \subset E_{n}$ is called a fundamental domain for the group $G\left(G=W, W^{\text {aff }}, W_{e}, W_{e}^{\text {aff }}\right)$ if it does not contains equivalent points (that is, points $x$ and $x^{\prime}$ such that $x=w x, w \in G$ ) and if its closure contains at least one point from each $G$-orbit. It is evident that the dominant Weyl chamber $D_{+}$without walls of this chamber is a fundamental domain for the Weyl group $W$. Recall that this domain consists of all points $x=a_{1} \omega_{1}+\cdots+a_{n} \omega_{n} \in E_{n}$ for which

$$
a_{i}=\left\langle x, \alpha_{i}^{\vee}\right\rangle>0, \quad i=1,2, \ldots, n .
$$

For any fixed root $\alpha$, the domain $D_{+}^{e}=D_{+} \cup r_{\alpha} D_{+}$(where each point is taken only once) can be taken as a closure of the fundamental domain of the even Weyl group $W_{e}$. The fundamental domain of $W_{e}$ is the set $D_{+}^{e}$ without its boundary. 
Let us describe a fundamental domain for the group $W^{\text {aff }}$. Since $W \subset W^{\text {aff }}$, it can be chosen as a subset of the dominant Weyl chamber for $W$.

We have seen that the element $r_{0} \in W^{\text {aff }}$ is a reflection with respect to the hyperplane $X_{n-1}+\xi^{\vee} / 2$, orthogonal to the root $\xi$ and containing the point $\xi^{\vee} / 2$. This hyperplane is given by the equation (2.21). This equation shows that the hyperplane $X_{n-1}+\xi^{\vee} / 2$ intersects the axes, determined by the vectors $\omega_{i}$, in the points $\omega_{i} / q_{i}, i=1,2, \ldots, n$, where $q_{i}$ are such as in (2.21). We create the simplex with $n+1$ vertices in the points

$$
0, \frac{\omega_{1}}{q_{1}}, \ldots, \frac{\omega_{n}}{q_{n}} .
$$

By (2.21), this simplex consists of all points $y$ of the dominant Weyl chamber for which $\langle y, \xi\rangle \leq 1$. Clearly, the interior $F$ of this simplex belongs to the dominant Weyl chamber. The following theorem is true (see, for example, [7]):

Theorem 1. The set $F$ is a fundamental domain for the affine Weyl group $W^{\text {aff }}$.

For the rank 2 cases the fundamental domain is the interior of the simplex with the following vertices:

$$
\begin{array}{ll}
A_{2}: & \left\{0, \omega_{1}, \omega_{2}\right\}, \\
C_{2}: & \left\{0, \omega_{1}, \omega_{2}\right\}, \\
G_{2}: & \left\{0, \frac{\omega_{1}}{2}, \omega_{2}\right\} .
\end{array}
$$

A fundamental domain of the even affine group $W_{e}^{\text {aff }}$ can be taken in such a way that it is contained in the fundamental domain $D_{+}^{e}$ of $W_{e}$. Namely, the set $\bar{F} \cup r_{\alpha} \bar{F}$ (where each point is taken only once) without its boundary satisfies this condition and is a $W_{e}^{\text {aff }}$-fundamental domain.

\section{$3 W_{e}$-orbits}

\subsection{Definition}

As we have seen, the $(n-1)$-dimensional linear subspaces $X_{\alpha}$ of $E_{n}$, orthogonal to positive roots $\alpha$ and containing the origin, divide the space $E_{n}$ into connected parts, which are called Weyl chambers. A number of such chambers is equal to an order of the corresponding Weyl group $W$. Elements of the Weyl group permute these chambers. A single chamber $D_{+}$such that $\left\langle\alpha_{i}, x\right\rangle \geq 0, x \in D_{+}, i=1,2, \ldots, n$, is the dominant Weyl chamber. We fix a root $\alpha$ and create a set $D_{+}^{e}=D_{+} \cup r_{\alpha} D_{+}$, where $r_{\alpha}$ is the reflection corresponding to the root $\alpha$. The sets, received from $D_{+}^{e}$ by action by elements of $W_{e}$ are called even Weyl chambers. Clearly, they depend on choosing of the root $\alpha$. However, different choices of $\alpha$ (and therefore, of even Weyl chambers) does not change a set of $W_{e}$-orbits, which are considered below.

The cone of dominant integral weights $P_{+}$belongs to the dominant Weyl chamber $D_{+}$. By $P_{+}^{e}$ we denote the set $P_{+}^{e} \cup r_{\alpha} P_{+}^{e}$ (where each point is taken only once). Then $P_{+}^{e} \subset D_{+}^{e}$.

Let $y$ be an arbitrary dominant element of the Euclidean space $E_{n}$. We act upon $y$ by all elements of the Weyl group $W$. As a result, we obtain the set $w y, w \in W / W_{y}$ (where $W_{y}$ is the subgroup of elements of $W$ leaving $y$ invariant), which is called a Weyl group orbit or a $W$-orbit of the point $y$. A $W$-orbit of a point $y \in D_{+}$is denoted by $O(y)$ or $O_{W}(y)$. A size of an orbit $O(y)$ is a number $|O(y)|$ of its elements. Each Weyl chamber contains only one point of a fixed orbit $Q(y)$.

Now we act upon element $y \in E_{n}$ by all elements of the even Weyl group $W_{e}$. As a result, we obtain a set of elements $w y, w \in W_{e}$ (each point is taken only once), which is called an even 
Weyl group orbit or a $W_{e}$-orbit of the point $y$. A $W_{e}$-orbit of a point $y \in D_{+}^{e}$ is denoted by $O_{e}(y)$ or $O_{W_{e}}(y)$. Each even Weyl chamber contains only one point of a fixed orbit $Q_{e}(y)$.

$W_{e}$-orbits $O_{e}(y)$ do not depend on a choice of a root $\alpha$ with respect to which we construct the even dominant Weyl chamber $D_{+}^{e}=D_{+} \cup r_{\alpha} D_{+}$.

There are two types of $W_{e}$-orbits: orbits $O_{e}(y)$ which contain a point of the dominant Weyl chamber $D_{+}$(we call them $W_{e^{-}}$orbits of the fist type) and orbits which do not contain such

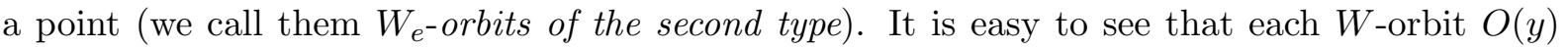
with strictly dominant $y$ consists of two $W_{e}$-orbits, one of them is of the first type and the second of the second type. Namely,

$$
O(y)=O_{e}(y) \bigcup O_{e}\left(r_{\alpha} y\right)
$$

where the $W_{e}$-orbit $O_{e}\left(r_{\alpha} y\right)$ does not depend on a choice of the root $\alpha$, that is, $O_{e}\left(r_{\alpha} y\right)$ are the same for all positive roots $\alpha$ of a given root system. Moreover, the orbit $O_{e}\left(r_{\alpha} y\right)$ is obtained from the orbit $O_{e}(y)$ by acting upon each point of $O_{e}(y)$ by reflection $r_{\alpha}, O_{e}\left(r_{\alpha} y\right)=r_{\alpha} O_{e}(y)$.

If $y$ belongs to some wall of the dominant Weyl chamber, then the $W_{e}$-orbit $O_{e}(y)$ coincides with the $W$-orbit $O(y)$.

Example. The case $A_{1}$. The Weyl group $W$ of $A_{1}$ consists of two elements 1 and $r_{\alpha}$, where $\alpha$ is a unique positive root of $A_{1}$. The element $r_{\alpha}$ is a reflection and, therefore, $\operatorname{det} r_{\alpha}=-1$. Thus, the subgroup $W_{e}$ in this case contains only one element 1 . This means that each point of the real line is a $W_{e}$-orbit for $A_{1}$. In particular, $O_{e}(y), y>0$, belongs to orbits, corresponding to dominant elements. The other orbits (except for the orbit $O_{e}(0)$ ) are obtained by acting by the reflection $r_{\alpha}$.

\section{$3.2 W_{e^{-o r b i t s}}$ of $A_{2}, C_{2}, G_{2}$}

In this subsection we give $W_{e}$-orbits for the rank two cases. Orbits will be given by coordinates in the $\omega$-basis. Points of the orbits will be denoted as $(a b)$, where $a$ and $b$ are $\omega$-coordinates.

If $a>0$ and $b>0$, then the $W_{e}$-orbits $O_{e}(a b)$ and $r_{\alpha} O(a b) \equiv O_{e}(-a a+b)$ of $A_{2}$ contain points

$$
\begin{aligned}
A_{2}: & O_{e}(a b) \ni(a b),(-a-b a),(b-a-b), \\
& O_{e}(-a a+b) \ni(-a a+b),(a+b-b),(-b-a) .
\end{aligned}
$$

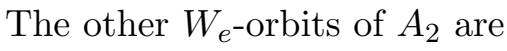

$$
\begin{aligned}
& A_{2}: \quad O_{e}(a 0) \ni(a 0),(-a a),(0-a), \\
& O_{e}\left(\begin{array}{lll}
0 & b
\end{array}\right) \ni\left(\begin{array}{ll}
0 & b
\end{array}\right),\left(\begin{array}{ll}
b & -b
\end{array}\right),\left(\begin{array}{ll}
-b & 0
\end{array}\right) \text {. }
\end{aligned}
$$

In the cases of $C_{2}$ and $G_{2}$ (where the second simple root is the longer one for $C_{2}$ and the shorter one for $G_{2}$ ) for $a>0$ and $b>0$ we have

$$
\begin{aligned}
C_{2}: & O_{e}(a b) \ni(a b),(a+2 b-a-b),(-a-b),(-a-2 b a+b), \\
& O_{e}(-a a+b) \ni(-a a+b),(a+2 b-b),(a-a-b),(-a-2 b b), \\
G_{2}: & O_{e}(a b) \ni \pm(a b), \pm(2 a+b-3 a-b), \pm(-a-b 3 a+2 b), \\
& O_{e}(-a 3 a+b) \ni \pm(-a 3 a+b), \pm(a+b-b), \pm(-2 a-b 3 a+2 b),
\end{aligned}
$$

where $\pm(c d)$ means two points $(c d)$ and $(-c-d)$.

We also give $W_{e^{-o r b i t s}}$ for which one of the numbers $a, b$ vanish:

$$
C_{2}: \quad O_{e}(a 0) \ni \pm(a 0), \pm(-a a),
$$




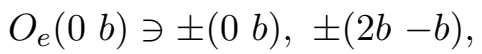

$$
\begin{aligned}
& G_{2}: \quad O_{e}(a 0) \ni \pm(a 0), \pm(-a 3 a), \pm(2 a-3 a),
\end{aligned}
$$

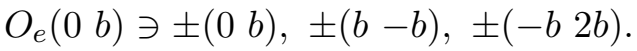

As we see, for each point $(c d)$ of an orbit $O_{e}$ of $C_{2}$ or $G_{2}$ there exists in the orbit the point $(-c-d)$.

\subsection{The case of $A_{n}$}

In the cases of Coxeter-Dynkin diagrams $A_{n-1}, B_{n}, C_{n}, D_{n}$, root and weight lattices, even Weyl groups and orbits are described in a simple way if to use the orthogonal coordinate system in $E_{n}$. In particular, this coordinate system is useful under practical work with orbits.

In the case $A_{n}$ it is convenient to describe root and weight lattices, even Weyl group and orbit functions in the subspace of the Euclidean space $E_{n+1}$, given by the equation

$$
x_{1}+x_{2}+\cdots+x_{n+1}=0
$$

where $x_{1}, x_{2}, \ldots, x_{n+1}$ are orthogonal coordinates of a point $x \in E_{n+1}$. The unit vectors in directions of these coordinates are denoted by $\mathbf{e}_{j}$, respectively. Clearly, $\mathbf{e}_{i} \perp \mathbf{e}_{j}, i \neq j$. The set of roots of $A_{n}$ is given by the vectors

$$
\alpha_{i j}=\mathbf{e}_{i}-\mathbf{e}_{j}, \quad i \neq j .
$$

The roots $\alpha_{i j}=\mathbf{e}_{i}-\mathbf{e}_{j}, i<j$, are positive and the roots

$$
\alpha_{i} \equiv \alpha_{i, i+1}=\mathbf{e}_{i}-\mathbf{e}_{i+1}, \quad i=1,2, \ldots, n,
$$

constitute the system of simple roots.

If $x=\sum_{i=1}^{n+1} x_{i} \mathbf{e}_{i}, x_{1}+x_{2}+\cdots+x_{n+1}=0$, is a point of $E_{n+1}$, then this point belongs to the dominant Weyl chamber $D_{+}$if and only if

$$
x_{1} \geq x_{2} \geq \cdots \geq x_{n+1} .
$$

Indeed, if this condition is fulfilled, then $\left\langle x, \alpha_{i}\right\rangle=x_{i}-x_{i+1} \geq 0, i=1,2, \ldots, n$, and $x$ is dominant. Conversely, if $x$ is dominant, then $\left\langle x, \alpha_{i}\right\rangle \geq 0$ and this condition is fulfilled. The point $x$ is strictly dominant if and only if

$$
x_{1}>x_{2}>\cdots>x_{n+1} .
$$

If $\alpha=\alpha_{12}$, the even dominant chamber $D_{+}^{e}=D_{+} \cup r_{\alpha} D_{+}$consists of points $x$ such that

$$
x_{1} \geq x_{2} \geq x_{3} \geq \cdots \geq x_{n+1} \quad \text { or } \quad x_{2} \geq x_{1} \geq x_{3} \geq \cdots \geq x_{n+1},
$$

that is, such that

$$
x_{2}, x_{2} \geq x_{3} \geq \cdots \geq x_{n+1}
$$

If $\lambda=\sum_{i=1}^{n} \lambda_{i} \omega_{i}$, then the $\omega$-coordinates $\lambda_{i}$ are connected with the orthogonal coordinates $x_{j}$ of $\lambda=\sum_{i=1}^{n+1} x_{i} \mathbf{e}_{i}$ by the formulas

$$
x_{1}=\frac{n}{n+1} \lambda_{1}+\frac{n-1}{n+1} \lambda_{2}+\frac{n-2}{n+1} \lambda_{3}+\cdots+\frac{2}{n+1} \lambda_{n-1}+\frac{1}{n+1} \lambda_{n},
$$




$$
\begin{aligned}
& x_{2}=-\frac{1}{n+1} \lambda_{1}+\frac{n-1}{n+1} \lambda_{2}+\frac{n-2}{n+1} \lambda_{3}+\cdots+\frac{2}{n+1} \lambda_{n-1}+\frac{1}{n+1} \lambda_{n} \text {, } \\
& x_{3}=-\frac{1}{n+1} \lambda_{1}-\frac{2}{n+1} \lambda_{2}+\frac{n-2}{n+1} \lambda_{3}+\cdots+\frac{2}{n+1} \lambda_{n-1}+\frac{1}{n+1} \lambda_{n}, \\
& x_{n}=-\frac{1}{n+1} \lambda_{1}-\frac{2}{n+1} \lambda_{2}-\frac{3}{n+1} \lambda_{3}-\cdots-\frac{n-1}{n+1} \lambda_{n-1}+\frac{1}{n+1} \lambda_{n}, \\
& x_{n+1}=-\frac{1}{n+1} \lambda_{1}-\frac{2}{n+1} \lambda_{2}-\frac{3}{n+1} \lambda_{3}-\cdots-\frac{n-1}{n+1} \lambda_{n-1}-\frac{n}{n+1} \lambda_{n} .
\end{aligned}
$$

The inverse formulas are

$$
\lambda_{i}=x_{i}-x_{i+1}, \quad i=1,2, \ldots, n .
$$

By means of the formula

$$
r_{\alpha} \lambda=\lambda-\frac{2\langle\lambda, \alpha\rangle}{\langle\alpha, \alpha\rangle} \alpha
$$

for the reflection with respect to the hyperplane, orthogonal to a root $\alpha$, we can find how elements of the Weyl group $W\left(A_{n}\right)$ act upon points $\lambda \in E_{n+1}$. We conclude that the Weyl group $W\left(A_{n}\right)$ consists of all permutations of the orthogonal coordinates $x_{1}, x_{2}, \ldots, x_{n+1}$ of a point $\lambda$, that is, $W\left(A_{n}\right)$ coincides with the symmetric group $S_{n+1}$. Even permutations of $W\left(A_{n}\right)$ constitute the even Weyl group $W_{e}\left(A_{n}\right)$. It is the alternating subgroup $S_{n+1}^{e}$ of the group $S_{n+1}$. This subgroup is simple. Transformations of $S_{n+1}^{e}$ are elements of the rotation group $S O(n+1)$.

Sometimes (for example, if we wish for coordinates to be integers or non-negative integers), it is convenient to introduce orthogonal coordinates $y_{1}, y_{2}, \ldots, y_{n+1}$ for $A_{n}$ in such a way that

$$
y_{1}+y_{2}+\cdots+y_{n+1}=m
$$

where $m$ is some fixed real number. They are obtained from the previous orthogonal coordinates by adding the same number $m /(n+1)$ to each coordinate. Then, as one can see from (3.13), $\omega$-coordinates $\lambda_{i}=y_{i}-y_{i+1}$ and the group $W$ and $W_{e}$ do not change. Sometimes, it is natural to use orthogonal coordinates $y_{1}, y_{2}, \ldots, y_{n+1}$ for which all $y_{i}$ are non-negative.

$W_{e}$-orbits $O_{e}(\lambda)$ for strictly dominant $\lambda$ can be constructed by means of signed $W$-orbits. Signed $W$-orbit $O^{ \pm}(\lambda)$ were introduced in [8]. The signed orbit $O^{ \pm}(\lambda), \lambda=\left(x_{1}, x_{2}, \ldots, x_{n+1}\right)$, $x_{1}>x_{2}>\cdots>x_{n+1}$, consists of all points

$$
\left(x_{i_{1}}, x_{i_{2}}, \ldots, x_{i_{n+1}}\right)^{\operatorname{sgn}(\operatorname{det} w)}
$$

obtained from $\left(x_{1}, x_{2}, \ldots, x_{n+1}\right)$ by permutations $w \in W \equiv S_{n+1}$ (instead of $\operatorname{sgn}(\operatorname{det} w)$ we sometimes write simply $\operatorname{det} w)$.

The signed $W$-orbit $O^{ \pm}(\lambda)$ splits into two $W_{e}$-orbits: one of them coincides with $O_{e}(\lambda)$ and the second with $O_{e}\left(r_{\alpha} \lambda\right)$, where $\alpha$ is a positive root of $A_{n}$. The $W_{e}$-orbit $O_{e}(\lambda)$ contains the points of the $W$-orbit $O^{ \pm}(\lambda)$, which have the sign plus and $O_{e}\left(r_{\alpha} \lambda\right)$ contains the points with the sign minus.

If $\lambda$ is dominant but not strictly dominant, then the $W_{e}$-orbit $O_{e}(\lambda)$ coincides with the $W$ orbit $O(\lambda)$. Description of $W$-orbits of $A_{n}$ in orthogonal coordinates see in [7, Subsection 3.1].

\subsection{The case of $B_{n}$}

Orthogonal coordinates of a point $x \in E_{n}$ are denoted by $x_{1}, x_{2}, \ldots, x_{n}$. We denote by $\mathbf{e}_{i}$ the corresponding unit vectors. Then the set of roots of $B_{n}$ is given by the vectors

$$
\alpha_{ \pm i, \pm j}= \pm \mathbf{e}_{i} \pm \mathbf{e}_{j}, \quad i \neq j, \quad \alpha_{ \pm i}= \pm \mathbf{e}_{i}, \quad i=1,2, \ldots, n
$$


(all combinations of signs must be taken). The roots $\alpha_{i, \pm j}=\mathbf{e}_{i} \pm \mathbf{e}_{j}, i<j, \alpha_{i}=\mathbf{e}_{i}, i=1,2, \ldots, n$, are positive and $n$ roots

$$
\alpha_{i}:=\mathbf{e}_{i}-\mathbf{e}_{i+1}, \quad i=1,2, \ldots, n-1, \quad \alpha_{n}=\mathbf{e}_{n}
$$

constitute the system of simple roots.

A point $\lambda=\sum_{i=1}^{n} x_{i} \mathbf{e}_{i} \in E_{n}$ belongs to the dominant Weyl chamber $D_{+}$if and only if

$$
x_{1} \geq x_{2} \geq \cdots \geq x_{n} \geq 0 .
$$

Moreover, this point is strictly dominant if and only if

$$
x_{1}>x_{2}>\cdots>x_{n}>0 .
$$

The even dominant chamber can be taken consisting of points $x$ such that

$$
x_{1} \geq x_{2} \geq x_{3} \geq \cdots \geq x_{n} \geq 0 \quad \text { or } \quad x_{2} \geq x_{1} \geq x_{2} \geq \cdots \geq x_{n} \geq 0,
$$

that is, such that

$$
x_{1}, x_{2} \geq x_{3} \geq \cdots \geq x_{n} \geq 0 .
$$

If $\lambda=\sum_{i=1}^{n} \lambda_{i} \omega_{i}$, then the $\omega$-coordinates $\lambda_{i}$ are connected with the orthogonal coordinates $x_{j}$ of $\lambda=\sum_{i=1}^{n} x_{i} \mathbf{e}_{i}$ by the formulas

$$
\begin{aligned}
& x_{1}=\lambda_{1}+\lambda_{2}+\cdots+\lambda_{n-1}+\frac{1}{2} \lambda_{n}, \\
& x_{2}=\lambda_{2}+\cdots+\lambda_{n-1}+\frac{1}{2} \lambda_{n}, \\
& \text {.......................... } \\
& x_{n}=\quad \frac{1}{2} \lambda_{n},
\end{aligned}
$$

The inverse formulas are

$$
\lambda_{i}=x_{i}-x_{i+1}, \quad i=1,2, \ldots, n-1, \quad \lambda_{n}=2 x_{n} .
$$

It is easy to see that if $\lambda \in P_{+}$, then the coordinates $x_{1}, x_{1}, \ldots, x_{n}$ are all integers or all halfintegers.

The Weyl group $W\left(B_{n}\right)$ of $B_{n}$ consists of all permutations of the orthogonal coordinates $x_{1}, x_{2}, \ldots, x_{n}$ of a point $\lambda$ with possible sign alternations of any number of them. Moreover, $\operatorname{det} w$ is equal to \pm 1 depending on whether $w$ is a product of even or odd number of reflections and alternations of signs. A sign of $\operatorname{det} w$ can be determined as follows. We represent $w$ as a product $w=\epsilon s$, where $s$ is a permutation of $\left(x_{1}, x_{2}, \ldots, x_{n}\right)$ and $\epsilon$ is an alternation of signs of coordinates. Then $\operatorname{det} w=(\operatorname{det} s) \epsilon_{i_{1}} \epsilon_{i_{2}} \cdots \epsilon_{i_{n}}$, where $\operatorname{det} s$ is defined as in the previous subsection and $\epsilon_{i_{j}}=-1$ in the case of change of a sign of $i_{j}$-th coordinate and $\epsilon_{i_{j}}=1$ otherwise. This show haw to determine the even Weyl group $W_{e}\left(B_{n}\right)$ as a subgroup of $W\left(B_{n}\right)$.

$W_{e}\left(B_{n}\right)$-orbits $O_{e}(\lambda)$ with strictly dominant $\lambda$ can be constructed by means of signed orbits $O^{ \pm}(\lambda)$. The signed orbit $O^{ \pm}(\lambda), \lambda=\left(x_{1}, x_{2}, \ldots, x_{n}\right), x_{1}>x_{2}>\cdots>x_{n}>0$, consists of all points

$$
\left( \pm x_{i_{1}}, \pm x_{i_{2}}, \ldots, \pm x_{i_{n}}\right)^{\operatorname{det} w}
$$

(each combination of signs is possible) obtained from $\left(x_{1}, x_{2}, \ldots, x_{n}\right)$ by permutations and alternations of signs which constitute an element $w$ of the Weyl group $W\left(B_{n}\right)$. The signed orbit $O^{ \pm}(\lambda)$ splits into two $W_{e}$-orbits: one of them $O_{e}(\lambda)$ consists of all points of $O^{ \pm}(\lambda)$, which have the sign plus, and the second one consists of all points with the sign minus.

If a dominant element $\lambda$ is not strictly dominant, then the $W_{e^{-}}$orbit $O_{e}(\lambda)$ coincides with the $W$-orbit $O(\lambda)$. 


\subsection{The case of $C_{n}$}

In the orthogonal coordinate system of the Euclidean space $E_{n}$ the set of roots of $C_{n}$ is given by the vectors

$$
\alpha_{ \pm i, \pm j}= \pm \mathbf{e}_{i} \pm \mathbf{e}_{j}, \quad i \neq j, \quad \alpha_{ \pm i}= \pm 2 \mathbf{e}_{i}, \quad i=1,2, \ldots, n,
$$

where $\mathbf{e}_{i}$ is the unit vector in the direction of $i$-th coordinate $x_{i}$ (all combinations of signs must be taken). The roots $\alpha_{i, \pm j}=\mathbf{e}_{i} \pm \mathbf{e}_{j}, i<j$, and $\alpha_{i}=2 \mathbf{e}_{i}, i=1,2, \ldots, n$, are positive and $n$ roots

$$
\alpha_{i}:=\mathbf{e}_{i}-\mathbf{e}_{i+1}, \quad i=1,2, \ldots, n-1, \quad \alpha_{n}=2 \mathbf{e}_{n}
$$

constitute the system of simple roots.

A point $\lambda=\sum_{i=1}^{n} x_{i} \mathbf{e}_{i} \in E_{n}$ belongs to the dominant Weyl chamber $D_{+}$if and only if

$$
x_{1} \geq x_{2} \geq \cdots \geq x_{n} \geq 0 .
$$

This point is strictly dominant if and only if

$$
x_{1}>x_{2}>\cdots>x_{n}>0 .
$$

The even dominant chamber can be taken consisting of points $x$ such that

$$
x_{1} \geq x_{2} \geq x_{3} \geq \cdots \geq x_{n} \geq 0 \quad \text { or } \quad x_{2} \geq x_{1} \geq x_{3} \geq \cdots \geq x_{n} \geq 0,
$$

that is, such that

$$
x_{1}, x_{2} \geq x_{3} \geq \cdots \geq x_{n} \geq 0 .
$$

If $\lambda=\sum_{i=1}^{n} \lambda_{i} \omega_{i}$, then the $\omega$-coordinates $\lambda_{i}$ are connected with the orthogonal coordinates $x_{j}$ of $\lambda=\sum_{i=1}^{n} x_{i} \mathbf{e}_{i}$ by the formulas

$$
\begin{aligned}
& x_{1}=\lambda_{1}+\lambda_{2}+\cdots+\lambda_{n-1}+\lambda_{n}, \\
& x_{2}=\quad \lambda_{2}+\cdots+\lambda_{n-1}+\lambda_{n}, \\
& \cdots \ldots \ldots+\cdots+\cdots+\cdots+\lambda_{n} .
\end{aligned}
$$

The inverse formulas are

$$
\lambda_{i}=x_{i}-x_{i+1}, \quad i=1,2, \ldots, n-1, \quad \lambda_{n}=x_{n} .
$$

If $\lambda \in P_{+}$, then all coordinates $x_{i}$ are integers.

The Weyl group $W\left(C_{n}\right)$ of $C_{n}$ consists of all permutations of the orthogonal coordinates $x_{1}, x_{2}, \ldots, x_{n}$ of a point $\lambda$ with sign alternations of some of them, that is, this Weyl group acts on orthogonal coordinates exactly in the same way as the Weyl group $W\left(B_{n}\right)$ does. Moreover, $\operatorname{det} w$ is equal to \pm 1 depending on whether $w$ consists of even or odd numbers of reflections and alternations of signs. Since $W\left(C_{n}\right)=W\left(B_{n}\right)$, then a sign of $\operatorname{det} w$ is determined as in the case $B_{n}$.

The signed orbit $O^{ \pm}(\lambda), \lambda=\left(x_{1}, x_{2}, \ldots, x_{n}\right), x_{1}>x_{2}>\cdots>x_{n}>0$, consists of all points

$$
\left( \pm x_{i_{1}}, \pm x_{i_{2}}, \ldots, \pm x_{i_{n+1}}\right)^{\operatorname{det} w}
$$


(each combination of signs is possible) obtained from $\left(x_{1}, x_{2}, \ldots, x_{n}\right)$ by permutations and alternations of signs which constitute an element $w$ of the Weyl group $W\left(C_{n}\right)$, that is, in the orthogonal coordinates signed orbits for $C_{n}$ coincide with signed orbits of $B_{n}$. This determine how to separate the subgroup $W_{e}\left(C_{n}\right)$ in the group $W\left(C_{n}\right)$.

The signed orbit $O^{ \pm}(\lambda), \lambda=\left(x_{1}, x_{2}, \ldots, x_{n}\right), x_{1}>x_{2}>\cdots>x_{n}>0$, splits into two $W_{e^{-}}$ orbits: one of them $O_{e}(\lambda)$ consists of all points of $O^{ \pm}(\lambda)$, which have the sign plus, and the second one consists of all points with the sign minus.

If a dominant element $\lambda$ is not strictly dominant, then the $W_{e}$-orbit $O_{e}(\lambda)$ coincides with the $W$-orbit $O(\lambda)$.

\subsection{The case of $D_{n}$}

In the orthogonal coordinate system of the Euclidean space $E_{n}$ the set of roots of $D_{n}$ is given by the vectors

$$
\alpha_{ \pm i, \pm j}= \pm \mathbf{e}_{i} \pm \mathbf{e}_{j}, \quad i \neq j,
$$

where $\mathbf{e}_{i}$ is the unit vector in the direction of $i$-th coordinate (all combinations of signs must be taken). The roots $\alpha_{i, \pm j}=\mathbf{e}_{i} \pm \mathbf{e}_{j}, i<j$, are positive and $n$ roots

$$
\alpha_{i}:=\mathbf{e}_{i}-\mathbf{e}_{i+1}, \quad i=1,2, \ldots, n-1, \quad \alpha_{n}=\mathbf{e}_{n-1}+\mathbf{e}_{n}
$$

constitute the system of simple roots.

$$
\begin{aligned}
& \text { If } \lambda=\sum_{i=1}^{n} x_{i} \mathbf{e}_{i} \text {, then this point belongs to the dominant Weyl chamber } D_{+} \text {if and only if } \\
& \quad x_{1} \geq x_{2} \geq \cdots \geq x_{n-1} \geq\left|x_{n}\right| .
\end{aligned}
$$

This point is strictly dominant if and only if

$$
x_{1}>x_{2}>\cdots>x_{n-1}>\left|x_{n}\right|
$$

(in particular, $x_{n}$ can take the value 0 ).

The even dominant chamber can be taken consisting of points $x$ such that

$$
x_{1} \geq x_{2} \geq x_{3} \geq \cdots \geq\left|x_{n}\right| \quad \text { or } \quad x_{2} \geq x_{1} \geq x_{3} \geq \cdots \geq\left|x_{n}\right|,
$$

that is, such that

$$
x_{1}, x_{2} \geq x_{3} \geq \cdots \geq x_{n-1} \geq\left|x_{n}\right| .
$$

If $\lambda=\sum_{i=1}^{n} \lambda_{i} \omega_{i}$, then the $\omega$-coordinates $\lambda_{i}$ are connected with the orthogonal coordinates $x_{j}$ of $\lambda=\sum_{i=1}^{n} x_{i} \mathbf{e}_{i}$ by the formulas

$$
\begin{aligned}
& x_{1}=\lambda_{1}+\lambda_{2}+\cdots+\lambda_{n-2}+\frac{1}{2}\left(\lambda_{n-1}+\lambda_{n}\right) \text {, } \\
& x_{2}=\lambda_{2}+\cdots+\lambda_{n-2}+\frac{1}{2}\left(\lambda_{n-1}+\lambda_{n}\right) \text {, } \\
& \text {.............................. } \\
& x_{n-1}= \\
& \frac{1}{2}\left(\lambda_{n-1}+\lambda_{n}\right), \\
& x_{n}= \\
& \frac{1}{2}\left(\lambda_{n-1}-\lambda_{n}\right) \text {, }
\end{aligned}
$$


The inverse formulas are

$$
\lambda_{i}=x_{i}-x_{i+1}, \quad i=1,2, \ldots, n-2, \quad \lambda_{n-1}=x_{n-1}+x_{n}, \quad \lambda_{n}=x_{n-1}-x_{n} .
$$

If $\lambda \in P_{+}$, then the coordinates $x_{1}, x_{2}, \ldots, x_{n}$ are all integers or all half-integers.

The Weyl group $W\left(D_{n}\right)$ of $D_{n}$ consists of all permutations of the orthogonal coordinates $x_{1}, x_{2}, \ldots, x_{n}$ of a point $\lambda$ with sign alternations of even number of them. Moreover, $\operatorname{det} w$ is equal to \pm 1 and a sign of $\operatorname{det} w$ is determined as follows. The element $w \in W\left(D_{n}\right)$ can be represented as a product $w=\tau s$, where $s$ is a permutation from $S_{n}$ and $\tau$ is an alternation of even number of coordinates. Then $\operatorname{det} w=\operatorname{det} s$. Indeed, since an alternation of signs of two coordinates $x_{i}$ and $x_{j}$ is a product of two reflections $r_{\alpha}$ with $\alpha=\mathbf{e}_{i}+\mathbf{e}_{j}$ and with $\alpha=\mathbf{e}_{i}-\mathbf{e}_{j}$, a sign of the determinant of this alternation is plus. (Note that $\left|W\left(D_{n}\right)\right|=\frac{1}{2}\left|W\left(B_{n}\right)\right|$.)

Now we may state that the even Weyl group $W_{e}\left(D_{n}\right)$ consists of products $\tau s$, where $s$ runs over even permutations of $S_{n}$ and $\tau$ runs over alternations of even numbers of coordinates.

The signed orbit $O^{ \pm}(\lambda), \lambda=\left(x_{1}, x_{2}, \ldots, x_{n}\right), x_{1}>x_{2}>\cdots>x_{n}>0$, for $D_{n}$ consists of all points

$$
\left( \pm x_{i_{1}}, \pm x_{i_{2}}, \ldots, \pm x_{i_{n}}\right)^{\operatorname{det} w}
$$

obtained from $\left(x_{1}, x_{2}, \ldots, x_{n}\right)$ by permutations and alternations of even number of signs which constitute an element $w$ of the Weyl group $W\left(D_{n}\right)$. This signed orbit splits into two $W_{e^{-}}$orbits: one of them $O_{e}(\lambda)$ consists of all points of $O^{ \pm}(\lambda)$, which have the sign plus, and the second one consists of all points with the sign minus.

If a dominant element $\lambda$ is not strictly dominant, then the $W_{e}\left(D_{n}\right)$-orbit $O_{e}(\lambda)$ coincides with the $W\left(D_{n}\right)$-orbit $O(\lambda)$.

\subsection{Fundamental domains of $W_{e}^{\text {aff }}$}

Using the explicit formula for the antisymmetric orbit function $\varphi_{\rho}(x)$, where $\rho$ is a half of positive roots, we have derived in [8] explicit forms of the fundamental domains of $W^{\text {aff }}$ for the cases $A_{n}$, $B_{n}, C_{n}, D_{n}$. They easily determine fundamental domains $F_{e}$ for the corresponding even affine Weyl groups $W_{e}^{\text {aff }}$.

(a) The fundamental domain $F_{e}\left(A_{n}\right)$ of the even affine Weyl group $W_{e}^{\text {aff }}\left(A_{n}\right)$ is contained in the domain of real points $x=\left(x_{1}, x_{2}, \ldots, x_{n+1}\right)$ such that

$$
x_{1}, x_{2}>x_{3}>\cdots>x_{n+1}, \quad x_{1}+x_{2}+\cdots+x_{n+1}=0 .
$$

Moreover, a point $x$ of this domain belongs to $F_{e}\left(A_{n}\right)$ if and only if $x_{1}+\left|x_{n+1}\right|<1$ and $x_{1}>x_{2}$, or $x_{2}+\left|x_{n+1}\right|<1$ and $x_{2}>x_{1}$.

(b) The fundamental domain $F_{e}\left(B_{n}\right)$ of $W_{e}^{\text {aff }}\left(B_{n}\right)$ is contained in the domain of points $x=$ $\left(x_{1}, x_{2}, \ldots, x_{n}\right)$ such that

$$
1>x_{1}, x_{2}>x_{3}>\cdots>x_{n}>0 .
$$

Moreover, a point $x$ of this domain belongs to $F_{e}\left(B_{n}\right)$ if and only if $x_{1}+x_{2}<1$.

(c) The fundamental domain $F_{e}\left(C_{n}\right)$ of $W_{e}^{\text {aff }}\left(C_{n}\right)$ consists of all points $x=\left(x_{1}, x_{2}, \ldots, x_{n}\right)$ such that

$$
\frac{1}{2}>x_{1}, x_{2}>x_{3}>\cdots>x_{n}>0 .
$$

(d) The fundamental domain $F_{e}\left(D_{n}\right)$ of $W_{e}^{\text {aff }}\left(D_{n}\right)$ is contained in the domain of points $x=$ $\left(x_{1}, x_{2}, \ldots, x_{n}\right)$ such that

$$
1>x_{1}, x_{2}>x_{3}>\cdots>x_{n-1}>\left|x_{n}\right| .
$$

Moreover, a point $x$ of this domain belongs to $F_{e}\left(D_{n}\right)$ if and only if $x_{1}+x_{2}<1$. 


\section{$3.8 W_{e^{-o r b i t s} \text { of } A_{3}}$}

$W_{e}$-orbits for $A_{3}, B_{3}$ and $C_{3}$ can be calculated by using the orthogonal coordinates on the corresponding Euclidean space, described above, and the description of action of the Weyl groups $W_{e}\left(A_{3}\right), W_{e}\left(B_{3}\right)$ and $W_{e}\left(C_{3}\right)$ in the orthogonal coordinate systems. Below we give results of such calculations. Points $\lambda$ of $W_{e}$-orbits are given in the $\omega$-coordinates in the form $(a b c)$, where $\lambda=a \omega_{1}+b \omega_{2}+c \omega_{3}$.

If $a>0, b>0, c>0$, then $W_{e}$-orbits $O_{e}(a b c)$ and $O_{e}(a+b-b b+c) \equiv r_{\alpha} O_{e}(a b c)$ of $A_{3}$ contain the points

$$
\begin{aligned}
& O_{e}(a b c) \ni(a b c),(a+b c-b-c),(a+b+c-b-c b), \\
& (-a a+b+c-c),(b-a-b a+b+c),(-a-b a b+c), \\
& O_{e}(a+b-b b+c) \ni(a+b-b b+c),(a b+c-c),(a+b+c-c-b) \text {, } \\
& (-a a+b c),(b+c-a-b-c a+b),(-b-a a+b+c)
\end{aligned}
$$

and the points, contragredient to these points, where the contragredient of the point $\left(a^{\prime} b^{\prime} c^{\prime}\right)$ is $\left(-c^{\prime}-b^{\prime}-a^{\prime}\right)$.

There exist also the $W_{e^{-o r b i t s}}$

$$
\begin{aligned}
& O_{e}\left(\begin{array}{lll}
a & b & 0
\end{array}\right) \ni\left(\begin{array}{lll}
a & b & 0
\end{array}\right),(a+b-b \quad b),(a+b \quad 0-b),(-a a+b 0),(-a-b a b), \\
& (b-a-b a+b) \text { and contragredient points; } \\
& O_{e}\left(\begin{array}{lll}
a & 0 & c
\end{array}\right) \ni\left(\begin{array}{lll}
a & 0 & c
\end{array}\right),(a c-c),(a+c-c 0),(-a a c),(0-a a+c), \\
& (-a a+c-c) \text { and contragredient points; } \\
& O_{e}\left(\begin{array}{lll}
0 & b & c
\end{array}\right) \ni\left(\begin{array}{lll}
0 & b & c
\end{array}\right),(b-b b+c),(0 b+c-c),(b+c-b-c b),(-b \quad 0 b+c) \text {, } \\
& (b c-b-c) \text { and contragredient points; } \\
& O_{e}\left(\begin{array}{lll}
a & 0 & 0
\end{array}\right) \ni\left(\begin{array}{lll}
a & 0 & 0
\end{array}\right),\left(\begin{array}{lll}
-a & a & 0
\end{array}\right),\left(\begin{array}{lll}
0 & 0 & -a
\end{array}\right),\left(\begin{array}{lll}
0 & -a & a
\end{array}\right) \text {; } \\
& O_{e}\left(\begin{array}{lll}
0 & b & 0
\end{array}\right) \ni\left(\begin{array}{lll}
0 & b & 0
\end{array}\right),\left(\begin{array}{lll}
b & -b & b
\end{array}\right),\left(\begin{array}{lll}
b & 0 & -b
\end{array}\right),\left(\begin{array}{lll}
-b & b & -b
\end{array}\right),\left(\begin{array}{lll}
0 & -b & 0
\end{array}\right),\left(\begin{array}{lll}
-b & 0 & b
\end{array}\right) \text {; } \\
& O_{e}\left(\begin{array}{lll}
0 & 0 & c
\end{array}\right) \ni\left(\begin{array}{lll}
0 & 0 & c
\end{array}\right),\left(\begin{array}{lll}
0 & c & -c
\end{array}\right),\left(\begin{array}{cll}
c & -c & 0
\end{array}\right),\left(\begin{array}{lll}
-c & 0 & 0
\end{array}\right) \text {. }
\end{aligned}
$$

\section{$3.9 W_{e}$-orbits of $B_{3}$}

As in the previous case, points $\lambda$ of $W_{e}$-orbits are given by the $\omega$-coordinates $(a b c)$, where $\lambda=a \omega_{1}+b \omega_{2}+c \omega_{3}$. The $W_{e}$-orbits $O_{e}(a b c)$ and $O_{e}(a+b-b 2 b+c) \equiv r_{\alpha} O_{e}(a b c), a>0$, $b>0, c>0$, of $B_{3}$ contain the points

$$
\begin{aligned}
O_{e}(a b c) & \ni(a b c),(b-a-b 2 a+2 b+c),(-a-b a 2 b+c),(a+b+c-b-c 2 b+c), \\
& (-a a+b+c-c),(-b-c-a 2 a+2 b+c),(-a-b-c-b 2 b+c), \\
& (a+2 b+c-a-b-c c),(-b a+2 b+c-2 a-2 b-c),(-a-2 b-c b+c-c), \\
& (b+c a+b-2 a-2 b-c),(a+b-a-2 b-c 2 b+c), \\
O_{e}(a+b- & b 2 b+c) \ni(a+b-b 2 b+c),(-a a+b c),(-b-a 2 a+2 b+c),(a b+c-c), \\
& (b+c-a-b-c 2 a+2 b+c),(-a-b-c a 2 b+c),(-a-2 b-c b c), \\
& (b a+b+c-2 a-2 b-c),(a+b+c-a-2 b-c 2 b+c),(-a-b-b-c 2 b+c), \\
& (a+2 b+c-a-b-c),(-b-c a+2 b+c-2 a-2 b-c)
\end{aligned}
$$

and also all these points taken with opposite signs of coordinates.

In the case $B_{3}$ there exist also the $W_{e}$-orbits $O_{e}(a b 0), O_{e}(a \quad 0 c), O_{e}\left(\begin{array}{ll}0 & b \\ c\end{array}\right)$, which are of the form

$$
O_{e}\left(\begin{array}{lll}
a & b & 0
\end{array}\right) \ni \pm(a b 0), \pm(a+b-b 2 b), \pm(-a a+b 0), \pm(b-a-b 2 a+2 b),
$$




$$
\begin{aligned}
& \pm(-a-b a 2 b), \pm(-b-a 2 a+2 b), \pm(-a-2 b b 0), \pm(-a-b-b 2 b), \\
& \pm(a+2 b-a-b 0), \pm(b a+b-2 a-2 b), \pm(a+b-a-2 b 2 b), \pm(-b a+2 b-2 a-2 b) \text {; } \\
& O_{e}\left(\begin{array}{lll}
a & 0 & c
\end{array}\right) \ni \pm\left(\begin{array}{lll}
a & 0 & c
\end{array}\right), \pm(-a a c), \pm(0-a 2 a+c), \pm(a c-c), \\
& \pm(a+c-c c), \pm(-a a+c-c), \pm(c-a-c 2 a+c), \pm(-a-c a c) \text {, } \\
& \pm(-c-a 2 a+c), \pm(-a-c 0 c), \pm(a+c-a-c c), \pm(0 a+c-2 a-c) \text {; } \\
& O_{e}\left(\begin{array}{lll}
0 & b & c
\end{array}\right) \ni \pm\left(\begin{array}{lll}
0 & b & c
\end{array}\right), \pm(b-b 2 b+c), \pm(-b 02 b+c), \pm\left(\begin{array}{ll}
0 & b+c-c
\end{array}\right) \text {, } \\
& \pm(b+c-b-c 2 b+c), \pm(-b-c 02 b+c), \pm(-2 b-c b c), \pm(-b-c-b 2 b+c) \text {, } \\
& \pm(2 b+c-b-c c), \pm(b b+c-2 b-c), \pm(b+c-2 b-c 2 b+c), \pm(-b 2 b+c-2 b-c) \text {. }
\end{aligned}
$$

The $W_{e}$-orbits $O_{e}\left(\begin{array}{lll}a & 0 & 0\end{array}\right), O_{e}\left(\begin{array}{lll}0 & b & 0\end{array}\right)$ and $O_{e}\left(\begin{array}{lll}0 & 0 & c\end{array}\right)$ consist of the points

$$
\begin{aligned}
& O_{e}\left(\begin{array}{lll}
a & 0 & 0
\end{array}\right) \ni \pm\left(\begin{array}{lll}
a & 0 & 0
\end{array}\right), \pm(a-a \quad 0), \pm\left(\begin{array}{lll}
0 & a & -2 a
\end{array}\right) \text {; } \\
& O_{e}\left(\begin{array}{lll}
0 & b & 0
\end{array}\right) \ni \pm\left(\begin{array}{lll}
0 & b & 0
\end{array}\right), \pm\left(\begin{array}{lll}
b & -b & 2 b
\end{array}\right), \pm\left(\begin{array}{lll}
-b & 0 & 2 b
\end{array}\right), \pm\left(\begin{array}{lll}
-2 b & b & 0
\end{array}\right), \pm(-b-b 2 b), \pm\left(\begin{array}{lll}
b & -2 b & 2 b
\end{array}\right) \text {; } \\
& O_{e}\left(\begin{array}{lll}
0 & 0 & c
\end{array}\right) \ni \pm\left(\begin{array}{lll}
0 & 0 & c
\end{array}\right), \pm(c-c c), \pm\left(\begin{array}{lll}
0 & c-c
\end{array}\right), \pm(-c \quad 0 c) \text {. }
\end{aligned}
$$

\section{$3.10 W_{e}$-orbits of $C_{3}$}

As in the previous cases, points $\lambda$ of $W_{e}$-orbits are given by the $\omega$-coordinates $(a b c)$, where $\lambda=a \omega_{1}+b \omega_{2}+c \omega_{3}$. The $W_{e}$ orbits $O_{e}(a b c)$ and $O_{e}(a+b-b b+c) \equiv r_{\alpha} O_{e}(a b c), a>0, b>0$, $c>0$, of $C_{3}$ contain the points

$$
\begin{aligned}
O_{e}(a b c) & \ni(a b c),(b-a-b a+b+c),(-a-b a b+c),(a+b+2 c-b-2 c b+c), \\
& (-a a+b+2 c-c),(-b-2 c-a a+b+c),(-a-b-2 c-b b+c), \\
& (a+2 b+2 c-a-b-2 c c),(-b a+2 b+2 c-a-b-c),(-a-2 b-2 c b+2 c-c), \\
& (b+2 c a+b-a-b-c),(a+b-a-2 b-2 c b+c), \\
O_{e}(a+b- & b b+c) \ni(a+b-b b+c),(-a a+b c),(-b-a a+b+c),(a b+2 c-c), \\
& (b+2 c-a-b-2 c a+b+c),(-a-b-2 c a b+c),(-a-2 b-2 c b c), \\
& (b a+b+2 c-a-b-c),(a+b+2 c-a-2 b-2 c b+c),(-a-b-b-2 c b+c), \\
& (a+2 b+2 c-a-b-c),(-b-2 c a+2 b+2 c-a-b-c)
\end{aligned}
$$

and also all these points taken with opposite signs of coordinates.

For the $W_{e}$-orbits $O_{e}\left(\begin{array}{lll}a & b & 0\end{array}\right), O_{e}(a \quad 0 c)$ and $O_{e}\left(\begin{array}{llc}0 & b\end{array}\right)$ we have

$$
\begin{aligned}
& O_{e}\left(\begin{array}{lll}
a & b & 0
\end{array}\right) \ni \pm\left(\begin{array}{lll}
a & b & 0
\end{array}\right), \pm(a+b-b \quad b), \pm(-a a+b 0), \pm(b-a-b a+b), \\
& \pm(-a-b a b), \pm(-b-a a+b), \pm(-a-2 b b 0), \pm(-a-b-b b), \\
& \pm(a+2 b-a-b 0), \pm(b a+b-a-b), \pm(a+b-a-2 b b), \pm(-b a+2 b-a-b) ; \\
& O_{e}\left(\begin{array}{lll}
a & 0 & c
\end{array}\right) \ni \pm\left(\begin{array}{lll}
a & 0 & c
\end{array}\right), \pm(-a a c), \pm(0-a a+c), \pm(a 2 c-c), \\
& \pm(a+2 c-2 c c), \pm(a+2 c-a-2 c c), \pm(0 a+2 c-a-c), \pm(-a a+2 c-c), \\
& \pm(2 c-a-2 c a+c), \pm(-a-2 c a c), \pm(-2 c-a a+c), \pm(-a-2 c 0 c) \text {; } \\
& O_{e}\left(\begin{array}{lll}
0 & b & c
\end{array}\right) \ni \pm\left(\begin{array}{lll}
0 & b & c
\end{array}\right), \pm(b-b b+c), \pm(-b \quad 0 b+c), \pm\left(\begin{array}{ll}
0 & b+2 c
\end{array}-c\right) \text {, } \\
& \pm(b+2 c-b-2 c b+c), \pm(-b-2 c 0 b+c), \pm(-2 b-2 c b c), \pm(-b-2 c-b b+c) \text {, } \\
& \pm(2 b+2 c-b-2 c c), \pm(b b+2 c-b-c), \pm(b+2 c-2 b-2 c b+c), \pm(-b 2 b+2 c-b-c) \text {. }
\end{aligned}
$$

The $W_{e}$-orbits $O_{e}\left(\begin{array}{lll}a & 0 & 0\end{array}\right), O_{e}\left(\begin{array}{lll}0 & b & 0\end{array}\right)$ and $O_{e}\left(\begin{array}{lll}0 & 0 & c\end{array}\right)$ consist of the points

$$
\begin{aligned}
& O_{e}\left(\begin{array}{lll}
a & 0 & 0
\end{array}\right) \ni \pm\left(\begin{array}{lll}
a & 0 & 0
\end{array}\right), \pm(a-a r), \pm\left(\begin{array}{lll}
0 & a & -a
\end{array}\right) \text {; } \\
& O_{e}\left(\begin{array}{lll}
0 & b & 0
\end{array}\right) \ni \pm\left(\begin{array}{lll}
0 & b & 0
\end{array}\right), \pm\left(\begin{array}{lll}
b-b & b
\end{array}\right), \pm(b 0-b), \pm(2 b-b 0), \pm(-b-b b), \pm(b-2 b b) \text {; } \\
& O_{e}\left(\begin{array}{lll}
0 & 0 & c
\end{array}\right) \ni \pm\left(\begin{array}{lll}
0 & 0 & c
\end{array}\right), \pm\left(\begin{array}{lll}
0 & 2 c & -c
\end{array}\right), \pm(2 c-2 c c), \pm(2 c 0-c) \text {. }
\end{aligned}
$$




\section{$4 \quad E$-orbit functions}

\subsection{Definition}

$E$-orbit functions are obtained from the exponential functions $e^{2 \pi \mathrm{i}\langle\lambda, x\rangle}, x \in E_{n}$, with fixed $\lambda=\left(\lambda_{1}, \lambda_{2}, \ldots, \lambda_{n}\right)$ by the procedure of symmetrization by means of the even Weyl group $W_{e}$. $E$-orbit functions are closely related to symmetric and antisymmetric $W$-orbit functions. For this reason, we first define the last functions.

Let $W$ be a Weyl group of transformations of the Euclidean space $E_{n}$. To each element $\lambda \in E_{n}$ from the dominant Weyl chamber (that is, $\left\langle\lambda, \alpha_{i}\right\rangle \geq 0$ for all simple roots $\alpha_{i}$ ) there corresponds a symmetric orbit function $\phi_{\lambda}$ on $E_{n}$, which is given by the formula

$$
\phi_{\lambda}(x)=\sum_{\mu \in O(\lambda)} e^{2 \pi \mathrm{i}\langle\mu, x\rangle}, \quad x \in E_{n}
$$

where $O(\lambda)$ is the $W$-orbit of the element $\lambda$. The number of summands is equal to the size $|O(\lambda)|$ of the orbit $O(\lambda)$ and we have $\phi_{\lambda}(0)=|O(\lambda)|$. Sometimes (see, for example, [4] and [5]), it is convenient to use a modified definition of orbit functions:

$$
\hat{\phi}_{\lambda}(x)=\left|W_{\lambda}\right| \phi_{\lambda}(x)
$$

where $W_{\lambda}$ is a subgroup in $W$ whose elements leave $\lambda$ fixed. Then for all orbit functions $\hat{\phi}_{\lambda}$ we have $\hat{\phi}_{\lambda}(0)=|W|$. The functions $\hat{\phi}_{\lambda}(x)$ can be defined as

$$
\hat{\phi}_{\lambda}(x)=\sum_{w \in W} e^{2 \pi \mathrm{i}\langle\mu, x\rangle} .
$$

Antisymmetric orbit functions are defined (see [2] and [6]) for dominant elements $\lambda$, which do not belong to a wall of the dominant Weyl chamber (that is, for strictly dominant elements $\lambda$ ). The antisymmetric orbit function, corresponding to such an element, is defined as

$$
\varphi_{\lambda}(x)=\sum_{w \in W}(\operatorname{det} w) e^{2 \pi \mathrm{i}\langle w \lambda, x\rangle}, \quad x \in E_{n} .
$$

A number of summands in (4.3) is equal to the size $|W|$ of the Weyl group $W$. We have $\varphi_{\lambda}(0)=0$.

$E$-orbit functions are defined for each element $\lambda$ of the domain $D_{+}^{e}=D_{+} \cup r_{\alpha} D_{+}$, where $D_{+}$ is the set of dominant elements of $E_{n}$ and $\alpha$ is a root of the root system (we assume that each point is taken only once). The $E$-orbit function $E_{\lambda}(x), \lambda \in D_{+}^{e}$, is given by the formula

$$
E_{\lambda}(x)=\sum_{\mu \in O_{e}(\lambda)} e^{2 \pi \mathrm{i}\langle\mu, x\rangle}, \quad x \in E_{n}
$$

where $O_{e}(\lambda)$ is the $W_{e}$-orbit of the point $\lambda$.

Sometimes, it is convenient to use normalized $E$-orbit functions defined as

$$
\hat{E}_{\lambda}(x)=\sum_{w \in W_{e}} e^{2 \pi \mathrm{i}\langle w \lambda, x\rangle}
$$

We have $\hat{E}_{\lambda}(x)=\left|W_{e}^{\lambda}\right| E_{\lambda}(x)$, where $W_{e}^{\lambda}$ is a subgroup of $W_{e}$ whose elements leave $\lambda$ invariant.

Example. E-orbit functions for $A_{1}$. In this case, there exists only one simple (positive) root $\alpha$. We have $\langle\alpha, \alpha\rangle=2$. Then the relation $2\langle\omega, \alpha\rangle /\langle\alpha, \alpha\rangle=1$ means that $\langle\omega, \alpha\rangle=1$. Therefore, $\omega=\alpha / 2$ and $\langle\omega, \omega\rangle=1 / 2$. We identify points $x$ of $E_{1} \equiv \mathbb{R}$ with $\theta \omega$. The Weyl group $W\left(A_{1}\right)$ 
consists of two elements 1 and $r_{\alpha}$ and $\operatorname{det} r_{\alpha}=-1$. The even Weyl group $W_{e}\left(A_{1}\right)$ consists of

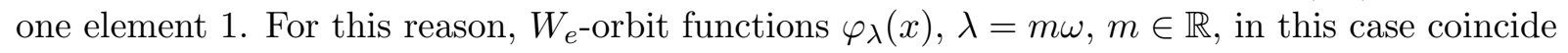
with exponential functions:

$$
E_{\lambda}(x)=e^{2 \pi \mathrm{i}\langle m \omega, \theta \omega\rangle}=e^{\pi \mathrm{i} m \theta} .
$$

Note that for the symmetric and antisymmetric orbit functions $\phi_{\lambda}(x)$ and $\varphi_{\lambda}(x)$ we have

$$
\phi_{\lambda}(x)=2 \cos (\pi m \theta), \quad \varphi_{\lambda}(x)=2 \mathrm{i} \sin (\pi m \theta) .
$$

Therefore, $\phi_{\lambda}(x)=E_{\lambda}(x)+E_{-\lambda}(x)$ and $\varphi_{\lambda}(x)=E_{\lambda}(x)-E_{-\lambda}(x)$.

\section{$4.2 \quad E$-orbit functions of $A_{2}$}

Put $\lambda=a \omega_{1}+b \omega_{2} \equiv(a b)$ with $a>0, b>0$. Then for $\varphi_{\lambda}(x) \equiv \varphi_{(a b)}(x)$ we receive from (4.3) that

$$
\begin{aligned}
& E_{(a b)}(x)=e^{2 \pi i\langle(a b), x\rangle}+e^{2 \pi i\langle(b-a-b), x\rangle}+e^{2 \pi i\langle(-a-b a), x\rangle}, \\
& \left.E_{(-a} a+b\right)(x)=e^{2 \pi i\langle(-a a+b), x\rangle}+e^{2 \pi i\langle(a+b-b), x\rangle}+e^{2 \pi i\langle(-b-a), x\rangle} .
\end{aligned}
$$

Using the representation $x=\psi_{1} \alpha_{1}+\psi_{2} \alpha_{2}$, one obtains

$$
\begin{aligned}
& E_{(a b)}(x)=e^{2 \pi i\left(a \psi_{1}+b \psi_{2}\right)}+e^{2 \pi i\left(b \psi_{1}-(a+b) \psi_{2}\right)}+e^{2 \pi i\left((-a-b) \psi_{1}+a \psi_{2}\right)}, \\
& E_{(-a \quad a+b)}(x)=e^{2 \pi i\left(-a \psi_{1}+(a+b) \psi_{2}\right)}+e^{2 \pi i\left((a+b) \psi_{1}-b \psi_{2}\right)}+e^{2 \pi i\left(-b \psi_{1}-a \psi_{2}\right)} .
\end{aligned}
$$

An expression for $E_{(a b)}(x)$ depends on a choice of coordinate systems for $\lambda$ and $x$. Setting $x=\theta_{1} \omega_{1}+\theta_{2} \omega_{2}$ and $\lambda$ as before, we get

$$
\begin{aligned}
& E_{(a b)}(x)=e^{\frac{2 \pi i}{3}\left((2 a+b) \theta_{1}+(a+2 b) \theta_{2}\right)}+e^{-\frac{2 \pi i}{3}\left((a-b) \theta_{1}+(2 a+b) \theta_{2}\right)}+e^{-\frac{2 \pi i}{3}\left((a+2 b) \theta_{1}+(-a+b) \theta_{2}\right)}, \\
& \left.E_{(-a} a+b\right) \\
& (x)=e^{\frac{2 \pi i}{3}\left((-a+b) \theta_{1}+(a+2 b) \theta_{2}\right)}+e^{\frac{2 \pi i}{3}\left((2 a+b) \theta_{1}+(a-b) \theta_{2}\right)}+e^{-\frac{2 \pi i}{3}\left((a+2 b) \theta_{1}+(2 a+b) \theta_{2}\right)} .
\end{aligned}
$$

Similarly one finds that $E_{(a 0)}(x)$ and $E_{(0 b)}(x)$ are of the form

$$
\begin{aligned}
& E_{(a 0)}(x)=e^{\frac{2 \pi i}{3} a\left(2 \theta_{1}+\theta_{2}\right)}+e^{\frac{2 \pi i}{3} a\left(-\theta_{1}+\theta_{2}\right)}+e^{\frac{2 \pi i}{3} a\left(-\theta_{1}-2 \theta_{2}\right)}, \\
& E_{(0 b)}(x)=e^{\frac{2 \pi i}{3} b\left(\theta_{1}+2 \theta_{2}\right)}+e^{\frac{2 \pi i}{3} b\left(\theta_{1}-\theta_{2}\right)}+e^{\frac{2 \pi i}{3} b\left(-2 \theta_{1}-\theta_{2}\right)}
\end{aligned}
$$

Note that the pairs $E_{(a b)}(x)+E_{(b a)}(x)$ are always real functions.

\section{3 $E$-orbit functions of $C_{2}$ and $G_{2}$}

Putting again $\lambda=a \omega_{1}+b \omega_{2}=(a b), x=\theta_{1} \omega_{1}+\theta_{2} \omega_{2}$ and using the matrices $S$ from (2.6), which are of the form

$$
S\left(C_{2}\right)=\frac{1}{2}\left(\begin{array}{ll}
1 & 1 \\
1 & 2
\end{array}\right), \quad S\left(G_{2}\right)=\frac{1}{6}\left(\begin{array}{ll}
6 & 3 \\
3 & 2
\end{array}\right),
$$

we find the orbit functions $E_{(a b)}(x)$ for $C_{2}$ and $G_{2}$ with $a>0$ and $b>0$ :

$$
\begin{array}{ll}
C_{2}: \quad & E_{(a b)}(x)=2 \cos \pi\left((a+b) \theta_{1}+(a+2 b) \theta_{2}\right)+2 \cos \pi\left(b \theta_{1}-a \theta_{2}\right), \\
& \left.E_{(-a} a+b\right) \\
G_{2}: & E_{(a b)}(x)=2 \cos \pi\left(b \theta_{1}+(a+2 b) \theta_{2}\right)+2 \cos \pi\left((a+b) \theta_{1}+a \theta_{2}\right), \\
& \left.=2 \cos \pi(2 a+b) \theta_{1}+\left(a+\frac{2}{3} b\right) \theta_{2}\right)
\end{array}
$$




$$
\begin{aligned}
+2 & \cos \pi\left((a+b) \theta_{1}+\frac{1}{3} b \theta_{2}\right)+2 \cos \pi\left(a \theta_{1}+\left(a+\frac{1}{3} b\right) \theta_{2}\right), \\
E_{(-a 3 a+b)}(x)= & 2 \cos \pi\left((a+b) \theta_{1}+\left(a+\frac{2}{3} b\right) \theta_{2}\right) \\
& +2 \cos \pi\left((2 a+b) \theta_{1}+\left(a+\frac{1}{3} b\right) \theta_{2}\right)+2 \cos \pi\left(a \theta_{1}-\frac{1}{3} b \theta_{2}\right) .
\end{aligned}
$$

When one of the numbers $a$ and $b$ vanishes, then we have

$$
\begin{aligned}
C_{2}: & E_{(a 0)}(x)=2 \cos \pi a\left(\theta_{1}+\theta_{2}\right)+2 \cos \pi a \theta_{2}, \\
& E_{(0 b)}(x)=2 \cos \pi b\left(\theta_{1}+2 \theta_{2}\right)+2 \cos \pi b \theta_{1}, \\
G_{2}: \quad & E_{(a 0)}(x)=2 \cos \pi a\left(2 \theta_{1}+\theta_{2}\right)+2 \cos \pi a\left(\theta_{1}+\theta_{2}\right)+2 \cos \pi a \theta_{1}, \\
& E_{(0 b)}(x)=2 \cos \pi b\left(\theta_{1}+\frac{2}{3} \theta_{2}\right)+2 \cos \pi b\left(\theta_{1}+\frac{1}{3} \theta_{2}\right)+2 \cos \pi \frac{1}{3} b \theta_{2} .
\end{aligned}
$$

As we see, $E$-functions for $C_{2}$ and $G_{2}$ are real.

\section{$4.4 E$-orbit functions of $A_{n}$}

It is difficult to write down an explicit form of $E$-orbit functions for $A_{n}, B_{n}, C_{n}$ and $D_{n}$ in coordinates with respect to the $\omega$ - or $\alpha$-bases. For this reason, for these cases we use the orthogonal coordinate systems, described in Section 3.

Let $\lambda=\left(m_{1}, m_{2}, \ldots, m_{n+1}\right)$ be a strictly dominant element for $A_{n}$ in orthogonal coordinates described in Subsection 3.3. Then $m_{1}>m_{2}>\cdots>m_{n+1}$. The Weyl group in this case coincides with the symmetric group $S_{n+1}$ and the even Weyl group coincides with the alternating subgroup $S_{n+1}^{e}$ of $S_{n+1}$. The $W_{e}$-orbit $O_{e}(\lambda)$ consists of points $(w \lambda), w \in W_{e}$. Representing points $x \in E_{n+1}$ in the orthogonal coordinate system, $x=\left(x_{1}, x_{2}, \ldots, x_{n+1}\right)$, and using formula (4.3) we find that

$$
E_{\lambda}(x)=\sum_{w \in S_{n+1}^{e}} e^{2 \pi \mathrm{i}\left\langle w\left(m_{1}, \ldots, m_{n+1}\right),\left(x_{1}, \ldots, x_{n+1}\right)\right\rangle}=\sum_{w \in S_{n+1}^{e}} e^{2 \pi \mathrm{i}\left((w \lambda)_{1} x_{1}+\cdots+(w \lambda)_{n+1} x_{n+1}\right)},
$$

where $(w \lambda)_{1},(w \lambda)_{2}, \ldots,(w \lambda)_{n+1}$ are the orthogonal coordinates of the point $w \lambda$.

The second type of $E$-orbit functions correspond to elements $\lambda=\left(m_{1}, m_{2}, \ldots, m_{n+1}\right)$, for which $m_{2}>m_{1}>\cdots>m_{n+1}$. For this case the $E$-orbit functions are given by the same formula (4.18).

If $\lambda=\left(m_{1}, m_{2}, \ldots, m_{n+1}\right)$ is dominant but not strictly dominant (that is, some of $m_{i}$ are coinciding), then the corresponding $E$-orbit function is equal to the symmetric orbit function $\phi_{\left(m_{1}, m_{2}, \ldots, m_{n+1}\right)}(x)$ and, thus, we have

$$
\begin{aligned}
E_{\lambda}(x) & =\sum_{w \in S_{n+1} / S_{\lambda}} e^{2 \pi \mathrm{i}\left\langle w\left(m_{1}, \ldots, m_{n+1}\right),\left(x_{1}, \ldots, x_{n+1}\right)\right\rangle} \\
& =\sum_{w \in S_{n+1} / S_{\lambda}} e^{2 \pi \mathrm{i}\left((w \lambda)_{1} x_{1}+\cdots+(w \lambda)_{n+1} x_{n+1}\right)},
\end{aligned}
$$

where $S_{\lambda}$ is a subgroup of element of $S_{n+1}$ leaving $\lambda$ invariant.

Note that the element $-\left(m_{n+1}, m_{n}, \ldots, m_{1}\right)$ is strictly dominant if the element $\left(m_{1}, m_{2}, \ldots\right.$, $\left.m_{n+1}\right)$ is strictly dominant. In the Weyl group $W\left(A_{n}\right)$ there exists an element $w_{0}$ such that

$$
w_{0}\left(m_{1}, m_{2}, \ldots, m_{n+1}\right)=\left(m_{n+1}, m_{n}, \ldots, m_{1}\right) .
$$

Moreover, we have

$$
\begin{array}{lrrrrr}
\operatorname{det} w_{0} & =1 & \text { for } & A_{4 k-1} & \text { and } & A_{4 k}, \\
\operatorname{det} w_{0} & =-1 & \text { for } & A_{4 k+1} & \text { and } & A_{4 k+2} .
\end{array}
$$

This means that $w_{0} \in W_{e}$ for $A_{4 k-1}$ and $A_{4 k}$ and $w_{0} \notin W_{e}$ for $A_{4 k+1}$ and $A_{4 k+2}$. 
It follows from here that for $A_{4 k-1}$ and $A_{4 k}$ in the expressions for the orbit functions $E_{\left(m_{1}, m_{2}, \ldots, m_{n+1}\right)}(x)$ and $E_{-\left(m_{n+1}, m_{n}, \ldots, m_{1}\right)}(x)$ there are summands

$$
e^{2 \pi \mathrm{i}\left\langle w_{0} \lambda, x\right\rangle}=e^{2 \pi \mathrm{i}\left(m_{n+1} x_{1}+\cdots+m_{1} x_{n+1}\right)} \quad \text { and } \quad e^{-2 \pi \mathrm{i}\left(m_{n+1} x_{1}+\cdots+m_{1} x_{n+1}\right)},
$$

respectively, which are complex conjugate to each other.

Similarly, for $A_{4 k-1}$ and $A_{4 k}$, in the expressions (4.18) for the functions $E_{\left(m_{1}, m_{2}, \ldots, m_{n+1}\right)}(x)$ and $E_{-\left(m_{n+1}, m_{n}, \ldots, m_{1}\right)}(x)$ all other summands are pairwise complex conjugate. Therefore,

$$
E_{\left(m_{1}, m_{2}, \ldots, m_{n+1}\right)}(x)=\overline{E_{-\left(m_{n+1}, m_{n}, \ldots, m_{1}\right)}(x)}
$$

for $n=4 k-1,4 k$. If to use for $\lambda$ the coordinates $\lambda_{i}=\left\langle\lambda, \alpha_{i}^{\vee}\right\rangle$ in the $\omega$-basis instead of the orthogonal coordinates $m_{j}$, then this equation can be written as

$$
E_{\left(\lambda_{1}, \ldots, \lambda_{n}\right)}(x)=\overline{E_{\left(\lambda_{n}, \ldots, \lambda_{1}\right)}(x)} .
$$

If $n=4 k+1$ or $n=4 k+2$, then the $E$-orbit function $E_{-\left(m_{n+1}, m_{n}, \ldots, m_{1}\right)}(x)$ belongs to the second type of $E$-orbit functions. In this case the orbit functions $E_{\left(m_{1}, m_{2}, \ldots, m_{n+1}\right)}(x)$ and $E_{-\left(m_{n+1}, m_{n}, \ldots, m_{1}\right)}(x)$ have no common summands. In this case we have

$$
E_{-r_{\alpha}\left(m_{n+1}, m_{n}, \ldots, m_{1}\right)}(x)=\overline{E_{\left(m_{1}, m_{2}, \ldots, m_{n+1}\right)}(x)},
$$

where $\alpha$ is a positive root of our root system.

According to (4.21), if

$$
\left(m_{1}, m_{2}, \ldots, m_{n+1}\right)=-\left(m_{n+1}, m_{n}, \ldots, m_{1}\right)
$$

(that is, the element $\lambda$ has in the $\omega$-basis the coordinates $\left.\left(\lambda_{1}, \lambda_{2}, \ldots, \lambda_{2}, \lambda_{1}\right)\right)$, then the E-orbit function $E_{\lambda}$ is real for $n=4 k-1,4 k$. This orbit function can be represented as a sum of cosines of angles.

It is know from Proposition 2 in [7] that in the orthogonal coordinates antisymmetric orbit functions $\varphi_{\left(m_{1}, m_{2}, \ldots, m_{n+1}\right)}(x), m_{1}>m_{2}>\cdots>m_{n+1}$, of $A_{n}$ can be represented as determinants of certain matrices:

$$
\begin{aligned}
\varphi_{\left(m_{1}, m_{2}, \ldots, m_{n+1}\right)}(x) & =\operatorname{det}\left(e^{2 \pi \mathrm{i} m_{i} x_{j}}\right)_{i, j=1}^{n+1} \\
& \equiv \operatorname{det}\left(\begin{array}{cccc}
e^{2 \pi \mathrm{i} m_{1} x_{1}} & e^{2 \pi \mathrm{i} m_{1} x_{2}} & \cdots & e^{2 \pi \mathrm{i} m_{1} x_{n+1}} \\
e^{2 \pi \mathrm{i} m_{2} x_{1}} & e^{2 \pi \mathrm{i} m_{2} x_{2}} & \cdots & e^{2 \pi \mathrm{i} m_{2} x_{n+1}} \\
\cdots & \cdots & \cdots & \cdots \\
e^{2 \pi \mathrm{i} m_{n+1} x_{1}} & e^{2 \pi \mathrm{i} m_{n+1} x_{2}} & \cdots & e^{2 \pi \mathrm{i} m_{n+1} x_{n+1}}
\end{array}\right) .
\end{aligned}
$$

It follows from this formula that the corresponding $E$-orbit functions $E_{\left(m_{1}, m_{2}, \ldots, m_{n+1}\right)}(x)$ and $E_{\left(m_{2}, m_{1}, m_{3}, \ldots, m_{n+1}\right)}(x)$ can be represented as

$$
\begin{aligned}
& E_{\left(m_{1}, m_{2}, m_{3}, \ldots, m_{n+1}\right)}(x)=\left[\operatorname{det}\left(e^{2 \pi \mathrm{i} m_{i} x_{j}}\right)_{i, j=1}^{n+1}\right]^{+}, \\
& E_{\left(m_{2}, m_{1}, m_{3}, \ldots, m_{n+1}\right)}(x)=\left[\operatorname{det}\left(e^{2 \pi \mathrm{i} m_{i} x_{j}}\right)_{i, j=1}^{n+1}\right]^{-},
\end{aligned}
$$

where $[\operatorname{det} C]^{+}$and $[\operatorname{det} C]^{-}$mean a parts of the expression for the determinant of $C$ containing all terms with sigh plus and with sign minus, respectively. 


\subsection{E-orbit functions of $B_{n}$}

Let $\lambda=\left(m_{1}, m_{2}, \ldots, m_{n}\right)$ be a strictly dominant element for $B_{n}$ in orthogonal coordinates described in Subsection 3.4. Then $m_{1}>m_{2}>\cdots>m_{n}>0$. The Weyl group $W\left(B_{n}\right)$ consists of permutations of the coordinates $m_{i}$ with sign alternations of some of them. The even Weyl group $W_{e}\left(B_{n}\right)$ consists of those elements of $w \in W\left(B_{n}\right)$ for which $\operatorname{det} w=1$. Representing points $x \in E_{n}$ also in the orthogonal coordinate system, $x=\left(x_{1}, x_{2}, \ldots, x_{n}\right)$, and using formula (4.3) we find that the antisymmetric orbit function of $W\left(B_{n}\right)$, corresponding to element $\lambda$, coincides with

$$
\begin{aligned}
\varphi_{\lambda}(x) & =\sum_{\varepsilon_{i}= \pm 1} \sum_{w \in S_{n}}(\operatorname{det} w) \varepsilon_{1} \varepsilon_{2} \cdots \varepsilon_{n} e^{2 \pi \mathrm{i}\left\langle w\left(\varepsilon_{1} m_{1}, \ldots, \varepsilon_{n} m_{n}\right),\left(x_{1}, \ldots, x_{n}\right)\right\rangle} \\
& =\sum_{\varepsilon_{i}= \pm 1} \sum_{w \in S_{n}}(\operatorname{det} w) \varepsilon_{1} \varepsilon_{2} \cdots \varepsilon_{n} e^{2 \pi \mathrm{i}\left((w(\varepsilon \lambda))_{1} x_{1}+\cdots+(w(\varepsilon \lambda))_{n} x_{n}\right)},
\end{aligned}
$$

where $(w(\varepsilon \lambda))_{1}, \ldots,(w(\varepsilon \lambda))_{n}$ are the orthogonal coordinates of the points $w(\varepsilon \lambda)$ if $\varepsilon \lambda=\left(\varepsilon_{1} m_{1}\right.$, $\left.\ldots, \varepsilon_{n} m_{n}\right)$.

In order to obtain the corresponding $E$-orbit function $E_{\lambda}(x)$ we have to take in the expression (4.26) only those terms, for which $(\operatorname{det} w) \varepsilon_{1} \varepsilon_{2} \cdots \varepsilon_{n}=1$. It is easy to see that

$$
\begin{aligned}
E_{\lambda}(x)= & \sum_{\varepsilon_{i}= \pm 1}^{\prime} \sum_{w \in S_{n}^{e}} e^{2 \pi \mathrm{i}\left((w(\varepsilon \lambda))_{1} x_{1}+\cdots+(w(\varepsilon \lambda))_{n} x_{n}\right)} \\
& +\sum_{\varepsilon_{i}= \pm 1}^{\prime \prime} \sum_{w \in S_{n} \backslash S_{n}^{e}} e^{2 \pi \mathrm{i}\left((w(\varepsilon \lambda))_{1} x_{1}+\cdots+(w(\varepsilon \lambda))_{n} x_{n}\right)},
\end{aligned}
$$

where $\sum_{\varepsilon_{i}= \pm 1}{ }^{\prime}$ means the sum over $\varepsilon_{i}$ such that $\varepsilon_{1} \varepsilon_{2} \cdots \varepsilon_{n}=1$ and $\sum_{\varepsilon_{i}= \pm 1}$ "' means the sum over $\varepsilon_{i}$ such that $\varepsilon_{1} \varepsilon_{2} \cdots \varepsilon_{n}=-1$. The notation $S_{n} \backslash S_{n}^{e}$ means a complement of $S_{n}$ to $S_{n}^{e}$, where, as before, $S_{n}^{e}$ is the alternating subgroup of $S_{n}$.

For the $E$-orbit function $E_{r_{\alpha} \lambda}(x)$ we respectively have

$$
\begin{aligned}
E_{r_{\alpha} \lambda}(x)= & \sum_{\varepsilon_{i}= \pm 1}^{\prime \prime} \sum_{w \in S_{n}^{e}} e^{2 \pi \mathrm{i}\left((w(\varepsilon \lambda))_{1} x_{1}+\cdots+(w(\varepsilon \lambda))_{n} x_{n}\right)} \\
& +\sum_{\varepsilon_{i}= \pm 1}^{\prime} \sum_{w \in S_{n} \backslash S_{n}^{e}} e^{2 \pi \mathrm{i}\left((w(\varepsilon \lambda))_{1} x_{1}+\cdots+(w(\varepsilon \lambda))_{n} x_{n}\right)},
\end{aligned}
$$

where $\sum_{\varepsilon_{i}= \pm 1}^{\prime}$ and $\sum_{\varepsilon_{i}= \pm 1}^{\prime \prime}$ are such as in (4.27).

In $W\left(B_{n}\right)$ there exists an element $w_{-}$which change signs of all coordinates $m_{i}$. Then $\operatorname{det} w_{-}=1$ if $n=2 k$ and $\operatorname{det} w_{-}=-1$ if $n=2 k+1$. Therefore, for each summand $e^{2 \pi \mathrm{i}\left((w(\varepsilon \lambda))_{1} x_{1}+\cdots+(w(\varepsilon \lambda))_{n} x_{n}\right)}$ in the expressions (4.27) for the $E$-orbit function $E_{\left(m_{1}, m_{2}, \ldots, m_{n}\right)}(x)$ there exists exactly one summand complex conjugate to it, $e^{-2 \pi \mathrm{i}\left(\left((w(\varepsilon \lambda))_{1} x_{1}+\cdots+(w(\varepsilon \lambda))_{n} x_{n}\right)\right.}$, if $n=2 k$. This means that E-orbit functions of $B_{n}$ are real if $n=2 k$. These orbit functions can be represented as sums of cosines of the corresponding angles. If $n=2 k+1$, then the expression $e^{-2 \pi \mathrm{i}\left(\left((w(\varepsilon \lambda))_{1} x_{1}+\cdots+(w(\varepsilon \lambda))_{n} x_{n}\right)\right.}$ is not contained in the $E$-orbit function $E_{\left(m_{1}, m_{2}, \ldots, m_{n}\right)}(x)$. Therefore, in this case this expression belongs to the $E$-orbit function of the second type, that is, to the $E$-orbit function $E_{\left(m_{2}, m_{1}, m_{3}, \ldots, m_{n}\right)}(x)$. We conclude that when $n=2 k+1$, then the $E$-orbit functions $E_{\lambda}(x)$ and $E_{r_{\alpha} \lambda}(x)$ are pairwise complex conjugate to each other.

If $\lambda$ is dominant but not strictly dominant, then the $E$-orbit function $E_{\lambda}(x)$ coincides with the symmetric orbit function $\phi_{\lambda}(x)$ and we have

$$
E_{\lambda}(x)=\sum_{\varepsilon_{i}= \pm 1} \sum_{w \in S_{n} / S_{\lambda}} e^{2 \pi \mathrm{i}\left\langle w\left(\varepsilon_{1} m_{1}, \ldots, \varepsilon_{n} m_{n}\right),\left(x_{1}, \ldots, x_{n}\right)\right\rangle}
$$




$$
=\sum_{\varepsilon_{i}= \pm 1} \sum_{w \in S_{n} / S_{\lambda}} e^{2 \pi \mathrm{i}\left((w(\varepsilon \lambda))_{1} x_{1}+\cdots+(w(\varepsilon \lambda))_{n} x_{n}\right)} .
$$

The summation here is over those $\varepsilon_{i}= \pm 1$ for which $m_{i} \neq 0$.

\subsection{E-orbit functions of $C_{n}$}

Let $\lambda=\left(m_{1}, m_{2}, \ldots, m_{n}\right)$ be a strictly dominant element for $C_{n}$ in the orthogonal coordinates described in Subsection 3.5. Then $m_{1}>m_{2}>\cdots>m_{n}>0$. Representing points $x \in E_{n}$ also in the orthogonal coordinate system, $x=\left(x_{1}, x_{2}, \ldots, x_{n}\right)$, we find that the antisymmetric orbit function of $W\left(C_{n}\right)$, corresponding to $\lambda$, coincides with

$$
\begin{aligned}
\varphi_{\lambda}(x) & =\sum_{\varepsilon_{i}= \pm 1} \sum_{w \in S_{n}}(\operatorname{det} w) \varepsilon_{1} \varepsilon_{2} \ldots \varepsilon_{n} e^{2 \pi \mathrm{i}\left\langle w\left(\varepsilon_{1} m_{1}, \ldots, \varepsilon_{n} m_{n}\right),\left(x_{1}, \ldots, x_{n}\right)\right\rangle} \\
& =\sum_{\varepsilon_{i}= \pm 1} \sum_{w \in S_{n}}(\operatorname{det} w) \varepsilon_{1} \varepsilon_{2} \cdots \varepsilon_{n} e^{2 \pi \mathrm{i}\left((w(\varepsilon \lambda))_{1} x_{1}+\cdots+(w(\varepsilon \lambda))_{n} x_{n}\right)},
\end{aligned}
$$

where, as above, $(w(\varepsilon \lambda))_{1}, \ldots,(w(\varepsilon \lambda))_{n}$ are the orthogonal coordinates of the points $w(\varepsilon \lambda)$, where $\varepsilon \lambda=\left(\varepsilon_{1} m_{1}, \ldots, \varepsilon_{n} m_{n}\right)$.

In order to obtain the corresponding $E$-orbit function $E_{\lambda}(x)$ we have to take in the expression (4.30) only those terms, for which $(\operatorname{det} w) \varepsilon_{1} \varepsilon_{2} \cdots \varepsilon_{n}=1$. It is easy to see that

$$
\begin{aligned}
E_{\lambda}(x)= & \sum_{\varepsilon_{i}= \pm 1}^{\prime} \sum_{w \in S_{n}^{e}} e^{2 \pi \mathrm{i}\left((w(\varepsilon \lambda))_{1} x_{1}+\cdots+(w(\varepsilon \lambda))_{n} x_{n}\right)} \\
& +\sum_{\varepsilon_{i}= \pm 1}^{\prime \prime} \sum_{w \in S_{n} \backslash S_{n}^{e}} e^{2 \pi \mathrm{i}\left((w(\varepsilon \lambda))_{1} x_{1}+\cdots+(w(\varepsilon \lambda))_{n} x_{n}\right)},
\end{aligned}
$$

where $\sum_{\varepsilon_{i}= \pm 1}{ }^{\prime}$ means the sum over $\varepsilon_{i}$ such that $\varepsilon_{1} \varepsilon_{2} \cdots \varepsilon_{n}=1$ and $\sum_{\varepsilon_{i}= \pm 1}$ "' means the sum over $\varepsilon_{i}$ such that $\varepsilon_{1} \varepsilon_{2} \cdots \varepsilon_{n}=-1$. The notation $S_{n} \backslash S_{n}^{e}$ means a complement of $S_{n}$ to $S_{n}^{e}$. For the $E$-orbit functions $E_{r_{\alpha} \lambda}(x)$ we have the expression

$$
\begin{aligned}
E_{\lambda}(x)= & \sum_{\varepsilon_{i}= \pm 1}^{\prime \prime} \sum_{w \in S_{n}^{e}} e^{2 \pi \mathrm{i}\left((w(\varepsilon \lambda))_{1} x_{1}+\cdots+(w(\varepsilon \lambda))_{n} x_{n}\right)} \\
& +\sum_{\varepsilon_{i}= \pm 1}^{\prime} \sum_{w \in S_{n} \backslash S_{n}^{e}} e^{2 \pi \mathrm{i}\left((w(\varepsilon \lambda))_{1} x_{1}+\cdots+(w(\varepsilon \lambda))_{n} x_{n}\right)}
\end{aligned}
$$

where $\sum_{\varepsilon_{i}= \pm 1}^{\prime}$ and $\sum_{\varepsilon_{i}= \pm 1}^{\prime \prime}$ are such as in (4.31).

In $W\left(C_{n}\right)$ there exists an element $w_{-}$which change signs of all coordinates $m_{i}$. Then $\operatorname{det} w_{-}=1$ if $n=2 k$ and $\operatorname{det} w_{-}=-1$ if $n=2 k+1$. Therefore, for each summand $e^{2 \pi \mathrm{i}\left((w(\varepsilon \lambda))_{1} x_{1}+\cdots+(w(\varepsilon \lambda))_{n} x_{n}\right)}$ in the expressions (4.31) for the $E$-orbit function $E_{\left(m_{1}, m_{2}, \ldots, m_{n}\right)}(x)$ there exists exactly one summand complex conjugate to it, $e^{-2 \pi \mathrm{i}\left(\left((w(\varepsilon \lambda))_{1} x_{1}+\cdots+(w(\varepsilon \lambda))_{n} x_{n}\right)\right.}$, if $n=2 k$. This means that E-orbit functions of $C_{n}$ are real if $n=2 k$. This orbit function of $C_{n}$ can be represented as a sum of cosines of the corresponding angles. If $n=2 k+1$, then the expression $e^{-2 \pi \mathrm{i}\left(\left((w(\varepsilon \lambda))_{1} x_{1}+\cdots+(w(\varepsilon \lambda))_{n} x_{n}\right)\right.}$ is contained in the $E$-orbit function $E_{r_{\alpha}\left(m_{1}, m_{2}, \ldots, m_{n}\right)}(x)$. We conclude that when $n=2 k+1$, then the $E$-orbit functions $E_{\lambda}(x)$ and $E_{r_{\alpha} \lambda}(x)$ are pairwise complex conjugate to each other.

If $\lambda$ is dominant but not strictly dominant, then the $E$-orbit function $E_{\lambda}(x)$ coincides with the symmetric orbit function $\phi_{\lambda}(x)$ and we have

$$
E_{\lambda}(x)=\sum_{\varepsilon_{i}= \pm 1} \sum_{w \in S_{n} / S_{\lambda}} e^{2 \pi \mathrm{i}\left\langle w\left(\varepsilon_{1} m_{1}, \ldots, \varepsilon_{n} m_{n}\right),\left(x_{1}, \ldots, x_{n}\right)\right\rangle}
$$




$$
=\sum_{\varepsilon_{i}= \pm 1} \sum_{w \in S_{n} / S_{\lambda}} e^{2 \pi \mathrm{i}\left((w(\varepsilon \lambda))_{1} x_{1}+\cdots+(w(\varepsilon \lambda))_{n} x_{n}\right)}
$$

where the summation is over those $\varepsilon_{i}= \pm 1$ for which $m_{i} \neq 0$.

Note that in the orthogonal coordinates expressions for the $E$-orbit functions $E_{\left(m_{1}, m_{2}, \ldots, m_{n}\right)}(x)$ of $C_{n}$ coincide with the expressions for the corresponding $E$-orbit functions $E_{\left(m_{1}, m_{2}, \ldots, m_{n}\right)}(x)$ of $B_{n}$. However, $\alpha$-coordinates of the element $\left(m_{1}, m_{2}, \ldots, m_{n}\right)$ for $C_{n}$ do not coincide with $\alpha$-coordinates of the element $\left(m_{1}, m_{2}, \ldots, m_{n}\right)$ for $B_{n}$, that is, in $\alpha$-coordinates expressions for the corresponding $E$-orbit functions of $B_{n}$ and $C_{n}$ are different.

\subsection{E-orbit functions of $D_{n}$}

Let $\lambda=\left(m_{1}, m_{2}, \ldots, m_{n}\right)$ be a strictly dominant element for $D_{n}$ in the orthogonal coordinates described in Subsection 3.6. Then $m_{1}>m_{2}>\cdots>m_{n-1}>\left|m_{n}\right|$. The Weyl group $W\left(D_{n}\right)$ consists of permutations of the coordinates with sign alternations of even number of them. The even Weyl group $W_{e}\left(D_{n}\right)$ consists of even permutations of the coordinates with sign alternations of even number of them.

Representing points $x \in E_{n}$ also in the orthogonal coordinate system, $x=\left(x_{1}, x_{2}, \ldots, x_{n}\right)$, and using formula (4.3) we find that the antisymmetric orbit function $\varphi_{\lambda}(x)$ of $D_{n}$ is given by the formula

$$
\begin{aligned}
\varphi_{\lambda}(x) & =\sum_{\varepsilon_{i}= \pm 1}^{\prime} \sum_{w \in S_{n}}(\operatorname{det} w) e^{2 \pi \mathrm{i}\left\langle w\left(\varepsilon_{1} m_{1}, \ldots, \varepsilon_{n} m_{n}\right),\left(x_{1}, \ldots, x_{n}\right)\right\rangle} \\
& =\sum_{\varepsilon_{i}= \pm 1}^{\prime} \sum_{w \in S_{n}}(\operatorname{det} w) e^{2 \pi \mathrm{i}\left((w(\varepsilon \lambda))_{1} x_{1}+\cdots+(w(\varepsilon \lambda))_{n} x_{n}\right)}
\end{aligned}
$$

where $(w(\varepsilon \lambda))_{1}, \ldots,(w(\varepsilon \lambda))_{n}$ are the orthogonal coordinates of the points $w(\varepsilon \lambda)$ and the prime at the sum symbol means that the summation is over values of $\varepsilon_{i}$ with even number of sign minus. We have taken into account that an alternation of coordinates without any permutation does not change a determinant.

Therefore, if $\lambda$ is strictly dominant, then the $E$-orbit function $E_{\lambda}(x)$ is of the form

$$
E_{\lambda}(x)=\sum_{\varepsilon_{i}= \pm 1}^{\prime} \sum_{w \in S_{n}^{e}} e^{2 \pi \mathrm{i}\left((w(\varepsilon \lambda))_{1} x_{1}+\cdots+(w(\varepsilon \lambda))_{n} x_{n}\right)}
$$

where $S_{n}^{e}$ is the even part of the symmetric group $S_{n}$ and the first sum is such as in (4.34). For the corresponding $E$-orbit functions $E_{r_{\alpha} \lambda}(x)$ we have

$$
E_{r_{\alpha} \lambda}(x)=\sum_{\varepsilon_{i}= \pm 1}^{\prime} \sum_{w \in S_{n} \backslash S_{n}^{e}} e^{2 \pi \mathrm{i}\left((w(\varepsilon \lambda))_{1} x_{1}+\cdots+(w(\varepsilon \lambda))_{n} x_{n}\right)}
$$

where the first sum is such as in (4.35).

Let $m_{n} \neq 0$. Then in the expressions (4.35) for the $E$-orbit function $E_{\left(m_{1}, m_{2}, \ldots, m_{n}\right)}(x)$ of $D_{n=2 k}$ for each summand $e^{2 \pi \mathrm{i}\left((w(\varepsilon \lambda))_{1} x_{1}+\cdots+(w(\varepsilon \lambda))_{n} x_{n}\right)}$ there exists exactly one summand complex conjugate to it. This means that these E-orbit functions of $D_{2 k}$ are real. Each orbit function of $D_{2 k}$ can be represented as a sum of cosines of the corresponding angles.

It is also proved by using (4.35) that for $m_{n} \neq 0$ the E-orbit functions $E_{\left(m_{1}, \ldots, m_{2 k}, m_{2 k+1}\right)}(x)$ and $E_{\left(m_{1}, \ldots, m_{2 k},-m_{2 k+1}\right)}(x)$ of $D_{2 k+1}$ are complex conjugate.

If $m_{n}=0$, then it follows from (4.35) that $E$-orbit functions $E_{\lambda}(x)$ of $D_{n}$ are real and can be represented as a sum of cosines of certain angles. 
For dominant, but not strictly dominant, elements $\lambda$ the $E$-orbit functions coincide with the corresponding symmetric orbit functions and we have

$$
\begin{aligned}
E_{\lambda}(x) & =\sum_{\varepsilon_{i}= \pm 1}^{\prime} \sum_{w \in S_{n} / S_{\lambda}} e^{2 \pi \mathrm{i}\left\langle w\left(\varepsilon_{1} m_{1}, \ldots, \varepsilon_{n} m_{n}\right),\left(x_{1}, \ldots, x_{n}\right)\right\rangle} \\
& =\sum_{\varepsilon_{i}= \pm 1}^{\prime} \sum_{w \in S_{n} / S_{\lambda}} e^{2 \pi \mathrm{i}\left((w(\varepsilon \lambda))_{1} x_{1}+\cdots+(w(\varepsilon \lambda))_{n} x_{n}\right)}
\end{aligned}
$$

where $S_{\lambda}$ is the subgroup of $S_{n}$ consisting of elements leaving $\lambda$ invariant.

Note that in the expressions (4.37) for the orbit function $E_{\left(m_{1}, m_{2}, \ldots, m_{n}\right)}(x)$ of $D_{2 k}$ for each summand $e^{2 \pi \mathrm{i}\left((w(\varepsilon \lambda))_{1} x_{1}+\cdots+(w(\varepsilon \lambda))_{n} x_{n}\right)}$ there exists exactly one summand complex conjugate to it. This means that all orbit functions of $D_{2 k}$ are real. Each orbit function of $D_{2 k}$ can be represented as a sum of cosines of the corresponding angles.

It also follows from (4.37) that for $D_{2 k+1}$ the E-orbit function $E_{\left(m_{1}, m_{2}, \ldots, m_{n}\right)}(x)$ is real if and only if the condition $m_{2 k+1}=0$ is fulfilled. The E-orbit functions $E_{\left(m_{1}, \ldots, m_{2 k}, m_{2 k+1}\right)}(x)$ and $E_{\left(m_{1}, \ldots, m_{2 k},-m_{2 k+1}\right)}(x)$ of $D_{2 k+1}$ are complex conjugate.

\section{$5 \quad$ Properties of $\boldsymbol{E}$-orbit functions}

\subsection{Invariance with respect to the even Weyl group}

Since the scalar product $\langle\cdot, \cdot\rangle$ in $E_{n}$ is invariant with respect to the Weyl group $W$, that is,

$$
\langle w x, w y\rangle=\langle x, y\rangle, \quad w \in W, \quad x, y \in E_{n},
$$

it is invariant with respect to the even Weyl group $W_{e}$, which is a subgroup of $W$. It follows from here that $E$-orbit functions $E_{\lambda}$ for strictly dominant elements $\lambda$ are invariant with respect to $W_{e}$, that is,

$$
E_{\lambda}\left(w^{\prime} x\right)=E_{\lambda}(x), \quad w^{\prime} \in W_{e} .
$$

Indeed, this relation is equivalent to the relation $\hat{E}_{\lambda}\left(w^{\prime} x\right)=\hat{E}_{\lambda}(x)$ for the functions (4.5). For $\hat{E}_{\lambda}(x)$ we have

$$
\hat{E}_{\lambda}\left(w^{\prime} x\right)=\sum_{w \in W_{e}} e^{2 \pi \mathrm{i}\left\langle w \lambda, w^{\prime} x\right\rangle}=\sum_{w \in W_{e}} e^{2 \pi \mathrm{i}\left\langle w^{\prime-1} w \lambda, x\right\rangle}=\sum_{w \in W_{e}}(\operatorname{det} w) e^{2 \pi \mathrm{i}\langle w \lambda, x\rangle}=\hat{E}_{\lambda}(x)
$$

since $w^{\prime-1} w$ runs over the whole group $W_{e}$ when $w$ runs over $W_{e}$.

In the same way it is shown that the corresponding $E$-orbit functions $E_{r_{\alpha} \lambda}(x)$, where $\lambda$ is as above and $\alpha$ is a root, is invariant with respect to $W_{e}$.

If $\lambda$ is dominant, but not strictly dominant, then $E_{\lambda}(x)$ coincides with the corresponding symmetric orbit function, which is invariant with respect to the Weyl group $W$, and therefore with respect to $W_{e}$.

\subsection{Invariance with respect to the even affine Weyl group}

If $\lambda$ belongs to the set of dominant integral elements $P_{+}$or to $r_{\alpha} P_{+}$, then $E_{\lambda}(x)$ is invariant with respect to the even affine group $W_{e}^{\text {aff }}$. Recall that $W_{e}^{\text {aff }}$ is a semidirect product of the even Weyl group $W_{e}$ and the group $\hat{Q}$ of translations by elements of the dual root system $Q^{\vee}$. In order to prove this invariance of $E$-orbit functions $E_{\lambda}(x)$ we need only to prove their invariance with respect to the subgroup $\hat{Q}$. For this we note that for $\mu \in P$ and for $\nu \in Q^{\vee}$ we have

$$
\langle\mu, x+\nu\rangle=\langle\mu, x\rangle+\langle\mu, \nu\rangle=\langle\mu, x\rangle+\text { integer. }
$$


Hence,

$$
E_{\lambda}(x+\nu)=\left|W_{\lambda}\right|^{-1} \sum_{w \in W_{e}} e^{2 \pi \mathrm{i}\langle w \lambda, x+\nu\rangle}=\left|W_{\lambda}\right|^{-1} \sum_{w \in W_{e}} e^{2 \pi \mathrm{i}\langle\lambda, x\rangle}=E_{\lambda}(x),
$$

where $W_{\lambda}$ is the subgroup of $W_{e}$ consisting of elements $w$ such that $w \lambda=\lambda$. Thus, $E_{\lambda}(x)$ is invariant with respect to the even affine Weyl group $W_{e}^{\text {aff }}$.

If $\lambda \notin P$, then $E_{\lambda}$ is not invariant with respect to $W_{e}^{\text {aff }}$. It is invariant only under action by elements of the even Weyl group $W_{e}$.

Due to the invariance of $E$-orbit functions $E_{\lambda}, \lambda \in P_{+}$, with respect to the group $W_{e}^{\text {aff }}$, it is enough to consider them only on the even fundamental domain $F_{e}$ of $W_{e}^{\text {aff }}$ (see Subsection 2.6). Values of $E_{\lambda}$ on other points of $E_{n}$ are determined by using the action of $W_{e}^{\text {aff }}$ on $F_{e}$ or taking a limit.

\subsection{Relation to symmetric and antisymmetric orbit functions}

$E$-orbit functions are closely related to symmetric and antisymmetric orbit functions $\phi_{\lambda}(x)$ and $\varphi_{\lambda}(x)$. We have seen that if $\lambda$ is lying on a wall of the dominant Weyl chamber, then

$$
E_{\lambda}(x)=\phi_{\lambda}(x) .
$$

Since the Weyl group $W$ can be represented as a union of the even Weyl group $W_{e}$ and of the set $r_{\alpha} W_{e}$,

$$
W=W_{e} \bigcup r_{\alpha} W_{e}
$$

then it follows from the definitions of symmetric and antisymmetric orbit functions that for strictly dominant $\lambda$ we have

$$
\begin{aligned}
& \phi_{\lambda}(x)=E_{\lambda}(x)+E_{r_{\alpha} \lambda}(x), \\
& \varphi_{\lambda}(x)=E_{\lambda}(x)-E_{r_{\alpha} \lambda}(x) .
\end{aligned}
$$

It follows from here that for such $\alpha$ one gets

$$
\begin{aligned}
& E_{\lambda}(x)=\frac{1}{2}\left(\phi_{\lambda}(x)+\varphi_{\lambda}(x)\right), \\
& E_{r_{\alpha} \lambda}(x)=\frac{1}{2}\left(\phi_{\lambda}(x)-\varphi_{\lambda}(x)\right) .
\end{aligned}
$$

It is directly derived from (5.3)-(5.6) that the relations

$$
\begin{aligned}
& \phi_{\lambda}^{2}(x)-\varphi_{\lambda}^{2}(x)=4 E_{\lambda}(x) E_{r_{\alpha} \lambda}(x), \\
& \phi_{\lambda}^{2}(x)+\varphi_{\lambda}^{2}(x)=2\left(E_{\lambda}^{2}(x)+E_{r_{\alpha} \lambda}^{2}(x)\right)
\end{aligned}
$$

are true, where $\lambda$ is strictly dominant.

\subsection{Continuity}

An $E$-orbit function $E_{\lambda}(x)$ is a finite sum of exponential functions. Therefore, it is continuous and has continuous derivatives of all orders on the Euclidean space $E_{n}$.

Antisymmetric orbit functions $\varphi_{\lambda}$ vanish on the boundary of the fundamental domain $F(W)$ of the Weyl group $W$. The normal derivative $\partial \mathbf{n}$ of symmetric orbit functions $\phi_{\lambda}$ to the boundary $\partial F(W)$ of the fundamental domain $F(W)$ equals zero. The reason of these properties is (anti)symmetry with respect to the reflections with respect to walls of the dominant Weyl chamber. These reflections do not belong to $W_{e}$ and, therefore, $E$-orbit functions do not have these properties. 


\subsection{Scaling symmetry}

Let $O_{e}(\lambda)$ be an $E$-orbit of $\lambda, \lambda \in D_{+}$. Since $w(c \lambda)=c w(\lambda)$ for any $c \in \mathbb{R}$ and for any $w \in W_{e}$, then the orbit $O_{e}(c \lambda)$ is an orbit consisting of the points $c w \lambda, w \in W_{e}$. Let $E_{\lambda}=\sum_{w \in W_{e}} e^{2 \pi \mathrm{i} w \lambda}$ be the $E$-orbit function for $\lambda \in D_{+}$. Then

$$
E_{c \lambda}(x)=\left|W_{\lambda}\right|^{-1} \sum_{w \in W_{e}} e^{2 \pi \mathrm{i}\langle c w \lambda, x\rangle}=\left|W_{\lambda}\right|^{-1} \sum_{w \in W_{e}} e^{2 \pi \mathrm{i}\langle w \lambda, c x\rangle}=E_{\lambda}(c x) .
$$

The equality $E_{c \lambda}(x)=E_{\lambda}(c x)$ expresses the scaling symmetry of E-orbit functions.

\subsection{Duality}

Due to the invariance of the scalar product $\langle\cdot, \cdot\rangle$ with respect to the even Weyl group $W_{e}$, $\langle w \mu, w y\rangle=\langle\mu, y\rangle$, for $E$-orbit functions $\hat{E}_{\lambda}(x)$ (see Subsection 4.1) we have

$$
\hat{E}_{\lambda}(x)=\sum_{w \in W_{e}} e^{2 \pi \mathrm{i}\left\langle\lambda, w^{-1} x\right\rangle}=\sum_{w \in W_{e}} e^{2 \pi \mathrm{i}\langle\lambda, w x\rangle}=\hat{E}_{x}(\lambda) .
$$

It is easy to see that this relation is also true for $E$-orbit functions $E_{\lambda}(x)$. The relation $E_{\lambda}(x)=$ $E_{x}(\lambda)$ expresses the duality of $E$-orbit functions.

$E$-orbit functions have also the following property

$$
E_{r_{\alpha} \lambda}(x)=E_{\lambda}\left(r_{\alpha} x\right),
$$

where $\alpha$ is a root of the corresponding root system. This relation follows from the fact that $r_{\alpha} W_{e}=W_{e} r_{\alpha}$ and from the equalities

$$
\hat{E}_{r_{\alpha} \lambda}(x)=\sum_{w \in W_{e}} e^{2 \pi \mathrm{i}\left\langle w r_{\alpha} \lambda, x\right\rangle}=\sum_{w \in W_{e}} e^{2 \pi \mathrm{i}\left\langle r_{\alpha} w \lambda, x\right\rangle}=\sum_{w \in W_{e}} e^{2 \pi \mathrm{i}\left\langle w \lambda, r_{\alpha} x\right\rangle}=\hat{E}_{\lambda}\left(r_{\alpha} x\right),
$$

where $\lambda$ is strictly dominant.

\subsection{Orthogonality}

If values of $\lambda$ are integral points lying inside of the fundamental domain $F_{e}$ of the even affine Weyl group $W_{e}^{\text {aff }}$, then the corresponding $E$-orbit functions are orthogonal on the closure $\overline{F_{e}}$ of the fundamental domain $F_{e}$ with respect to the Euclidean measure:

$$
\left|\overline{F_{e}}\right|^{-1} \int_{\overline{F_{e}}} E_{\lambda}(x) \overline{E_{\lambda^{\prime}}(x)} d x=\left|W_{e}\right| \delta_{\lambda \lambda^{\prime}},
$$

where the overbar over $E_{\lambda^{\prime}}(x)$ means complex conjugation. This relation directly follows from the orthogonality of the exponential functions $e^{2 \pi \mathrm{i}\langle\mu, x\rangle}$ (entering into the definition of $E$-orbit functions) for different weights $\mu$ and from the fact that a given element $\nu \in P$ belongs to precisely one $E$-orbit function. In (5.10), $\left|\overline{F_{e}}\right|$ means an area of the domain $\overline{F_{e}}$.

Sometimes, it is difficult to find the area $\left|\overline{F_{e}}\right|$. In this case it is useful the following form of the formula (5.10):

$$
\int_{\mathrm{T}} E_{\lambda}(x) \overline{E_{\lambda^{\prime}}(x)} d x=\left|W_{e}\right| \delta_{\lambda \lambda^{\prime}}
$$

where $\mathrm{T}$ is the torus in $E_{n}$ described in Subsection 9.1 of [8]. If to assume that an area of $\mathrm{T}$ is equal to $1,|\mathrm{~T}|=1$, then $\left|\overline{F_{e}}\right|=\left|W_{e}\right|^{-1}$ and formula (5.10) takes the form

$$
\int_{\overline{F_{e}}} E_{\lambda}(x) \overline{E_{\lambda^{\prime}}(x)} d x=\delta_{\lambda \lambda^{\prime}}
$$


If $\lambda$ is an integral point which lies on a wall of the even dominant Weyl chamber $D_{+}^{e}$, then instead of (5.11) we have the relation

$$
\int_{\overline{F_{e}}} E_{\lambda}(x) \overline{E_{\lambda^{\prime}}(x)} d x=\left|W_{\lambda}\right|^{-1} \delta_{\lambda \lambda^{\prime}}
$$

where $W_{\lambda}$ is the subgroup of $W_{e}$ consisting of elements $w \in W_{e}$ such that $w \lambda=\lambda$.

\subsection{Solutions of the Laplace equation: the cases $A_{n}, B_{n}, C_{n}$ and $D_{n}$}

We use orthogonal coordinates $x_{1}, x_{2}, \ldots, x_{n+1}$ in the case of $A_{n}$ and the orthogonal coordinates $x_{1}, x_{2}, \ldots, x_{n}$ in the cases $B_{n}, C_{n}$ and $D_{n}$ (see section 3 ). The Laplace operator on $E_{r}$ in the orthogonal coordinates has the form

$$
\Delta=\frac{\partial^{2}}{\partial x_{1}^{2}}+\frac{\partial^{2}}{\partial x_{2}^{2}}+\cdots+\frac{\partial^{2}}{\partial x_{r}^{2}},
$$

where $r=n+1$ for $A_{n}$ and $r=n$ for $B_{n}, C_{n}$ and $D_{n}$. Let us consider the case $B_{n}$. We take a summand from the expression (4.26) for the $E$-orbit function $E_{\lambda}(x)$ of $B_{n}$ and act upon it by the operator $\Delta$. We get

$$
\begin{aligned}
\Delta e^{2 \pi \mathrm{i}\left((w(\varepsilon \lambda))_{1} x_{1}+\cdots+(w(\varepsilon \lambda))_{n} x_{n}\right)} \\
\quad=-4 \pi^{2}\left[\left(\varepsilon_{1} m_{1}\right)^{2}+\cdots+\left(\varepsilon_{n} m_{n}\right)^{2}\right] e^{2 \pi \mathrm{i}\left((w(\varepsilon \lambda))_{1} x_{1}+\cdots+(w(\varepsilon \lambda))_{n} x_{n}\right)} \\
\quad=-4 \pi^{2}\left(m_{1}^{2}+\cdots+m_{n}^{2}\right) e^{2 \pi \mathrm{i}\left((w(\varepsilon \lambda))_{1} x_{1}+\cdots+(w(\varepsilon \lambda))_{n} x_{n}\right)} \\
\quad=-4 \pi^{2}\langle\lambda, \lambda\rangle e^{2 \pi \mathrm{i}\left((w(\varepsilon \lambda))_{1} x_{1}+\cdots+(w(\varepsilon \lambda))_{n} x_{n}\right)}
\end{aligned}
$$

where $\lambda=\left(m_{1}, m_{2}, \ldots, m_{n}\right)$ is the element of $D_{+}^{e}\left(B_{n}\right)$, determining $E_{\lambda}(x)$, in the orthogonal coordinates and $w \in S_{n}^{e} \equiv S_{n} / S_{2}$. Since this action of $\Delta$ does not depend on a summand from (4.26), we have

$$
\Delta E_{\lambda}(x)=-4 \pi^{2}\langle\lambda, \lambda\rangle E_{\lambda}(x) .
$$

For $A_{n}, C_{n}$ and $D_{n}$ this formula also holds and the corresponding proofs are the same. Remark that in the case $A_{n}$ the scalar product $\langle\lambda, \lambda\rangle$ is equal to

$$
\langle\lambda, \lambda\rangle=m_{1}^{2}+m_{2}^{2}+\cdots+m_{n+1}^{2} .
$$

Thus, $E$-orbit functions are eigenfunctions of the Laplace operator on the Euclidean space $E_{r}$.

\subsection{The Laplace operator in $\omega$-basis}

We may parametrize points of $E-n$ by coordinates in the $\omega$-basis: $x=\theta_{1} \omega_{1}+\cdots+\theta_{n} \omega_{n}$. Denoting by $\partial_{k}$ partial derivative with respect to $\theta_{k}$, we have the Laplace operator $\Delta$ in the form

$$
\Delta=\frac{1}{2} \sum_{i, j=1}^{n}\left\langle\alpha_{j}, \alpha_{j}\right\rangle^{-1} M_{i j} \partial_{i} \partial_{j},
$$

where $\left(M_{i j}\right)$ is the corresponding Cartan matrix. One can see that it is indeed the Laplace operator as follows. The matrix $\left(S_{i j}\right)=\left(\left\langle\alpha_{j}, \alpha_{j}\right\rangle^{-1} M_{i j}\right)$ is symmetric with respect to transposition and its determinant is positive. Hence it can be diagonalized, so that $\Delta$ becomes a sum of second derivatives with no mixed derivative terms. 
We write down explicit form of the Laplace operators in coordinates in the $\omega$-basis for ranks 2 and 3. For rank two the operator $\Delta$ is of the form

$$
\begin{array}{ll}
A_{2}: & \left(\partial_{1}^{2}-\partial_{1} \partial_{2}+\partial_{2}^{2}\right) E_{\lambda}(x)=-\frac{4 \pi^{2}}{3}\left(a^{2}+a b+b^{2}\right) E_{\lambda}(x), \\
C_{2}: & \left(2 \partial_{1}^{2}-2 \partial_{1} \partial_{2}+\partial_{2}^{2}\right) E_{\lambda}(x)=-2 \pi^{2}\left(a^{2}+4 a b+4 b^{2}\right) E_{\lambda}(x), \\
G_{2}: & \left(\partial_{1}^{2}-3 \partial_{1} \partial_{2}+3 \partial_{2}^{2}\right) E_{\lambda}(x)=-\frac{4 \pi^{2}}{3}\left(3 a^{2}+3 a b+b^{2}\right) E_{\lambda}(x) .
\end{array}
$$

Here, $\lambda=(a b)$ and $x=\left(\theta_{1} \theta_{2}\right)$.

In the semisimple case $A_{1} \times A_{1}$ one has $M=2\left(\begin{array}{ll}1 & 0 \\ 0 & 1\end{array}\right)$, therefore $\Delta=2 \partial_{1}^{2}+2 \partial_{2}^{2}$, and $E_{\lambda}(x)$ is the product of two $E$-orbit functions, one from each $A_{1}$.

There are three 3 -dimensional cases, namely $A_{3}, B_{3}$, and $C_{3}$. For $A_{3}, B_{3}$, and $C_{3}$ the result can be represented by the formulas

$$
\begin{array}{ll}
A_{3}: & \Delta=\partial_{1}^{2}+\partial_{2}^{2}+\partial_{3}^{2}-\partial_{1} \partial_{2}-\partial_{2} \partial_{3}, \\
B_{3}: & \Delta=\partial_{1}^{2}+\partial_{2}^{2}+2 \partial_{3}^{2}-\partial_{1} \partial_{2}-2 \partial_{2} \partial_{3}, \\
C_{3}: & \Delta=2 \partial_{1}^{2}+2 \partial_{2}^{2}+2 \partial_{3}^{2}-2 \partial_{1} \partial_{2}-2 \partial_{2} \partial_{3} .
\end{array}
$$

In all these case we have

$$
\Delta E_{\lambda}(x)=-4 \pi^{2}\langle\lambda, \lambda\rangle E_{\lambda}(x) .
$$

\section{$5.10 E$-orbit functions as eigenfunctions of other operators}

$E$-orbit functions are eigenfunctions of many other operators. Let us consider examples of such operators.

With each $y \in E_{n}$ we associate the shift operator $T_{y}$ which acts on the exponential functions $e^{2 \pi \mathrm{i}\langle\lambda, x\rangle}$ as

$$
T_{y} e^{2 \pi \mathrm{i}\langle\lambda, x\rangle}=e^{2 \pi \mathrm{i}\langle\lambda, x+y\rangle}=e^{2 \pi \mathrm{i}\langle\lambda, y\rangle} e^{2 \pi \mathrm{i}\langle\lambda, x\rangle} .
$$

An action of elements of the even Weyl group $W_{e}$ on functions, given on $E_{n}$, is given as $w f(x)=$ $f(w x)$. For each $y \in E_{n}$ we define an operator acting on orbit functions by the formula

$$
D_{y}=\sum_{w \in W_{e}}(\operatorname{det} w) w T_{y} .
$$

Then

$$
\begin{aligned}
D_{y} \hat{E}_{\lambda}(x) & =D_{y} \sum_{w \in W} e^{2 \pi \mathrm{i}\langle w \lambda, x\rangle}=\sum_{w^{\prime} \in W_{e}} \sum_{w \in W_{e}} e^{2 \pi \mathrm{i}\langle w \lambda, y\rangle} e^{2 \pi \mathrm{i}\left\langle w \lambda, w^{\prime} x\right\rangle} \\
& =\sum_{w \in W_{e}} e^{2 \pi \mathrm{i}\langle w \lambda, y\rangle} \sum_{w^{\prime} \in W_{e}} e^{2 \pi \mathrm{i}\left\langle w \lambda, w^{\prime} x\right\rangle}=\sum_{w \in W_{e}} e^{2 \pi \mathrm{i}\langle w \lambda, y\rangle} \sum_{w^{\prime} \in W_{e}} e^{2 \pi \mathrm{i}\left\langle w^{\prime-1} w \lambda, x\right\rangle} \\
& =\sum_{w \in W_{e}} e^{2 \pi \mathrm{i}\langle w \lambda, y\rangle} \hat{E}_{\lambda}(x)=\hat{E}_{\lambda}(y) \hat{E}_{\lambda}(x),
\end{aligned}
$$

that is, $\hat{E}_{\lambda}(x)$ (and therefore $E_{\lambda}(x)$ ) is an eigenfunction of the operator $D_{y}$ with eigenvalue $\hat{E}_{\lambda}(y)$.

It is shown similarly that in the cases of $A_{n}, B_{n}, C_{n}, D_{n}$ the functions $E_{\lambda}(x)$ are eigenfunctions of the operators

$$
\sum_{w \in W_{e}} w \frac{\partial^{2}}{\partial x_{i}^{2}}, \quad i=1,2, \ldots, r
$$

where $x_{1}, x_{2}, \ldots, x_{r}$ are orthogonal coordinates of the point $x, r=n+1$ for $A_{n}$ and $r=n$ for other cases. In fact, these operators are multiple to the Laplace operator $\Delta$. 


\section{Decomposition of products of $E$-orbit functions}

In this section we show how to decompose products of $E$-orbit functions into sums of $E$-orbit functions. Such operations are fulfilled by means of the corresponding decompositions of $W_{e^{-}}$ orbits.

\subsection{Products of orbit functions}

Invariance of $E$-orbit functions $E_{\lambda}$ with respect to the even Weyl group $W_{e}$ leads to the following statement:

Proposition 1. A product of E-orbit functions expands into a sum of $W_{e}$-orbit functions:

$$
E_{\lambda} E_{\mu}=\sum_{\nu} n_{\nu} E_{\nu}
$$

where $n_{\nu}$ are non-negative integers, which shows how many times the orbit function $E_{\nu}$ is contained in the product $E_{\lambda} E_{\mu}$.

Proof. For $w \in W_{e}$ we have

$$
E_{\lambda}(w x) E_{\mu}(w x)=E_{\lambda}(x) E_{\mu}(x) .
$$

Therefore, the product $E_{\lambda}(x) E_{\mu}(x)$ is a finite sum of exponential functions, which is invariant with respect to $W_{e}$. Hence, it can be expanded into $E$-orbit functions. Representing the product $E_{\lambda}(x) E_{\mu}(x)$ as a sum of exponential functions we see that these exponential functions enter into this sum with positive integral coefficients. This means that coefficients $n_{\nu}$ in (6.1) are nonnegative integers. The proposition is proved.

Under termwise multiplication of $E$-orbit functions $E_{\lambda}(x)$ and $E_{\mu}(x)$ we multiply exponential functions,

$$
e^{2 \pi \mathrm{i}\langle\nu, x\rangle} e^{2 \pi \mathrm{i}\left\langle\nu^{\prime}, x\right\rangle}=e^{2 \pi \mathrm{i}\left\langle\nu+\nu^{\prime}, x\right\rangle} .
$$

This reduces the procedure of multiplication of $E$-orbit functions $E_{\lambda}(x)$ and $E_{\mu}(x)$ to the procedure of multiplication of the corresponding $W_{e}$-orbits $O_{e}(\lambda)$ and $O_{e}(\mu)$. A product of these orbits is defined as follows.

A product $O_{e}(\lambda) \otimes O_{e}\left(\lambda^{\prime}\right)$ of two $W_{e}$-orbits $O_{e}(\lambda)$ and $O_{e}\left(\lambda^{\prime}\right)$ is the set of all points of the form $\lambda_{1}+\lambda_{2}$, where $\lambda_{1} \in O_{e}(\lambda)$ and $\lambda_{2} \in O_{e}\left(\lambda^{\prime}\right)$. Since a set of points $\lambda_{1}+\lambda_{2}, \lambda_{1} \in O_{e}(\lambda), \lambda_{2} \in O_{e}\left(\lambda^{\prime}\right)$,

is invariant with respect to action of the corresponding even Weyl group $W_{e}$, each product of $W_{e}$-orbits is decomposable into a sum of $W_{e}$-orbits. If $\lambda=0$, then $O_{e}(\lambda) \otimes O_{e}\left(\lambda^{\prime}\right)=O_{e}\left(\lambda^{\prime}\right)$. If $\lambda^{\prime}=0$, then $O_{e}(\lambda) \otimes O_{e}\left(\lambda^{\prime}\right)=O_{e}(\lambda)$. In what follows we assume that $\lambda \neq 0$ and $\lambda^{\prime} \neq 0$. Decomposition of products of two $W_{e^{-}}$orbits into separate $W_{e^{-}}$orbits is not a simple task.

\subsection{Decomposition of products of $W_{e}$-orbits}

If $O_{e}(\lambda)$ and $O_{e}\left(\lambda^{\prime}\right)$ are two $W_{e}$-orbits such that $\lambda$ and $\lambda^{\prime}$ are dominant and lie on walls of the dominant Weyl chamber, then these orbits in fact coincide with the corresponding $W$-orbits $O(\lambda)$ and $O\left(\lambda^{\prime}\right)$, respectively. In this case we can apply to this product a procedure of decomposition of a product of $W$-orbits (see Section 4 in [7]). Decomposing this product into $W$-orbits, we make further the following. If a resulting $W$-orbit $O(\mu)$ is such that $\mu$ lies on a wall of the dominant Weyl chamber, then $O(\mu)$ is $W_{e}$-orbit, and $O_{e}(\mu)$ is contained in the product $O_{e}(\lambda) \otimes O_{e}\left(\lambda^{\prime}\right)$ of $W_{e}$-orbits with a multiplicity equal to a multiplicity of $W$-orbit $O(\mu)$ in the product $O(\mu) \otimes O\left(\mu^{\prime}\right)$. If a resulting $W$-orbit $O(\mu)$ is such that $\mu$ does not lie on a wall of the dominant Weyl chamber, 
then this $W$-orbit consists of two $W_{e}$-orbit $O_{e}(\mu)$ and $O\left(r_{\alpha} \mu\right)$. Moreover, multiplicities of these $W_{e}$-orbits are determined as in the previous case. Thus, in our case a decomposition of $W_{e^{-o r b i t s}}$ is completely determined by a decomposition of the corresponding $W$-orbits.

Let now the $W_{e}$-orbits $O_{e}(\lambda)$ and $O_{e}\left(\lambda^{\prime}\right)$ be such that $\lambda$ is on a wall of the dominant Weyl chamber and $\lambda^{\prime}$ is a strictly dominant element. Then $O_{e}(\lambda)$ can be considered as $W$-orbit $O(\lambda)$. Instead of the $W_{e}$-orbit $O_{e}\left(\lambda^{\prime}\right)$ we consider the signed $W$-orbit $O^{ \pm}\left(\lambda^{\prime}\right)$. (This signed orbit consists of two $W_{e}$-orbits $O_{e}(\lambda)$ and $O_{e}\left(r_{\alpha} \lambda\right)$, and points of $O_{e}\left(r_{\alpha} \lambda\right)$ are taken with the sign minus.) Our problem of decomposition of the product $O_{e}(\lambda) \otimes O_{e}\left(\lambda^{\prime}\right)$ is reduced to decomposition of the product $O(\lambda) \otimes O^{ \pm}\left(\lambda^{\prime}\right)$ of a $W$-orbit and a signed $W$-orbit. Decompositions of such products are studied in Section 7 in [8]. The product $O(\lambda) \otimes O^{ \pm}\left(\lambda^{\prime}\right)$ decomposes into signed orbits, which are taken with sign plus or sign minus, that is,

$$
O(\lambda) \otimes O^{ \pm}\left(\lambda^{\prime}\right)=\bigcup_{\mu} n_{\mu} O^{ \pm}(\mu)
$$

where $n_{\mu}$ are positive or negative integers. Now a result for decomposition of the product $O_{e}(\lambda) \otimes$ $O_{e}\left(\lambda^{\prime}\right)$ can be formulated as follows. If a sign orbit $O^{ \pm}(\mu)$ is contained in the decomposition of $O(\lambda) \otimes O^{ \pm}\left(\lambda^{\prime}\right)$ with positive coefficient $n_{\mu}$, then the product $O_{e}(\lambda) \otimes O_{e}\left(\lambda^{\prime}\right)$ contains the $W_{e}$-orbit $O_{e}(\mu)$ with multiplicity $n_{\mu}$. If an orbit $O^{ \pm}(\mu)$ is contained in decomposition of $O(\lambda) \otimes O^{ \pm}\left(\lambda^{\prime}\right)$ with negative coefficient $n_{\mu}$, then the product $O_{e}(\lambda) \otimes O_{e}\left(\lambda^{\prime}\right)$ contains the $W_{e}$-orbit $O_{e}\left(r_{\alpha} \mu\right)$ with multiplicity $-n_{\mu}$. This procedure determines in the decomposition of $O_{e}(\lambda) \otimes O_{e}\left(\lambda^{\prime}\right)$ all $W_{e}$-orbits $O_{e}(\mu)$ and $O_{e}\left(r_{\alpha} \mu\right)$ for which $\mu$ is strictly positive and does not give $W_{e}$-orbits $O_{e}(\mu)$ with $\mu$ lying on a wall of the dominant Weyl chamber.

In order to find in the decomposition $O_{e}(\lambda) \otimes O_{e}\left(\lambda^{\prime}\right)$ the $W_{e}$-orbits $O_{e}(\mu)$ with $\mu$ lying on a wall of the dominant Weyl chamber, along with the product $O(\lambda) \otimes O^{ \pm}\left(\lambda^{\prime}\right)$ we have to consider also the product $O(\lambda) \otimes O\left(\lambda^{\prime}\right)$ of $W$-orbit functions (which are not signed orbits).

Below we consider the case of products $O_{e}(\lambda) \otimes O_{e}\left(\lambda^{\prime}\right)$ of $W_{e}$-orbits, when $\lambda$ and $\lambda^{\prime}$ do not lie on walls of even dominant Weyl chamber. For simplicity we consider the case when $\lambda$ and $\lambda^{\prime}$ are strictly dominant.

Let $O_{e}(\lambda)=\left\{w \lambda \mid w \in W_{e}\right\}$ and $O_{e}(\mu)=\left\{w \mu \mid w \in W_{e}\right\}$ be two $W_{e}$-orbits with strictly dominant $\lambda$ and $\mu$. Then

$$
\begin{aligned}
& O_{e}(\lambda) \otimes O_{e}(\mu)=\left\{w \lambda+w^{\prime} \mu \mid w, w^{\prime} \in W_{e}\right\} \\
& \quad=\left\{w \lambda+w_{1} \mu \mid w \in W_{e}\right\} \cup\left\{w \lambda+w_{2} \mu \mid w \in W_{e}\right\} \cup \cdots \cup\left\{w \lambda+w_{s} \mu \mid w \in W_{e}\right\},
\end{aligned}
$$

where $w_{1}, w_{2}, \ldots, w_{s}$ is the set of elements of $W_{e}$. Since a product of $W_{e}$-orbits is invariant with respect to $W_{e}$, for decomposition of the product $O_{e}(\lambda) \otimes O_{e}(\mu)$ into separate $W_{e}$-orbits it is necessary to separate from each term of the right hand side of (6.2) elements, which belong to the even Weyl chamber $D_{+}^{e}$. That is, $O_{e}(\lambda) \otimes O_{e}(\mu)$ is a union of the $W_{e^{-}}$orbits, determined by points from

$$
D_{e}\left(\left\{w \lambda+w_{1} \mu \mid w \in W_{e}\right\}\right), D\left(\left\{w \lambda+w_{2} \mu \mid w \in W_{e}\right\}\right), \ldots, D_{e}\left(\left\{w \lambda+w_{s} \mu \mid w \in W_{e}\right\}\right),
$$

where $D_{e}\left(\left\{w \lambda+w_{i} \mu \mid w \in W_{e}\right\}\right)$ means the set of elements in the collection $\left\{w \lambda+w_{i} \mu \mid w \in W_{e}\right\}$ belonging to $D_{+}^{e}$.

Proposition 2. Let $\lambda$ and $\mu$ be elements of $D_{+}^{e}$, which do not lie on walls of $D_{+}^{e}$. The product $O_{e}(\lambda) \otimes O_{e}(\mu)$ consists only of $W_{e}$-orbits of the form $O_{e}(|w \lambda+\mu|), w \in W_{e}$, where $|w \lambda+\mu|$ is an element of $D_{+}^{e}$ in the $W_{e}$-orbit containing $w \lambda+\mu$. Moreover, each such $W_{e}$-orbit $O_{e}(|w \lambda+\mu|)$, $w \in W_{e}$, belongs to the product $O_{e}(\lambda) \otimes O_{e}(\mu)$.

Proof. For each dominant element $w \lambda+w_{i} \mu$ from (6.3) there exists an element $w^{\prime \prime} \in W_{e}$ such that $w^{\prime \prime}\left(w \lambda+w_{i} \mu\right)=w^{\prime} \lambda+\mu$. It means that $w \lambda+w_{i} \mu$ is of the form $\left|w^{\prime} \lambda+\mu\right|, w^{\prime} \in W_{e}$. 
Conversely, take any element $w \lambda+\mu, w \in W_{e}$. It belongs to the product $O_{e}(\lambda) \otimes O_{e}(\mu)$. This means that $|w \lambda+\mu|$ also belongs to this product. Therefore, the orbit $O_{e}(|w \lambda+\mu|)$ is contained in $O_{e}(\lambda) \otimes O_{e}(\mu)$. Proposition is proved.

It follows from Proposition 2 that for decomposition of the product $O_{e}(\lambda) \otimes O_{e}(\mu)$ into separate orbits we have to take all elements $w \lambda+\mu, w \in W_{e}$, and to find the corresponding dominant elements $|w \lambda+\mu|, w \in W_{e}$.

For $\lambda$ and $\mu$ from Proposition 2 the product $O_{e}(\lambda) \otimes O_{e}(\mu)$ contains $W_{e}$-orbits with multiplicities 1 , that is,

$$
O_{e}(\lambda) \otimes O_{e}(\mu)=\bigcup_{w \in W_{e}} O_{e}(|w \lambda+\mu|)
$$

If $\lambda$ and $\mu$ lie on walls of $D_{+}^{e}$, then some orbits can be contained in the decomposition of $O_{e}(\lambda) \otimes O_{e}(\mu)$ with a multiplicity. The most difficult problem under consideration of products of orbits is to find these multiplicities.

Formulas (6.2) and (6.3) are related to decompositions of products $O_{e}(\lambda) \otimes O_{e}(\mu)$, when $\lambda$ and $\mu$ do not lie on walls of the domain $D_{+}^{e}$. Now we assume that $\lambda$ or/and $\mu$ may lie on walls of $D_{+}^{e}$. Then formula (6.2) is replaced by

$$
\begin{aligned}
& O_{e}(\lambda) \otimes O_{e}(\mu)=\left\{w \lambda+w^{\prime} \mu \mid w \in W_{e} / W_{\lambda}, w^{\prime} \in W_{e} / W_{\mu}\right\} \\
& \quad=\left\{w+w_{1} \mu \mid w \in W_{e} / W_{\lambda}\right\} \cup\left\{w \lambda+w_{2} \mu \mid w \in W_{e} / W_{\lambda}\right\} \cup \cdots \cup\left\{w \lambda+w_{r} \mu \mid w \in W_{e} / W_{\lambda}\right\}
\end{aligned}
$$

where $W_{\lambda}$ is the subgroup of $W_{e}$ consisting of elements leaving $\lambda$ invariant and $w_{1}, w_{2}, \ldots, w_{r}$ is the set of elements of $W_{e} / W_{\mu}$. In this case, $O_{e}(\lambda) \otimes O_{e}(\mu)$ is a union of the $W_{e}$-orbits, determined by points from

$$
\begin{gathered}
D_{e}\left(\left\{w \lambda+w_{1} \mu \mid w \in W_{e} / W_{\lambda}\right\}\right), D\left(\left\{w \lambda+w_{2} \mu \mid w \in W_{e} / W_{\lambda}\right\}\right), \ldots, \\
D_{e}\left(\left\{w \lambda+w_{r} \mu \mid w \in W_{e} / W_{\lambda}\right\}\right),
\end{gathered}
$$

where $D_{e}\left(\left\{w \lambda+w_{i} \mu \mid w \in W_{e}\right\}\right)$ has the same sense as in (6.3).

Proposition 3. Let $O_{e}(\lambda)$ and $O_{e}(\mu), \lambda, \mu \in D_{+}^{e}$, be $W_{e}$-orbits such that $\lambda \neq 0$ and $\mu \neq 0$, and let elements $w \lambda+\mu, w \in W_{e} / W_{\lambda}$, belong to $D_{e}^{+}$and do not belong to walls of even Weyl dominant chamber $D_{+}^{e}$. Then

$$
O_{e}(\lambda) \otimes O_{e}(\mu)=\bigcup_{w \in W_{e} / W_{\lambda}} O_{e}(w \lambda+\mu)
$$

Proof. Under the conditions of the proposition the set of elements $w \lambda+\mu, w \in W_{e} / W_{\lambda}$, is contained in the first set of (6.6) if $w_{1}$ coincides with the identical transformation. Moreover, $W_{w \lambda+\mu} \equiv\left\{w^{\prime} \in W_{e} ; w^{\prime}(w \lambda+\mu)=w \lambda+\mu\right\}=\{1\}$ for all elements $w \in W_{e}$. Then $\mu$ does not lies on the boundary of $D_{e}^{+}$, that is, $W_{\mu}=\{1\}$. Let us show that the collection (6.6) contains only one non-empty set $D\left(\left\{w \lambda+\mu \mid w \in W_{e} / W_{\lambda}\right\}\right)$. Indeed, let $w \lambda+w_{i} \mu, w_{i} \neq 1$, belongs to $D_{e}^{+}$. Then

$$
w_{i}^{-1}\left(w \lambda+w_{i} \mu\right)=w_{i}^{-1} w \lambda+\mu \in D_{+}^{e} .
$$

Since $W_{w_{i}^{-1} w \lambda+\mu}=\{1\}$, then $w_{i}^{-1}\left(w \lambda+w_{i} \mu\right)$ is an intrinsic point of $D_{+}^{e}$. Therefore, $w \lambda+w_{i} \mu \notin$ $D_{+}^{e}$. This contradicts to the condition that $w \lambda+w_{i} \mu \in D_{+}^{e}$ and, therefore, the collection (6.6) contains only one non-empty set. The set of orbits, corresponding to the points of $D(\{w \lambda+\mu \mid w \in$ $\left.W_{e} / W_{\lambda}\right\}$ ), coincides with the right hand side of (6.7). Proposition is proved. 
Proposition 4. If $\lambda, \mu \in D_{+}^{e}$, then $O_{e}(\lambda) \otimes O_{e}(\mu)$ consists only of $W_{e}$-orbits of the form $O_{e}(|w \lambda+\mu|), w \in W_{e} / W_{\lambda}$, where $|w \lambda+\mu|$ is an element of $D_{+}^{e}$ in the $W_{e^{-o r b i t}}$ containing $w \lambda+\mu$. Moreover, each such $W_{e}$-orbit $O_{e}(|w \lambda+\mu|)$ belongs to the product $O_{e}(\lambda) \otimes O_{e}(\mu)$.

Proof is similar to that of Proposition 2 and we omit it.

Under conditions of Proposition 4 the relation (6.4) in general is not true. The simplest counterexample is when $\mu=0$. Then according to this formula $O_{e}(\lambda) \otimes O_{e}(\mu)=O_{e}(\lambda) \cup O_{e}(\lambda) \cup$ $\cdots \cup O_{e}(\lambda)\left(\mid W_{e} / W_{\lambda}\right.$ times $)$. However, as we know, $O_{e}(\lambda) \otimes O_{e}(\mu)=O_{e}(\lambda)$.

Proposition 3 states that instead of (6.4) we have

$$
O_{e}(\lambda) \otimes O_{e}(\mu) \subseteq \bigcup_{w \in W_{e}} O_{e}(|w \lambda+\mu|)
$$

Note that some orbits on the right hand side can coincide.

Proposition 5. Let $O_{e}(\mu)$ and $O_{e}(\mu)$ be $W_{e}$-orbits such that $\lambda \neq 0$ and $\mu \neq 0$, and let all elements $w \lambda+\mu, w \in W_{e}$, belong to $D_{+}^{e}$. Then

$$
O_{e}(\lambda) \otimes O_{e}(\mu)=\bigcup_{w \in W / W_{\lambda}} n_{w \lambda+\mu} O_{e}(w \lambda+\mu)
$$

where $n_{w \lambda+\mu}=\left|W_{w \lambda+\mu}\right|$.

Proof. Since $\lambda \neq 0$, all elements $w \lambda+\mu, w \in W_{e} / W_{\lambda}$, belong to $D_{e}^{+}$if and only if $W_{\mu}=\{1\}$. Then on the right hand side of (6.5) there are $\left|W_{e}\right|$ terms. If the element $w \lambda+\mu$ belongs to $D_{e}^{+}$ and does not lie on a wall, that is, $W_{w \lambda+\mu}=\{1\}$, then this element is met only in one term. This means that the multiplicity $n_{w \lambda+\mu}$ of $O_{e}(w \lambda+\mu)$ in the product $O_{e}(\lambda) \otimes O_{e}(\mu)$ is 1 . If $w \lambda+\mu$ is placed on some wall of $D_{+}^{e}$, then it is met in $n_{w \lambda+\mu}=\left|W_{w \lambda+\mu}\right|$ terms (since the elements $w^{\prime} w \lambda+w^{\prime} \mu, w^{\prime} \in W_{w \lambda+\mu}$, belong to pairwise different terms in (6.5)). Therefore, there are $n_{w \lambda+\mu}$ orbits $O_{e}(w \lambda+\mu)$ in the decomposition of $O_{e}(\lambda) \otimes O_{e}(\mu)$. Proposition is proved.

Proposition 6. If $W_{\mu}=\{1\}$ and none of the points $w \lambda+\mu, w \in W_{e} / W_{\lambda}$, lies on a wall of some even Weyl chamber, then

$$
O_{e}(\lambda) \otimes O_{e}(\mu)=\bigcup_{w \in W_{e} / W_{\lambda}} O_{e}(|w \lambda+\mu|)
$$

Proof. For the product $O_{e}(\lambda) \times O_{e}(\mu)$ the inclusion

$$
O_{e}(\lambda) \otimes O_{e}(\mu) \subseteq \bigcup_{w \in W_{e}} O_{e}(|w \lambda+\mu|)
$$

holds. Each orbit $O_{e}(|w \lambda+\mu|), w \in W_{e} / W_{\lambda}$, has $\left|W_{e}\right|$ elements and is contained in $O_{e}(\lambda) \otimes$ $O_{e}(\mu)$. Therefore, numbers of elements in both sides of (6.9) coincide. This means that the inclusion (6.9) is in fact an equality. Proposition is proved.

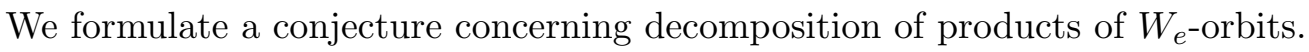

Conjecture. Let $O_{e}(\lambda)$ and $O_{e}(\mu), \lambda \neq 0, \mu \neq 0$, be orbits, and let $\mu$ belong to $D_{e}^{+}$and do not lie on a wall of $D_{+}^{e}$. If for some $w \in W_{e}$ the element $w \lambda+\mu$ belongs to $D_{e}^{+}$and does not lie on a wall, then a multiplicity of $O_{e}(w \lambda+\mu)$ in $O_{e}(\lambda) \otimes O_{e}(\mu)$ is 1 .

At the end of this subsection we formulate a method for decomposition of products $O_{e}(\lambda) \otimes$ $O_{e}(\mu)$, which follows from the statement of Proposition 4. On the first step we shift all points of the orbit $O_{e}(\lambda)$ by $\mu$. As a result, we obtain the set of points $w \lambda+\mu, w \in W_{e}$. On the 
second step, we map elements of this set, which do not belong to $D_{e}^{+}$, by elements of the even Weyl group $W_{e}$ to the chamber $D_{e}^{+}$. On this step we obtain the set $|w \lambda+\mu|, w \in W_{e}$. Then by Proposition $4, O_{e}(\lambda) \otimes O_{e}(\mu)$ consists of the orbits $O_{e}(|w \lambda+\mu|)$. On the third step, we determine multiplicities of these orbits, taking into account the above propositions or making direct calculations.

\subsection{Decomposition of products for $A_{2}$}

We give examples of decompositions of products of $W_{e}$-orbits for the cases $A_{2}$ and $C_{2}$. Orbits for these cases are placed on a plane. Therefore, decompositions can be done by geometrical calculations on this plane. These cases can be easily considered also by using for orbit points orthogonal coordinates from Section 3. The corresponding even Weyl groups have a simple description in these coordinates and this gives a possibility to make calculations in a simple manner.

For the case of $A_{2}$ at $a \neq b, a>0, b>0$, and at $c>0$ we have

$$
\begin{aligned}
A_{2}: & O_{e}(a b) \otimes O_{e}(c 0) & =O_{e}(a+c b) \cup O_{e}(a-c b+c) & \\
& \cup O_{e}(-a-b+c a) & & (a>c>b), \\
O_{e}(a b) \otimes O_{e}(c 0) & =O_{e}(a+c b) \cup O_{e}(a-c b+c) \cup O_{e}(a b-c) & & (a>c, b>c), \\
O_{e}(a b) \otimes O_{e}(c 0) & \left.=O_{e}(a+c b) \cup O(-a-b+c a)\right) \cup O_{e}(0 a+b) & & (a=c>b), \\
O_{e}(a b) \otimes O_{e}(c 0) & =O_{e}(a+c b) \cup O_{e}(a b-c) \cup O_{e}(0 a+b) & & (b>a=c), \\
O_{e}(a b) \otimes O_{e}(c 0) & =O_{e}(a+c b) \cup O_{e}(a-c b+c) \cup O_{e}(a 0) & & (a<b=c), \\
O_{e}(a b) \otimes O_{e}(c 0) & =O_{e}(a+c b) \cup O_{e}(a-c b+c) & & \\
& \cup O_{e}(c-a-b a) & & (c>a+b), \\
O_{e}(a b) \otimes O_{e}(c 0) & =O_{e}(a+c b) \cup O^{ \pm}(a-c b+c) & & (a+b>c>b), \\
& \cup O^{ \pm}(-a-b+c b) & & (a+b>b>c),
\end{aligned}
$$

$$
\begin{aligned}
& A_{2}: \quad O_{e}(-a a+b) \otimes O_{e}(c 0)=O_{e}(-a-c a+b+c) \cup O_{e}(c-a a+b) \\
& \cup O_{e}(a+b-c c-b) \quad(a>c>b), \\
& O_{e}(-a a+b) \otimes O_{e}(c 0)=O_{e}(-a-c a+b+c) \cup O_{e}(c-a a+b) \\
& \cup O_{e}(-a a+b-c) \quad(a>c, b>c), \\
& \left.O_{e}(-a a+b) \otimes O_{e}(c 0)=O_{e}(-a-c a+b+c) \cup O_{e}(a+b-c c-b)\right) \\
& \cup O_{e}(0 a+b) \quad(a=c>b), \\
& O_{e}(-a a+b) \otimes O_{e}(c 0)=O_{e}(-a-c a+b+c) \cup O_{e}(-a a+b-c) \\
& \cup O_{e}(0 a+b) \quad(b>a=c), \\
& O_{e}(-a a+b) \otimes O_{e}(c 0)=O_{e}(-a-c a+b+c) \cup O_{e}(c-a a+b) \\
& \cup O_{e}(a 0) \quad(a<b=c), \\
& O_{e}(-a a+b) \otimes O_{e}(c 0)=O_{e}(-a-c a+b+c) \cup O_{e}(c-a a+b) \\
& \cup O_{e}(a+b-c c-b) \quad(c>a+b), \\
& O_{e}(-a a+b) \otimes O_{e}(c 0)=O_{e}(-a-c a+b+c) \cup O_{e}(c-a a+b) \\
& \cup O_{e}(a+b-c c-a) \quad(a+b>c>b), \\
& O_{e}(-a a+b) \otimes O(c 0)=O_{e}(-a-c a+b+c) \cup O_{e}(c-a a+b) \\
& \cup O_{e}(-a a+b-c) \\
& (a+b>b>c) .
\end{aligned}
$$


Note that $(-a a+b)$ belongs to $D_{+}^{e}$ and does not belong to $D_{+}$. If $a=b$, then we get

$$
\begin{aligned}
& O_{e}(a a) \otimes O_{e}(c 0)=O_{e}(a+c a) \cup O_{e}(c-2 a a) \cup O_{e}(a-c a+c) \quad(c>2 a), \\
& O_{e}(a a) \otimes O_{e}(c 0)=O_{e}(a+c a) \cup O_{e}(c-2 a a) \cup O_{e}(a-c a+c) \quad(2 a>c>a), \\
& O_{e}(a a) \otimes O_{e}(c 0)=O_{e}(a+c a) \cup O_{e}(a-c a+c) \cup O_{e}(a a-c) \quad(a>c) .
\end{aligned}
$$

The $W_{e}$-orbit $O_{e}\left(r_{\alpha}(a a)\right)$ has the form $O_{e}(-a 2 a)$. The products of such orbits with the $W_{e}$-orbit $O_{e}(c 0)$ decompose into $W_{e^{-o r b i t s}}$ as

$$
\begin{aligned}
O_{e}(-a 2 a) \otimes O_{e}(c 0) & =O_{e}(-a-c 2 a+c) \cup O_{e}(2 a-c c-a) & \\
& \cup O_{e}(c-a 2 a) & (c>2 a), \\
O_{e}(-a 2 a) \otimes O_{e}(c 0) & O_{e}(-a-c 2 a+c) \cup O_{e}(2 a-c c-a) & \\
& \cup O_{e}(c-a 2 a) & (2 a>c>a), \\
O_{e}(-a 2 a) \otimes O_{e}(c 0) & O_{e}(-a-c 2 a+c) \cup O_{e}(c-a 2 a) & \\
& \cup O_{e}(-a 2 a-c) & (a>c) .
\end{aligned}
$$

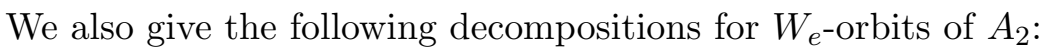

$$
\begin{aligned}
& A_{2}: \quad O_{e}(a 0) \otimes O_{e}(b 0)=O_{e}(a+b 0) \cup O_{e}(-a+b a) \cup O_{e}(a-b b) \quad(a<b), \\
& O_{e}(a 0) \otimes O_{e}(a 0)=O_{e}(2 a 0) \cup 2 O_{e}(0 a), \\
& O_{e}(a 0) \otimes O_{e}(0 b)=O_{e}(a b) \cup O_{e}(-a a+b) \cup O_{e}(0-a+b) \quad(a<b) \text {, } \\
& O_{e}(a 0) \otimes O_{e}(0 a)=O_{e}(a a) \cup O_{e}(-a 2 a) \cup 3 O_{e}\left(\begin{array}{ll}
0 & 0
\end{array}\right) \text {. }
\end{aligned}
$$

\subsection{Decomposition of products for $C_{2}$}

For dominant elements $(a b)$ products $W_{e}$-orbits for $C_{2}$ are of the form

$$
\begin{aligned}
& C_{2}: \quad O_{e}(a b) \otimes O_{e}(c 0)=O_{e}(a+c b) \cup O_{e}(a+2 b-c-a-b+c) \cup O_{e}(a-c b+c) \\
& \cup O_{e}(c-2 b-a a+b) \quad(a+b-c<b), \\
& O_{e}(a b) \otimes O_{e}(c 0)=O_{e}(a+c b) \cup O_{e}(a+2 b-c c-a-b) \cup O_{e}(a-c b+c) \\
& \cup O_{e}(c-a-2 b a+b) \quad(b>c-a-b, a>c), \\
& O_{e}(a b) \otimes O_{e}(c 0)=O_{e}(a+c b) \cup O_{e}(a+2 b-c c-a-b) \cup O_{e}(a-c b+c) \\
& \cup O_{e}(c-a-2 b a+b) \quad(b>c-a-b, c>a), \\
& O_{e}(a b) \otimes O_{e}(c 0)=O_{e}(a+c b) \cup O_{e}(a-c b) \cup O_{e}(a-c b+c) \\
& \cup O_{e}(c-a-2 b a+b) \quad(a+b>b+c), \\
& O_{e}(a b) \otimes O_{e}(c 0)=O_{e}(a+c b) \cup O_{e}(a-c b) \cup O_{e}(a-c b+c) \\
& \cup O_{e}(c-a-2 b a+b) \quad(b+c>a+b>c-b), \\
& O_{e}(a b) \otimes O_{e}(c 0)=O_{e}(a+c b) \cup O_{e}(a-c b) \cup O_{e}(a-c b+c) \\
& \cup O_{e}(c-a-2 b a+b) \quad(a+b<c-b) .
\end{aligned}
$$

The $W_{e}$-orbit $O_{e}\left(r_{\alpha}(a b)\right)$ has the form $O_{e}(-a a+b)$. The products of such orbits with the $W_{e^{-o r b i t}} O_{e}(c 0)$ decompose into $W_{e^{- \text {orbits }} \text { as }}$

$$
\begin{aligned}
& C_{2}: \quad O_{e}(-a a+b) \otimes O_{e}(c 0)=O_{e}(-a-c a+b+c) \cup O_{e}(-a-2 b+c b) \cup O_{e}(c-a a+b) \\
& \cup O_{e}(a+2 b-c b+c) \quad(a+b-c<b), \\
& O_{e}(-a a+b) \otimes O_{e}(c 0)=O_{e}(-a-c a+b+c) \cup O_{e}(c-a-2 b b) \cup O_{e}(c-a a+b) \\
& \cup O_{e}(a+2 b-c c-b) \quad(b>c-a-b, a>c),
\end{aligned}
$$




$$
\begin{aligned}
& O_{e}(-a a+b) \otimes O_{e}(c 0)=O_{e}(-a-c a+b+c) \cup O_{e}(c-a-2 b b) \cup O_{e}(c-a a+b) \\
& \cup O_{e}(a+2 b-c c-b) \quad(b>c-a-b, c>a), \\
& O_{e}(-a a+b) \otimes O_{e}(c 0)=O_{e}(-a-c a+b+c) \cup O_{e}(c-a a+b-c) \cup O_{e}(c-a a+b) \\
& \cup O_{e}(a+2 b-c c-b) \quad(a+b>b+c), \\
& O_{e}(-a a+b) \otimes O_{e}(c 0)=O_{e}(-a-c a+b+c) \cup O_{e}(c-a a+b-c) \cup O_{e}(c-a a+b) \\
& \cup O_{e}(a+2 b-c c-b) \quad(b+c>a+b>c-b), \\
& O_{e}(-a a+b) \otimes O_{e}(c 0)=O_{e}(-a-c a+b+c) \cup O_{e}(c-a a+b-c) \cup O_{e}(c-a a+b) \\
& \cup O_{e}(a+2 b-c c-b) \quad(a+b<c-b) .
\end{aligned}
$$

We also give the following decompositions of $E$-orbits of $C_{2}$ :

$$
\begin{aligned}
& C_{2}: \quad O_{e}(a 0) \otimes O_{e}(b 0)=O_{e}(a+b 0) \cup O_{e}(a-b 0) \cup O_{e}(a-b b) \cup O_{e}(b-a a) \quad(a>b), \\
& O_{e}(a 0) \otimes O_{e}(a 0)=O_{e}(2 a 0) \cup 2 O_{e}(02 a) \cup 4 O_{e}(00) \text {, } \\
& O_{e}(0 a) \otimes O_{e}(0 b)=O_{e}(0 a+b) \cup O_{e}(2 b a-b) \cup O_{e}(-2 b a+b) \cup O_{e}(0 a-b) \quad(a>b) \text {, } \\
& O_{e}(0 a) \otimes O_{e}(0 a)=O_{e}(02 a) \cup 2 O_{e}(2 a \quad 0) \cup 4 O_{e}\left(\begin{array}{lll}
0 & 0
\end{array}\right) \text {, } \\
& O_{e}(a 0) \otimes O_{e}(0 b)=O_{e}(a b) \cup O_{e}(-a a+b) \cup O_{e}(a-2 b b) \cup O_{e}(2 b-a a-b) \quad(a>2 b) \text {, } \\
& O_{e}(a 0) \otimes O_{e}(0 b)=O_{e}(a b) \cup O_{e}(-a a+b) \cup O_{e}(2 b-a a-b) \cup O_{e}(a-2 b b) \quad(2 b>a>b) \text {, } \\
& O_{e}(a 0) \otimes O_{e}(0 b)=O_{e}(a b) \cup O_{e}(-a a+b) \cup O(a b-a) \cup O(-a b) \quad(b>a), \\
& O_{e}(a 0) \otimes O_{e}(0 a)=O_{e}(a a) \cup O_{e}(-a 2 a) \cup 2 O_{e}(a 0) \text {, } \\
& O_{e}(a 0) \otimes O_{e}(02 a)=O_{e}(a 2 a) \cup O_{e}(-a 3 a) \cup O_{e}(a a) \cup O_{e}(-a 2 a), \\
& O_{e}(2 a 0) \otimes O_{e}(0 a)=O_{e}(2 a a) \cup O_{e}(-2 a 3 a) \cup 2 O_{e}(0 a) \text {. }
\end{aligned}
$$

\subsection{Decomposition of products for $G_{2}$}

We give some examples of decomposition of products of $W_{e}$-orbits of $G_{2}$ using $\omega$-coordinates for elements of orbits:

$$
\begin{aligned}
& G_{2}: \quad O_{e}(a 0) \otimes O_{e}(b 0)=O_{e}(a+b 0) \cup O(b-a 3 a) \cup O(2 a+b-3 a) \cup O(2 a-b 3 b-3 a) \\
& \cup O(b-a 3 a-3 b) \cup O_{e}(b-a 0) \quad(a<b<2 a), \\
& O_{e}(a 0) \otimes O_{e}(b 0)=O_{e}(a+b 0) \cup O_{e}(b-a 3 a) \cup O_{e}(2 a+b-3 a) \cup O(b-2 a 3 a) \\
& \cup O_{e}(a+a-3 a) \cup O_{e}(b-a 0) \quad(b>2 a), \\
& O_{e}(a 0) \otimes O_{e}(a 0)=O_{e}(2 a 0) \cup 2 O_{e}(03 a) \cup 2 O_{e}(a 0) \cup 6 O_{e}(00) \text {, } \\
& O_{e}(a 0) \otimes O_{e}(2 a 0)=O_{e}(3 a 0) \cup O_{e}(a 0) \cup O_{e}(a 3 a) \cup O_{e}(4 a-3 a) \cup 2 O_{e}(03 a), \\
& O_{e}(0 a) \otimes O_{e}(0 b)=O_{e}(0 a+b) \cup O_{e}(a b-a) \cup O_{e}(b a-b) \cup O_{e}(b-a 2 a-b) \\
& \cup O_{e}(a b-2 a) \cup O_{e}(0 b-a) \quad(a<b<2 a), \\
& O_{e}(0 a) \otimes O_{e}(0 b)=O_{e}(0 a+b) \cup O_{e}(0 b-a) \cup O_{e}(a b-a) \cup O_{e}(b a-b) \\
& \cup O_{e}(a b-2 a) \cup O_{e}(b-a 2 a-b) \quad(b>2 a), \\
& \left.O_{e}(0 a) \otimes O_{e}(0 a)=O_{e}(02 a) \cup 2 O_{e}(a)\right) \cup 2 O_{e}\left(\begin{array}{ll}
0 & a
\end{array}\right) \cup 6 O_{e}(0 \quad 0), \\
& O_{e}(0 a) \otimes O_{e}(02 a)=O_{e}(03 a) \cup 2 O_{e}(a \quad 0) \cup O_{e}(a a) \cup O_{e}(2 a-a) \cup O_{e}(0 a) .
\end{aligned}
$$

\section{Decomposition of $W_{e}$-orbit functions into $W_{e}^{\prime}$-orbit functions}

For these decompositions it is enough to obtain the corresponding decompositions for signed $W$-orbits and then to make a corresponding separation of $W_{e}$-orbits. For this reason, we shall deal mainly with signed orbits. Our reasoning here is similar to that of Section 4 in [7]. 


\subsection{Introduction}

Let $R$ be a root system with a Weyl group $W$ and let $R^{\prime}$ be another root system which is a subset of the set $R$. Then the Weyl group $W^{\prime}$ for $R^{\prime}$ can be considered as a subgroup of $W$. Moreover, $W_{e}^{\prime}$ is a subgroup of the even Weyl group $W_{e}$.

Let $O_{e}^{W}(\lambda)$ be a $W_{e}$-orbit. The set of points of $O_{e}^{W}(\lambda)$ is invariant with respect to $W_{e}^{\prime}$. This means that the orbit $O_{e}^{W}(\lambda)$ consists of $W_{e}^{\prime}$-orbits. In this section we deal with representing $O_{e}^{W}(\lambda)$ as a union of $W_{e}^{\prime}$-orbits. Properties of such a representation depend on root systems $R$ and $R^{\prime}$ (or on Weyl groups $W$ and $W^{\prime}$ ). We distinguish two cases:

Case 1. Root systems $R$ and $R^{\prime}$ span vector spaces of the same dimension. In this case Weyl chambers for $W$ are smaller than Weyl chambers for $W^{\prime}$. Moreover, each Weyl chamber for $W^{\prime}$ consists of $\left|W / W^{\prime}\right|$ chambers for $W$. Therefore, an even Weyl chamber for $W_{e}^{\prime}$ consists of $\left|W / W^{\prime}\right|=\left|W_{e} / W_{e}^{\prime}\right|$ even Weyl chambers of $W_{e}$. Let $D_{e}^{+}$be an even dominant Weyl chamber for the root system $R$. Then the even dominant Weyl chamber for $W_{e}^{\prime}$ consists of $W_{e}$-chambers $w_{i} D_{e}^{+}, i=1,2, \ldots, k, k=\left|W / W^{\prime}\right|$, where $w_{i}, i=1,2, \ldots, k$, are representatives of cosets in $W_{e} / W_{e}^{\prime}$. If $\lambda$ does not lie on any wall of the even dominant Weyl chamber $D_{e}^{+}$, then

$$
O_{e}^{W}(\lambda)=\bigcup_{i=1}^{k} O_{e}^{W^{\prime}}\left(w_{i} \lambda\right),
$$

where $O_{e}^{W^{\prime}}$ are $W_{e}^{\prime}$-orbits.

Representing $\lambda$ by coordinates in $\omega$-basis it is necessary to take into account that coordinates of the same point in $\omega$-bases related to the root systems $R$ and $R^{\prime}$ are different. There exist matrices connecting coordinates in these different $\omega$-bases (see [29]).

To the decomposition (7.1) there corresponds the following expansion for $E$-orbit functions:

$$
E_{\lambda}^{(W)}(x)=\sum_{i=1}^{k} E_{w_{i} \lambda}^{\left(W^{\prime}\right)}(x) .
$$

Case 2. Root systems $R$ and $R^{\prime}$ span vector spaces of different dimensions. This case is more complicated. In order to represent $O_{e}^{W}(\lambda)$ as a union of $W_{e}^{\prime}$-orbits, it is necessary to project points $\mu$ of $O_{e}^{W}(\lambda)$ to the vector subspace $E_{n^{\prime}}$ spanned by $R^{\prime}$ and to select in the set of these projected points dominant points with respect to the root system $R^{\prime}$. Note that under projection, different points of $O_{e}^{W}(\lambda)$ can give the same point in $E_{n^{\prime}}$. This leads to appearing of coinciding $W_{e}^{\prime}$-orbits in a representation of $O_{e}^{W}(\lambda)$ as a union of $W_{e}^{\prime}$-orbits.

Under expansion of an $E^{W}$-orbit function $E_{\lambda}^{(W)}(x)$ into $E^{\left(W^{\prime}\right)}$-orbit functions we have to

consider $E_{\lambda}^{(W)}(x)$ on the subspace $E_{n^{\prime}} \subset E_{n}$ and to take into account the corresponding decomposition of the orbit $O_{e}^{W}(\lambda)$. For this reason, below in this section we consider decomposition of $W_{e}$-orbits into $W_{e}^{\prime}$-orbits. They uniquely determine the corresponding expansions for $E$-orbit functions.

\subsection{Decomposition of $W_{e}\left(A_{n}\right)$-orbits into $W_{e}\left(A_{n-1}\right)$-orbits}

Below we shall consider decompositions for $W_{e}\left(A_{n}\right)$-orbits $O_{e}(\lambda)$ and $O_{e}\left(r_{\alpha} \lambda\right)$ such that $\lambda$ is strictly dominant.

If $\lambda$ is not strictly dominant, then $W_{e}\left(A_{n}\right)$-orbit $O_{e}(\lambda) \equiv O(\lambda)$ coincides with the $W\left(A_{n}\right)$ orbit $O(\lambda)$. In this case, in order to decompose $W_{e}\left(A_{n}\right)$-orbit $O_{e}(\lambda)$ into $W_{e}\left(A_{n-1}\right)$-orbits we have to decompose the $W\left(A_{n}\right)$-orbit $O(\lambda)$ into $W\left(A_{n-1}\right)$-orbits and then to split $W\left(A_{n-1}\right)$-orbits into $W_{e}\left(A_{n-1}\right)$-orbits. Namely, if a $W\left(A_{n-1}\right)$-orbit $O(\mu)$ is such that $\mu$ is not strictly dominant, then 
$O(\mu)$ is in fact a $W_{e}\left(A_{n-1}\right)$-orbit. If $\mu$ is strictly dominant, then $O(\mu)$ consists of two $W_{e}\left(A_{n-1}\right)$ orbits $O_{e}(\mu)$ and $O\left(r_{\alpha} \mu\right)$, where $\alpha$ is a root of $A_{n-1}$. Thus, if $\lambda$ is not strictly dominant, then decomposition of $W_{e}\left(A_{n}\right)$-orbits into $W_{e}\left(A_{n-1}\right)$-orbits are reduced to decomposition of $W\left(A_{n}\right)$ orbits into $W\left(A_{n-1}\right)$-orbits. The last decomposition are studied in Subsection 4.5 in [7].

So, let $\lambda$ be a strictly dominant element for $A_{n}$. It is convenient to fulfil the decomposition of $W_{e}\left(A_{n}\right)$-orbits $O_{e}(\lambda)$ and $O_{e}\left(r_{\alpha} \lambda\right)$ by using a corresponding decomposition of a signed $W\left(A_{n}\right)$ orbit $O^{ \pm}(\lambda)$ into signed $W\left(A_{n-1}\right)$-orbits considered in Subsection 8.2 in [8]. For this we have to take into account that a signed $W\left(A_{n}\right)$-orbit $O^{ \pm}(\lambda)$ consists of two $W_{e}\left(A_{n}\right)$-orbits. One of them consists of points with the sign + and the second with the sign - .

For such decomposition it is convenient to represent orbit elements in orthogonal coordinates (see Section 3). Let $m_{1}, m_{2}, \ldots, m_{n+1}$ be orthogonal coordinates of a strictly dominant element $\lambda$, that is,

$$
m_{1}>m_{2}>\cdots>m_{n}>m_{n+1} .
$$

The orthogonal coordinates $m_{1}, m_{2}, \ldots, m_{n+1}$ satisfy the conditions $m_{1}+m_{2}+\cdots+m_{n+1}=0$. However, we may add to all coordinates $m_{i}$ the same real number, since under this procedure the $\omega$-coordinates $\lambda_{i}=m_{i}-m_{i+1}, i=1,2, \ldots, n$ do not change (see Section 3 ).

Let $O^{ \pm}(\lambda) \equiv O^{ \pm}\left(m_{1}, m_{2}, \ldots, m_{n+1}\right)$ be a signed $W\left(A_{n}\right)$-orbit with dominant element $\lambda=$ $\left(m_{1}, m_{2}, \ldots, m_{n+1}\right)$. This orbit consists of all points

$$
w\left(m_{1}, m_{2}, \ldots, m_{n+1}\right)=\left(m_{i_{1}}, m_{i_{2}}, \ldots, m_{i_{n+1}}\right), \quad w \in W\left(A_{n}\right),
$$

where $\left(i_{1}, i_{2}, \ldots, i_{n+1}\right)$ is a permutation of the numbers $1,2, \ldots, n+1$, determined by $w$. The sign of $(\operatorname{det} w)$ is attached to such a point. Points of $O^{ \pm}(\lambda)$ belong to the Euclidean space $E_{n+1}$. We restrict these points to the subspace $E_{n}$, spanned by the simple roots $\alpha_{1}, \alpha_{2}, \ldots, \alpha_{n-1}$ of $A_{n}$, which form a set of simple roots of $A_{n-1}$. This restriction is reduced to removing the last coordinate $m_{i_{n+1}}$ in points $\left(m_{i_{1}}, m_{i_{2}}, \ldots, m_{i_{n+1}}\right)$ of the signed orbit $O^{ \pm}(\lambda)$ (see $\left.(7.2)\right)$. As a result, we obtain the set of points

$$
\left(m_{i_{1}}, m_{i_{2}}, \ldots, m_{i_{n}}\right)
$$

received from the points (7.2). The point (7.3) is dominant if and only if

$$
m_{i_{1}} \geq m_{i_{2}} \geq \cdots \geq m_{i_{n}} .
$$

It is easy to see that after restriction to $E_{n}$ (that is, under removing the last coordinate) we obtain from the set of points (7.2) the following set of dominant elements:

$$
\left(m_{1}, \ldots, m_{i-1}, \hat{m}_{i}, m_{i+1}, \ldots, m_{n+1}\right), \quad i=1,2, \ldots, n+1,
$$

where a hat over $m_{i}$ means that the coordinate $m_{i}$ must be omitted.

Thus, the signed $W\left(A_{n}\right)$-orbit $O^{ \pm}\left(m_{1}, m_{2}, \ldots, m_{n+1}\right)$ consists of the following signed $W\left(A_{n-1}\right)$-orbits:

$$
O^{ \pm}\left(m_{1}, \ldots, m_{i-1}, \hat{m}_{i}, m_{i+1}, \ldots, m_{n+1}\right), \quad i=1,2, \ldots, n+1 .
$$

Each of these signed orbits must be taken with a coefficient +1 or -1 . Moreover, a coefficient at the orbit $O^{ \pm}\left(m_{1}, \ldots, m_{i-1}, \hat{m}_{i}, m_{i+1}, \ldots, m_{n+1}\right)$ is 1 , if after $\hat{m}_{i}$ in the point

$$
\left(m_{1}, \ldots, m_{i-1}, \hat{m}_{i}, m_{i+1}, \ldots, m_{n+1}\right)
$$

there exists an even number of coordinates, and -1 otherwise (see Section 8.2 in [8]). These statements can be written in the form

$$
O_{W}^{ \pm}\left(m_{1}, m_{2}, \ldots, m_{n+1}\right)=\bigcup_{i=1}^{n+1}\left(\operatorname{det} w\left(m_{i}\right)\right) O_{W^{\prime}}^{ \pm}\left(m_{1}, \ldots, m_{i-1}, \hat{m}_{i}, m_{i+1}, \ldots, m_{n+1}\right),
$$


where $w\left(m_{i}\right)$ is the permutation which send the coordinate $m_{i}$ to the end, not changing an order of other coordinates.

Now we have to split the left and the right hand sides of (7.4) into two parts: the first part has to consist of points with the sign plus and the second with the sign minus. This splitting for the left hand side leads to two $W_{e}\left(A_{n}\right)$-orbits $O_{e}(\lambda)$ and $O_{e}\left(r_{\alpha} \lambda\right)$. Each signed $W\left(A_{n-1}\right)$-orbit on the right hand side splits into two $W\left(A_{n-1}\right)$-orbits, one contains points with the sign plus and other with the sign minus; one of them is contained in $O_{e}(\lambda)$ and another in $O_{e}\left(r_{\alpha} \lambda\right)$. If $\operatorname{det} w\left(m_{i}\right)=1$, then the $W_{e}\left(A_{n-1}\right)$-orbit of $O_{W^{\prime}}^{ \pm}\left(m_{1}, \ldots, m_{i-1}, \hat{m}_{i}, m_{i+1}, \ldots, m_{n+1}\right)$ with points having the sign plus belongs to $O_{e}(\lambda)$. The $W_{e}\left(A_{n-1}\right)$-orbit with points having the sign minus belongs to $O_{e}\left(r_{\alpha} \lambda\right)$. If $\operatorname{det} w\left(m_{i}\right)=-1$, then the $W_{e}\left(A_{n-1}\right)$-orbit of $O_{W^{\prime}}^{ \pm}\left(m_{1}, \ldots, m_{i-1}, \hat{m}_{i}, m_{i+1}\right.$, $\left.\ldots, m_{n+1}\right)$ with points having the sign plus belongs to $O_{e}\left(r_{\alpha} \lambda\right)$ and the $W_{e}\left(A_{n-1}\right)$-orbit with points having the sign minus belongs to $O_{e}(\lambda)$. Fulfilling this splitting we obtain lists of $W_{e}\left(A_{n-1}\right)$-orbits, which are contained in $W_{e}\left(A_{n}\right)$-orbits $O_{e}(\lambda)$ and $O_{e}\left(r_{\alpha} \lambda\right)$.

\subsection{Decomposition of $W_{e}\left(B_{n}\right)$-orbits into $W_{e}\left(B_{n-1}\right)$-orbits and of $W_{e}\left(C_{n}\right)$-orbits into $W_{e}\left(C_{n-1}\right)$-orbits}

Decomposition of $W_{e}\left(B_{n}\right)$-orbits and decomposition of $W_{e}\left(C_{n}\right)$-orbits are fulfilled in the same way. For this reason, we give a proof only for the case of $W_{e}\left(C_{n}\right)$-orbits. As in the previous case for fulfilling the decompositions we use signed $W$-orbits.

A set of simple roots of $C_{n}$ consists of roots $\alpha_{1}, \alpha_{2}, \ldots, \alpha_{n}$. The roots $\alpha_{2}, \ldots, \alpha_{n}$ constitute a set of simple roots of $C_{n-1}$. They span the subspace $E_{n-1}$.

We determine elements $\lambda$ of $E_{n}$ by using orthogonal coordinates $m_{1}, m_{2}, \ldots, m_{n}$. Then $\lambda$ is strictly dominant if and only if

$$
m_{1}>m_{2}>\cdots>m_{n}>0 .
$$

Then the signed $W\left(C_{n}\right)$-orbit $O^{ \pm}(\lambda)$ consists of all points

$$
w\left(m_{1}, m_{2}, \ldots, m_{n}\right)=\left( \pm m_{i_{1}}, \pm m_{i_{2}}, \ldots, \pm m_{i_{n}}\right), \quad w \in W\left(C_{n}\right)
$$

where $\left(i_{1}, i_{2}, \ldots, i_{n}\right)$ is a permutation of the set $1,2, \ldots, n$, and all combinations of signs are possible.

Restriction of elements (7.5) to the vector subspace $E_{n-1}$, defined above, reduces to removing the first coordinate $\pm m_{i_{1}}$ in (7.5). As a result, we obtain from the set of points (7.5) the collection

$$
\left( \pm m_{i_{2}}, \pm m_{i_{3}}, \ldots, \pm m_{i_{n}}\right), \quad w \in W\left(C_{n}\right) .
$$

Only the points $\left(m_{i_{2}}, m_{i_{3}}, \ldots, m_{i_{n-1}}, m_{i_{n}}\right)$ with positive coordinates may be dominant. Moreover, such a point is dominant if and only if

$$
m_{i_{2}}>m_{i_{3}}>\cdots>m_{i_{n}}
$$

Therefore, under restriction of points (7.5) to $E_{n-1}$ we obtain the following strictly $W\left(C_{n-1}\right)$ dominant elements:

$$
\left(m_{1}, m_{2}, \ldots, m_{i-1}, \hat{m}_{i}, m_{i+1}, \ldots, m_{n}\right), \quad i=1,2, \ldots, n
$$

where a hat over $m_{i}$ means that the coordinate $m_{i}$ must be omitted. Moreover, the element (7.6) with fixed $i$ can be obtained from two elements in (7.5), namely, from $\left(m_{1}, m_{2}, \ldots, m_{i-1}, \pm m_{i}\right.$, $\left.m_{i+1}, \ldots, m_{n}\right)$. In the signed orbit $O^{ \pm}\left(m_{1}, m_{2}, \ldots, m_{n}\right)$ these two elements have opposite signs. 
Thus, the signed $W\left(C_{n}\right)$-orbits $O^{ \pm}\left(m_{1}, m_{2}, \ldots, m_{n}\right)$ consists of the following signed $W\left(C_{n-1}\right)$ orbits:

$$
\begin{aligned}
& O^{ \pm}\left(m_{1}, m_{2}, \ldots, m_{i-1}, \hat{m}_{i}, m_{i+1}, \ldots, m_{n}\right) \\
& \quad \equiv O^{ \pm}\left(m_{1}, \ldots, \hat{m}_{i}, \ldots, m_{n}\right), \quad i=1,2, \ldots, n .
\end{aligned}
$$

Each such signed $W\left(C_{n-1}\right)$-orbit is contained in $O^{ \pm}\left(m_{1}, m_{2}, \ldots, m_{n}\right)$ twice with opposite signs. Since the signed $W\left(C_{n}\right)$-orbit $O^{ \pm}\left(m_{1}, m_{2}, \ldots, m_{n}\right)$ consists of two $W_{e}\left(C_{n}\right)$-orbits (one consists of points with the sign + and the second with the sign -$)$, then it follows from these assertions that the $W_{e}\left(C_{n}\right)$-orbits $O_{e}\left(m_{1}, m_{2}, \ldots, m_{n}\right)$ and $O_{e}\left(r_{\alpha}\left(m_{1}, m_{2}, \ldots, m_{n}\right)\right)$ consist of the same set of $W_{e}\left(C_{n-1}\right)$-orbits, namely, of $W_{e}\left(C_{n-1}\right)$-orbits which are contained in the signed $W\left(C_{n-1}\right)$-orbits $(7.7)$. This set consists of the $W_{e}\left(C_{n-1}\right)$-orbits $O^{ \pm}\left(m_{1}, \ldots, \hat{m}_{i}, \ldots, m_{n}\right)$ and $O^{ \pm}\left(r_{\beta}\left(m_{1}, \ldots, \hat{m}_{i}, \ldots, m_{n}\right)\right), i=1,2, \ldots, n$, where $\beta$ is a root of $C_{n-1}$.

For $W_{e}\left(B_{n}\right)$-orbits we have similar assertions. A $W_{e}\left(B_{n}\right)$-orbit $O_{e}\left(m_{1}, m_{2}, \ldots, m_{n}\right), m_{1}>$ $m_{2}>\cdots>m_{n}>0$, consists of $W_{e}\left(B_{n-1}\right)$-orbits which are contained in the signed $W^{ \pm}\left(B_{n}\right)$ orbit

$$
O^{ \pm}\left(m_{1}, m_{2}, \ldots, m_{i-1}, \hat{m}_{i}, m_{i+1}, \ldots, m_{n}\right), \quad i=1,2, \ldots, n,
$$

and each such $W_{e}\left(B_{n-1}\right)$-orbit is contained in the decomposition only once.

\subsection{Decomposition of $W_{e}\left(D_{n}\right)$-orbits into $W_{e}\left(D_{n-1}\right)$-orbits}

Assume that $\alpha_{1}, \alpha_{2}, \ldots, \alpha_{n}$ is the set of simple roots of $D_{n}, n>4$. Then $\alpha_{2}, \ldots, \alpha_{n}$ are simple roots of $D_{n-1}$. The last roots span the subspace $E_{n-1}$.

For elements $\lambda$ of $E_{n}$ we use orthogonal coordinates $m_{1}, m_{2}, \ldots, m_{n}$. Then $\lambda$ is strictly dominant if and only if $m_{1}>m_{2}>\cdots>m_{n-1}>\left|m_{n}\right|$. We assume that $\lambda$ satisfies the condition

$$
m_{1}>m_{2}>\cdots>m_{n}>0 \text {. }
$$

Then the signed $W\left(D_{n}\right)$-orbit $O^{ \pm}(\lambda)$ consists of all points

$$
w\left(m_{1}, m_{2}, \ldots, m_{n}\right)=\left( \pm m_{i_{1}}, \pm m_{i_{2}}, \ldots, \pm m_{i_{n}}\right), \quad w \in W_{D_{n}},
$$

where $\left(i_{1}, i_{2}, \ldots, i_{n}\right)$ is a permutation of the numbers $1,2, \ldots, n$ and there exists an even number of signs -. Restriction of elements (7.8) to the subspace $E_{n-1}$ reduces to removing the first coordinate $\pm m_{i_{1}}$ in (7.8). As a result, we obtain from the set of points (7.8) the collection

$$
\left( \pm m_{i_{2}}, \pm m_{i_{3}}, \ldots, \pm m_{i_{n}}\right), \quad w \in W\left(D_{n}\right)
$$

where a number of signs - may be either even or odd. Only points of the form $\left(m_{i_{2}}, m_{i_{3}}, \ldots\right.$, $\left.m_{i_{n-1}}, \pm m_{i_{n}}\right)$ may be dominant. Moreover, such a point is dominant if and only if

$$
m_{i_{2}}>m_{i_{3}}>\cdots>m_{i_{n-1}}>\left|m_{i_{n}}\right| .
$$

Therefore, under restriction of points (7.8) to $E_{n-1}$ we obtain the following $W\left(D_{n-1}\right)$-dominant elements:

$$
\left(m_{1}, m_{2}, \ldots, m_{i-1}, \hat{m}_{i}, m_{i+1}, \ldots, m_{n-1}, \pm m_{n}\right), \quad i=1,2, \ldots, n,
$$

where a hat over $m_{i}$ means that the coordinate $m_{i}$ must be omitted. Moreover, the element (7.9) with fixed $i$ can be obtained only from one element in (7.8), namely, from element $\left(m_{1}, m_{2}, \ldots, m_{i-1}, \pm m_{i}, m_{i+1}, \ldots, \pm m_{n}\right)$, where at $m_{i}$ and $m_{n}$ signs are coinciding. 
Thus, the signed $W\left(D_{n}\right)$-orbit $O^{ \pm}\left(m_{1}, m_{2}, \ldots, m_{n}\right)$ with $m_{1}>m_{2}>\cdots>m_{n}>0$ consists of the following signed $W\left(D_{n-1}\right)$-orbits:

$$
O^{ \pm}\left(m_{1}, m_{2}, \ldots, m_{i-1}, \hat{m}_{i}, m_{i+1}, \ldots, \pm m_{n}\right), \quad i=1,2, \ldots, n .
$$

Each such signed $W\left(D_{n-1}\right)$-orbit is contained in $O^{ \pm}\left(m_{1}, m_{2}, \ldots, m_{n}\right)$ only once (with sign + or sign -). A sign of such an orbit depends on a number $i$ and does not depend on a sign at $m_{n}$. This sign is + if after $\hat{m}_{i}$ in (7.10) there exists on even number of coordinates and -1 otherwise.

Now we split the signed $W\left(D_{n}\right)$-orbit $O^{ \pm}\left(m_{1}, m_{2}, \ldots, m_{n}\right)$ into two $W_{e}\left(D_{n}\right)$-orbits $O_{e}\left(m_{1}\right.$, $\left.m_{2}, \ldots, m_{n}\right)$ and $O_{e}\left(r_{\alpha}\left(m_{1}, m_{2}, \ldots, m_{n}\right)\right)$. Next, we split each of the signed $W\left(D_{n-1}\right)$-orbits (7.10) also into two $W_{e}\left(D_{n-1}\right)$-orbits

$$
\begin{array}{lr}
O_{e}\left(m_{1}, m_{2}, \ldots, m_{i-1}, \hat{m}_{i}, m_{i+1}, \ldots, \pm m_{n}\right), \quad i=1,2, \ldots, n . \\
O^{e}\left(r_{\beta}\left(m_{1}, m_{2}, \ldots, m_{i-1}, \hat{m}_{i}, m_{i+1}, \ldots, \pm m_{n}\right)\right), \quad i=1,2, \ldots, n .
\end{array}
$$

It is necessary to split these $W_{e}\left(D_{n-1}\right)$-orbits into two parts which constitute the $W_{e}\left(D_{n}\right)$ orbits $O_{e}\left(m_{1}, m_{2}, \ldots, m_{n}\right)$ and $O_{e}\left(r_{\alpha}\left(m_{1}, m_{2}, \ldots, m_{n}\right)\right)$. This is done as follows. If a fixed signed orbit from (7.10) is contained in the signed $W\left(D_{n}\right)$-orbit $O^{ \pm}\left(m_{1}, m_{2}, \ldots, m_{n}\right)$ with the sign + , then the corresponding $W_{e}\left(D_{n-1}\right)$-orbit (7.11) is contained in the $W_{e}\left(D_{n}\right)$-orbit $O_{e}\left(m_{1}, m_{2}, \ldots, m_{n}\right)$ and the $W_{e}\left(D_{n-1}\right)$-orbit $(7.12)$ is contained in $O_{e}\left(r_{\alpha}\left(m_{1}, m_{2}, \ldots, m_{n}\right)\right)$. If a fixed signed $W\left(D_{n-1}\right)$-orbit (7.10) is contained in $O^{ \pm}\left(m_{1}, m_{2}, \ldots, m_{n}\right)$ with the sign -, then the corresponding $W_{e}\left(D_{n-1}\right)$-orbit $(7.11)$ is contained in the $W_{e}\left(D_{n}\right)$-orbit $O_{e}\left(r_{\alpha}\left(m_{1}, m_{2}\right.\right.$, $\left.\left.\ldots, m_{n}\right)\right)$ and the $W_{e}\left(D_{n-1}\right)$-orbit $(7.12)$ is contained in $O_{e}\left(m_{1}, m_{2}, \ldots, m_{n}\right)$. Therefore, the $W_{e}\left(D_{n}\right)$-orbit $O_{e}\left(m_{1}, m_{2}, \ldots, m_{n}\right)$ consists of $W_{e}\left(D_{n-1}\right)$-orbit (7.11) such that $n-i$ is an even integer and of $W_{e}\left(D_{n-1}\right)$-orbit (7.12) such that $n-i$ is odd. The $W_{e}\left(D_{n}\right)$-orbit $O_{e}\left(r_{\alpha}\left(m_{1}, m_{2}\right.\right.$, $\left.\left.\ldots, m_{n}\right)\right)$ consists of $W_{e}\left(D_{n-1}\right)$-orbit (7.11) such that $n-i$ is odd and of $W_{e}\left(D_{n-1}\right)$-orbit (7.12) such that $n-i$ is even.

It is shown similarly that the $W_{e}\left(D_{n}\right)$-orbits

$$
O_{e}\left(m_{1}, \ldots, m_{n-1},-m_{n}\right), \quad O_{e}\left(r_{\alpha}\left(m_{1}, \ldots, m_{n-1},-m_{n}\right)\right),
$$

with $m_{1}>m_{2}>\cdots>m_{n}>0$ consists of the same $W_{e}\left(D_{n-1}\right)$-orbits as the $W_{e}\left(D_{n}\right)$-orbits $O_{e}\left(m_{1}, \ldots, m_{n-1}, m_{n}\right)$ and $O_{e}\left(r_{\alpha}\left(m_{1}, \ldots, m_{n-1}, m_{n}\right)\right)$ with the same numbers $m_{1}, \ldots, m_{n-1}, m_{n}$ do, respectively.

\section{$8 \quad E$-orbit function transforms}

As in the case of symmetric and antisymmetric orbit functions, $E$-orbit functions determine certain orbit function transforms which generalize the Fourier transform (in the case of symmetric orbit functions these transforms generalize the cosine transform and in the case of antisymmetric orbit functions these transforms generalize the sine transform) $[7,8,10]$.

As in the case of symmetric and antisymmetric orbit functions, $E$-orbit functions determine three types of orbit function transforms: the first one is related to the $E$-orbit functions $E_{\lambda}(x)$ with integral $\lambda$, the second one is related to $E_{\lambda}(x)$ with real values of coordinates of $\lambda$, and the third one is the related discrete transform.

\subsection{Expansion in $\boldsymbol{E}$-orbit functions on $\boldsymbol{F}_{e}$}

The aim of this subsection is to obtain formulas for expansions of functions on the closure of the fundamental domain $F_{e}$ of the even affine Weyl group $W_{e}^{\text {aff }}$ in $E$-orbit functions $E_{\lambda}(x)$ with integral $\lambda$. 
Let us start with the usual Fourier expansion of functions on $E_{n}$,

$$
f(x)=\sum_{\lambda \in \mathbb{Z}^{n}} c_{\lambda} e^{2 \pi\langle\lambda, x\rangle} .
$$

with coefficients

$$
c_{\lambda}=\int_{x \in \mathbf{T}} f(x) e^{-2 \pi\langle\lambda, x\rangle} d x
$$

where $\mathrm{T}$ is a torus in $E_{n}$.

Let the function $f(x)$ be invariant with respect to the even Weyl group $W_{e}$. It is easy to check that the coefficients $c_{\lambda}$ are also $W_{e}$-invariant, $c_{w \lambda}=c_{\lambda}, w \in W_{e}$. Replace in (8.2) $\lambda$ by $w \lambda$, $w \in W_{e}$, and sum up both side of (8.2) over $w \in W_{e}$. Then instead of (8.2) we obtain

$$
c_{\lambda}=\left|W_{e}\right|^{-1} \int_{\mathrm{T}} f(x) \hat{E}_{\lambda}(x) d x
$$

where $\hat{E}_{\lambda}(x)=\sum_{w \in W_{e}} e^{2 \pi \mathrm{i}\langle w \lambda, x\rangle}$. (We have taken into account that both $f(x)$ and $E_{\lambda}(x)$ are $W_{e}$-invariant.) This formula can be written as

$$
c_{\lambda}=\int_{\overline{F_{e}}} f(x) \hat{E}_{\lambda}(x) d x=\left|W_{\lambda}\right| \int_{\overline{F_{e}}} f(x) E_{\lambda}(x) d x,
$$

where $W_{\lambda}$ is the subgroup of $W_{e}$ consisting of elements leaving $\lambda$ invariant.

Similarly, starting from (8.1), we obtain an inverse formula:

$$
f(x)=\sum_{\lambda \in P_{e}^{+}} c_{\lambda} \overline{E_{\lambda}(x)}
$$

where $P_{e}^{+}$is the set of integral elements from $D_{e}^{+}$. For the transforms (8.3) and (8.4) the Plancherel formula

$$
\int_{\overline{F_{e}}}|f(x)|^{2} d x=\sum_{\lambda \in P_{e}^{+}}\left|W_{\lambda}\right|^{-1}\left|c_{\lambda}\right|^{2}
$$

holds, which means that the Hilbert spaces with the appropriate scalar products are isometric.

Formula (8.4) is the symmetrized (by means of the group $W_{e}$ ) Fourier transform of the function $f(x)$. Formula (8.3) gives an inverse transform. These formulas give the E-orbit function transforms corresponding to $E$-orbit functions $E_{\lambda}, \lambda \in P_{e}^{+}$.

Let $\mathcal{L}^{2}\left(F_{e}\right)$ denote the Hilbert space of functions on the closure of the fundamental domain $F_{e}$ of the group $W_{e}^{\text {aff }}$ with the scalar product

$$
\left\langle f_{1}, f_{2}\right\rangle=\int_{\overline{F_{e}}} f_{1}(x) \overline{f_{2}(x)} d x .
$$

The formulas (8.3) and (8.4) show that the set of E-orbit functions $E_{\lambda}, \lambda \in P_{e}^{+}$, form an orthogonal basis of $\mathcal{L}^{2}\left(F_{e}\right)$.

\section{2 $E$-orbit function transform on even dominant Weyl chamber}

The expansion (8.4) of functions on the domain $\overline{F_{e}}$ is an expansion in the $E$-orbit functions $E_{\lambda}(x)$ with integral elements $\lambda$. The $E$-orbit functions $E_{\lambda}(x)$ with $\lambda$ lying in the even dominant Weyl chamber (and not obligatory integral) are not invariant with respect to the corresponding even 
affine Weyl group $W_{e}^{\text {aff }}$. They are invariant only with respect to the even Weyl group $W_{e}$. A fundamental domain of $W_{e}$ coincides with the even dominant Weyl chamber $D_{e}^{+}=D_{+} \cup r_{\alpha} D_{+}$. For this reason, the $E$-orbit functions $E_{\lambda}(x), \lambda \in D_{e}^{+}$, determine another orbit function transform (a transform on $D_{e}^{+}$).

We began with the usual Fourier transforms on $\mathbb{R}^{n}$ :

$$
\begin{aligned}
& \tilde{f}(\lambda)=\int_{\mathbb{R}^{n}} f(x) e^{2 \pi \mathrm{i}\langle\lambda, x\rangle} d x, \\
& f(x)=\int_{\mathbb{R}^{n}} \tilde{f}(\lambda) e^{-2 \pi \mathrm{i}\langle\lambda, x\rangle} d \lambda .
\end{aligned}
$$

Let the function $f(x)$ be invariant with respect to the even Weyl group $W_{e}$, that is, $f(w x)=$ $(\operatorname{det} w) f(x), w \in W_{e}$. The function $\tilde{f}(\lambda)$ is also invariant with respect to the even Weyl group $W_{e}$. Replace in (8.5) $\lambda$ by $w \lambda, w \in W_{e}$, and sum up these both side over $w \in W_{e}$. Then instead of (8.5) we obtain

$$
\tilde{f}(\lambda)=\left|W_{e}\right|^{-1} \int_{\mathbb{R}^{n}} f(x) \hat{E}_{\lambda}(x) d x, \quad \lambda \in D_{e}^{+},
$$

Therefore,

$$
\tilde{f}(\lambda)=\int_{D_{e}^{+}} f(x) \hat{E}_{\lambda}(x) d x, \quad \lambda \in D_{e}^{+},
$$

where we have taken into account that $f(x)$ is invariant with respect to $W_{e}$.

Similarly, starting from (8.6), we obtain the inverse formula:

$$
f(x)=\int_{D_{e}^{+}} \tilde{f}(\lambda) \overline{\hat{E}_{\lambda}(x)} d \lambda .
$$

For the transforms (8.7) and (8.8) the Plancherel formula

$$
\int_{D_{e}^{+}}|f(x)|^{2} d x=\int_{D_{e}^{+}}|\tilde{f}(\lambda)|^{2} d \lambda
$$

holds.

\section{Finite $E$-orbit function transforms}

\subsection{Introduction}

It is possible to introduce finite $E$-orbit function transforms, based on $E$-orbit functions (see [10]). It is done in the same way as in the case of symmetric orbit functions in [7] by using the results of paper [11]. Finite $E$-orbit function transforms are generalizations of the finite (discrete) Fourier transforms, which are defined as follows.

Let us fix a positive integer $N$ and consider the numbers

$$
e_{m n}:=N^{-1 / 2} \exp (2 \pi \mathrm{i} m n / N), \quad m, n=1,2, \ldots, N .
$$

The matrix $\left(e_{m n}\right)_{m, n=1}^{N}$ is unitary, that is,

$$
\sum_{k} e_{m k} \overline{e_{n k}}=\delta_{m n}, \quad \sum_{k} e_{k m} \overline{e_{k n}}=\delta_{m n}
$$


Let $f(n)$ be a function of $n \in\{1,2, \ldots, N\}$. We may consider the transform

$$
\sum_{n=1}^{N} f(n) e_{m n} \equiv N^{-1 / 2} \sum_{n=1}^{N} f(n) \exp (2 \pi \mathrm{i} m n / N)=\tilde{f}(m)
$$

Then due to unitarity of the matrix $\left(e_{m n}\right)_{m, n=1}^{N}$, we express $f(n)$ as a linear combination of conjugates of the functions (9.1):

$$
f(n)=N^{-1 / 2} \sum_{m=1}^{N} \tilde{f}(m) \exp (-2 \pi \mathrm{i} m n / N) .
$$

The function $\tilde{f}(m)$ is a finite Fourier transform of $f(n)$. This transform is a linear map. The formula (9.4) gives an inverse transform. The Plancherel formula

$$
\sum_{m=1}^{N}|\tilde{f}(m)|^{2}=\sum_{n=1}^{N}|f(n)|^{2}
$$

holds for transforms (9.3) and (9.4). This means that the finite Fourier transform conserves the norm introduced on the space of functions on $\{1,2, \ldots, N\}$.

The finite Fourier transform on the $r$-dimensional linear space $E_{r}$ is defined similarly. We again fix a positive integer $N$. Let $\mathbf{m}=\left(m_{1}, m_{2}, \ldots, m_{r}\right)$ be an $r$-tuple of integers such that each $m_{i}$ runs over the integers $1,2, \ldots, N$. Then the finite Fourier transform on $E_{r}$ is given by the kernel

$$
e_{\mathbf{m n}}:=e_{m_{1} n_{1}} e_{m_{2} n_{2}} \cdots e_{m_{r} n_{r}}=N^{-r / 2} \exp (2 \pi \mathbf{i m} \cdot \mathbf{n} / N),
$$

where $\mathbf{m} \cdot \mathbf{n}=m_{1} n_{1}+m_{2} n_{2}+\cdots+m_{r} n_{r}$. If $F(\mathbf{m})$ is a function of $r$-tuples $\mathbf{m}, m_{i} \in\{1,2, \ldots, N\}$, then the finite Fourier transform of $F$ is given by

$$
\tilde{F}(\mathbf{n})=N^{-r / 2} \sum_{\mathbf{m}} F(\mathbf{m}) \exp (2 \pi \mathrm{im} \cdot \mathbf{n} / N) .
$$

The inverse transform is

$$
F(\mathbf{m})=N^{-r / 2} \sum_{\mathbf{n}} \tilde{F}(\mathbf{n}) \exp (-2 \pi \mathrm{im} \cdot \mathbf{n} / N)
$$

The corresponding Plancherel formula is of the form $\sum_{\mathbf{m}}|F(\mathbf{m})|^{2}=\sum_{\mathbf{n}}|\tilde{F}(\mathbf{n})|^{2}$.

\subsection{Grids on the fundamental domain $F_{e}$}

In order to determine an analogue of the finite Fourier transform, based on $E$-orbit functions, we need an analogue of the set

$$
\left\{\mathbf{m}=\left\{m_{1}, m_{2}, \ldots, m_{n}\right\} \mid m_{i} \in\{1,2, \ldots, N\}\right\},
$$

used for multidimensional finite Fourier transform. Such a set has to be invariant with respect to the even Weyl group $W_{e}$ (see [11]).

We know that the coroot lattice $Q^{\vee}$ is a discrete $W$-invariant subset of $E_{n}$. Clearly, the set $\frac{1}{m} Q^{\vee}$ is also $W$-invariant, where $m$ is a fixed positive integer. Then the set

$$
T_{m}=\frac{1}{m} Q^{\vee} / Q^{\vee}
$$


is finite and $W$-invariant. If $\alpha_{1}, \alpha_{2}, \ldots, \alpha_{l}$ is the set of simple root for the Weyl group $W$, then $T_{m}$ can be identified with the set of elements

$$
m^{-1} \sum_{i=1}^{l} d_{i} \alpha_{i}^{\vee}, \quad d_{i}=0,1,2, \ldots, m-1 .
$$

We select from $T_{m}$ the set of elements which belongs to the closure $\bar{F}_{e}$ of the fundamental domain $F_{e}$. These elements lie in the collection $\frac{1}{m} Q^{\vee} \cap \bar{F}_{e}$.

Let $\mu \in \frac{1}{m} Q^{\vee} \cap \bar{F}_{e}$ be an element determining an element of $T_{m}$ and let $M$ be the least positive integer such that $M \mu \in P^{\vee}$. Then there exists the least positive integer $N$ such that $N \mu \in Q^{\vee}$. One has $M \mid N$ and $N \mid m$.

The collection of points of $T_{m}$ belonging to $\bar{F}$ (we denote the set of these points by $F_{M}$ ), where $F$ is the fundamental domain of the Weyl group $W$, coincides with the set of elements

$$
s=\frac{s_{1}}{M} \omega_{1}^{\vee}+\cdots+\frac{s_{l}}{M} \omega_{l}^{\vee}, \quad \omega_{i}^{\vee}=\frac{2 \omega_{i}}{\left\langle\alpha_{i}, \alpha_{i}\right\rangle},
$$

where $s_{1}, s_{2}, \ldots, s_{l}$ runs over values from $\{0,1,2, \ldots\}$ and satisfy the following condition: there exists a non-negative integer $s_{0}$ such that

$$
s_{0}+\sum_{i=1}^{l} s_{i} m_{i}=M,
$$

where $m_{1}, m_{2}, \ldots, m_{l}$ are non-negative integers from formula (2.8) (see [11]). (Values of $m_{i}$ for all simple Lie algebras can be found in Subsection 2.4.)

To every positive integer $M$ there corresponds the grid $F_{M}$ of points $(9.6)$ in $\bar{F}$ which corresponds to some set $T_{m}$ such that $M \mid m$. The precise relation between $M$ and $m$ can be defined by the grid $F_{M}$ (see [10]) . Acting upon the grid $F_{M}$ by elements of the Weyl group $W$ we obtain the whole set $T_{m}$. Below, we are interested in grids $F_{M}$ and do not need the corresponding numbers $m$.

For studying finite $E$-orbit function transforms we need grids $F_{M}^{e}$ such that $T_{m}$ is obtained by action by elements of $W_{e}^{\text {aff }}$. In order to obtain $F_{M}^{e}$ we fix a positive root $\alpha$ and construct the the set $F_{M} \cup r_{\alpha} F_{M}$. The set $F_{M} \cap r_{\alpha} F_{M}$ can be non-empty. Taking each point from $F_{M} \cup r_{\alpha} F_{M}$ only once we obtain the grid $F_{M}^{e}$. The set $\cup_{w \in W_{e}}$ aff $w F_{M}^{e}$ coincides with $T_{m}$, where some points are taken several times. The set $F_{M}^{e}$ depends on a choice of a root $\alpha$.

\subsection{Grids $F_{M}^{e}$ for $A_{2}, C_{2}$ and $G_{2}$}

In this section we give some examples of grids $F_{M}^{e}$ for the rank two cases (see [9]). Since the long root $\xi$ of $A_{2}$ is representable in the form $\xi=\alpha_{1}+\alpha_{2}$, where $\alpha_{1}$ and $\alpha_{2}$ are simple roots, that is, $m_{1}=m_{2}=1$ (see formula (9.7)), then

$$
F_{M}\left(A_{2}\right)=\left\{\frac{s_{1}}{M} \omega_{1}+\frac{s_{2}}{M} \omega_{2} ; s_{0}+s_{1}+s_{2}=M, s_{0}, s_{1}, s_{2} \in \mathbb{Z}^{\geq 0}\right\} .
$$

A direct computation shows that in the $\omega$-coordinates we have

$$
F_{2}\left(A_{2}\right)=\left\{(0,0),(1,0),(0,1),\left(\frac{1}{2}, 0\right),\left(0, \frac{1}{2}\right),\left(\frac{1}{2}, \frac{1}{2}\right)\right\} .
$$

We take a root $\alpha$ coinciding with the first simple root. Then

$$
r_{\alpha} F_{2}\left(A_{2}\right)=\left\{(0,0),(-1,1),(0,1),\left(-\frac{1}{2}, \frac{1}{2}\right),\left(0, \frac{1}{2}\right),\left(-\frac{1}{2}, 1\right) .\right\} .
$$

Thus, the grid $F_{2}^{e}\left(A_{2}\right)$ consists of different points from $F_{2}\left(A_{2}\right) \cup r_{\alpha} F_{2}\left(A_{2}\right)$ (9 points). 
For $F_{3}\left(A_{2}\right)$ we have

$$
F_{3}\left(A_{2}\right)=\left\{(0,0),(1,0),(0,1),\left(\frac{1}{3}, 0\right),\left(0, \frac{1}{3}\right),\left(\frac{2}{3}, 0\right),\left(0, \frac{2}{3}\right),\left(\frac{2}{3}, \frac{1}{3}\right),\left(\frac{1}{3}, \frac{2}{3}\right),\left(\frac{1}{3}, \frac{1}{3}\right)\right\} .
$$

Therefore,

$$
r_{\alpha} F_{3}\left(A_{2}\right)=\left\{(0,0),(-1,1),(0,1),\left(-\frac{1}{3}, \frac{1}{3}\right),\left(0, \frac{1}{3}\right),\left(-\frac{2}{3}, \frac{2}{3}\right),\left(0, \frac{2}{3}\right),\left(-\frac{2}{3}, 1\right),\left(-\frac{1}{3}, 1\right),\left(-\frac{1}{3}, \frac{2}{3}\right)\right\}
$$

and $F_{3}^{e}\left(A_{2}\right)$ consists of 16 points.

Since the long root $\xi$ of $C_{2}$ is representable in the form $\xi=2 \alpha_{1}+\alpha_{2}$, where $\alpha_{1}$ and $\alpha_{2}$ are simple roots, that is, $m_{1}=2, m_{2}=1$, then

$$
F_{M}\left(C_{2}\right)=\left\{\frac{s_{1}}{M} \omega_{1}^{\vee}+\frac{s_{2}}{M} \omega_{2}^{\vee} ; s_{0}+2 s_{1}+s_{2}=M, s_{0}, s_{1}, s_{2} \in \mathbb{Z}^{\geq 0}\right\} .
$$

A direct computation shows that in the $\omega^{\vee}$-coordinates we have

$$
\begin{aligned}
& F_{2}^{e}\left(C_{2}\right)=\left\{(0,0),(0,1),\left(\frac{1}{2}, 0\right),\left(0, \frac{1}{2}\right),\left(-\frac{1}{2}, \frac{1}{2}\right)\right\} \\
& F_{3}^{e}\left(C_{2}\right)=\left\{(0,0),(0,1),\left(\frac{1}{3}, 0\right),\left(0, \frac{1}{3}\right),\left(0, \frac{2}{3}\right),\left(\frac{1}{3}, \frac{1}{3}\right),\left(-\frac{1}{3}, \frac{1}{3}\right),\left(-\frac{1}{3}, \frac{2}{3}\right)\right\} .
\end{aligned}
$$

Since the long root $\xi$ of $G_{2}$ is representable in the form $\xi=2 \alpha_{1}+3 \alpha_{2}$, where $\alpha_{1}$ and $\alpha_{2}$ are simple roots, that is, $m_{1}=2, m_{2}=3$, then

$$
F_{M}\left(G_{2}\right)=\left\{\frac{s_{1}}{M} \omega_{1}^{\vee}+\frac{s_{2}}{M} \omega_{2}^{\vee} ; s_{0}+2 s_{1}+3 s_{2}=M, s_{0}, s_{1}, s_{2} \in \mathbb{Z}^{\geq 0}\right\} .
$$

A computation shows that in the $\omega^{\vee}$-coordinates we have

$$
\begin{aligned}
& F_{2}^{e}\left(G_{2}\right)=\{(0,0),(1,0),(-1,3)\}, \\
& F_{3}^{e}\left(G_{2}\right)=\left\{(0,0),\left(0, \frac{1}{3}\right),\left(\frac{1}{3}, 0\right),\left(-\frac{1}{3}, 1\right)\right\}, \\
& F_{4}^{e}\left(G_{2}\right)=F_{2}^{e}\left(G_{2}\right) \bigcup\left\{\left(\frac{1}{4}, 0\right),\left(0, \frac{1}{4}\right),\left(-\frac{1}{4}, \frac{3}{4}\right\},\right. \\
& F_{5}^{e}\left(G_{2}\right)=\left\{(0,0),\left(0, \frac{1}{5}\right),\left(\frac{1}{5}, 0\right),\left(\frac{1}{5}, \frac{1}{5}\right),\left(\frac{2}{5}, 0\right),\left(-\frac{1}{5}, \frac{3}{5}\right),\left(-\frac{1}{5}, \frac{4}{5}\right),\left(-\frac{2}{5}, \frac{6}{5}\right)\right\}, \\
& F_{8}^{e}\left(G_{2}\right)=F_{4}^{e}\left(G_{2}\right) \bigcup\left\{\left(\frac{1}{8}, 0\right),\left(0, \frac{1}{8}\right),\left(\frac{1}{8}, \frac{1}{8}\right),\left(\frac{1}{4}, \frac{1}{8}\right),\left(-\frac{1}{8}, \frac{3}{8}\right),\left(-\frac{1}{8}, \frac{1}{2}\right),\left(-\frac{1}{4}, \frac{7}{8}\right)\right\} .
\end{aligned}
$$

\subsection{Expansion in $E$-orbit functions through expansion on grids}

Let us give an analogue of the finite Fourier transform when instead of exponential functions we use $E$-orbit functions. This analogue is not so simple as finite Fourier transform. It is called the finite $E$-orbit function transform. This transform is used in order to be able to recover (at least approximately) the expansion $f(x)=\sum_{\lambda} a_{\lambda} E_{\lambda}(x)$ for continuous values of $x$ by values of $f(x)$ on a finite set of point.

Under considering the finite Fourier transform in Section 9.1, we have restricted the exponential function to a discrete set. Similarly, in order to determine finite transform, based on $E$-orbit functions, we have to restrict $E$-orbit functions $E_{\lambda}(x)$ to appropriate finite sets of values of $x$. Candidates for such finite sets are sets $T_{m}$. However, $E$-orbit functions $E_{\lambda}(x)$ with integral $\lambda$ are invariant with respect to the affine even Weyl group $W_{e}^{\text {aff }}$. For this reason, we consider $E$-orbit functions $E_{\lambda}(x)$ on grids $F_{M}^{e}$, which are parts of the sets $T_{m}$.

On the other side, we have also to choose a finite number of $E$-orbit functions, that is, a finite number of integral $\lambda \in P_{+}^{e}$. The best choose is when a number of $E$-orbit functions coincides with the number $\left|F_{M}^{e}\right|$ of elements in $F_{M}^{e}$. These $E$-orbit functions must be selected in such a way that the matrix

$$
\left(E_{\lambda_{i}}\left(x_{j}\right)\right)_{\lambda_{i} \in \Omega, x_{j} \in F_{M}}
$$


(where $\Omega$ is our finite set of integral elements $\lambda$ ) is not singular. In order to have non-singularity of this matrix some conditions must be satisfied. In general, they are not known. For this reason, we consider some, more weak, form of the transform (when $|\Omega| \geq\left|F_{M}^{e}\right|$ ) and then explain how the set $|\Omega|$ of $\lambda \in P_{+}^{e}$ can be chosen in such a way that $|\Omega|=\left|F_{M}^{e}\right|$.

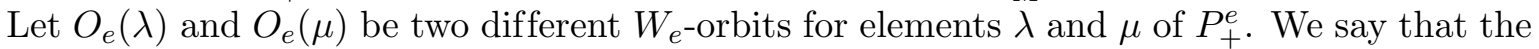
group $T_{m}$ separates $O_{e}(\lambda)$ and $O_{e}(\mu)$ if for any two elements $\lambda_{1} \in O_{e}(\lambda)$ and $\mu_{1} \in O_{e}(\mu)$ there exists an element $x \in T_{m}$ such that $\exp \left(2 \pi \mathrm{i}\left\langle\lambda_{1}, x\right\rangle\right) \neq \exp \left(2 \pi \mathrm{i}\left\langle\mu_{1}, x\right\rangle\right)$. Note that $\lambda$ may coincide with $\mu$.

Let $f_{1}$ and $f_{2}$ be two functions on the Euclidean space $E_{n}$ which are finite linear combinations of $E$-orbit functions. We introduce a $T_{m}$-scalar product for $f_{1}$ and $f_{2}$ by the formula

$$
\left\langle f_{1}, f_{2}\right\rangle_{T_{m}}=\sum_{x \in T_{m}} f_{1}(x) \overline{f_{2}(x)}
$$

Then the following proposition is true (see [11] and [10]):

Proposition 7. If $T_{m}$ separates the orbits $O_{e}(\lambda)$ and $O_{e}(\mu), \lambda, \mu \in P_{+}^{e}$, then

$$
\left\langle E_{\lambda}, E_{\mu}\right\rangle_{T_{m}}=m^{n}\left|O_{e}(\lambda)\right| \delta_{\lambda \mu} .
$$

Proof. We have

$$
\begin{aligned}
\left\langle E_{\lambda}, E_{\mu}\right\rangle_{T_{m}} & =\sum_{x \in T_{m}} \sum_{\sigma \in O_{e}(\lambda)} \sum_{\tau \in O_{e}(\mu)} \exp (2 \pi \mathrm{i}\langle\sigma-\tau, x\rangle) \\
& =\sum_{\sigma \in O_{e}(\lambda)} \sum_{\tau \in O_{e}(\mu)}\left(\sum_{x \in T_{m}} \exp (2 \pi \mathrm{i}\langle\sigma-\tau, x\rangle)\right) .
\end{aligned}
$$

Since $T_{m}$ separates $O_{e}(\lambda)$ and $O_{e}(\mu)$, then none of the differences $\sigma-\tau$ in the last sum vanishes on $T_{m}$. Since $T_{m}$ is a group and $\left|T_{m}\right|=m^{n}$, one has

$$
\sum_{x \in T_{m}} \exp (2 \pi \mathrm{i}\langle\sigma-\tau, x\rangle)=m^{n} \delta_{\sigma, \tau}
$$

Therefore, $\left\langle E_{\lambda}, E_{\mu}\right\rangle_{T_{m}}=m^{n}\left|O_{e}(\lambda)\right| \delta_{\lambda \mu}$. The proposition is proved.

Let $f$ be an invariant (with respect to $W_{e}^{\text {aff }}$ ) function on the Euclidean space $E_{n}$ which is a finite linear combination of $E$-orbit functions:

$$
f(x)=\sum_{\lambda_{j} \in P_{+}^{e}} a_{\lambda_{j}} E_{\lambda_{j}}(x) .
$$

Our aim is to determine $f(x)$ by its values on a finite subset of $E_{n}$, namely, on $T_{m}$.

We suppose that $T_{m}$ separate orbits $O_{e}\left(\lambda_{j}\right)$ with $\lambda_{j}$ from the right hand side of (9.10). Then taking the $T_{m}$-scalar product of both sides of (9.10) with $E_{\lambda_{j}}$ and using the relation (9.9) we obtain

$$
a_{\lambda_{j}}=\left(m^{n}\left|O_{e}\left(\lambda_{j}\right)\right|\right)^{-1}\left\langle f, E_{\lambda_{j}}\right\rangle_{T_{m}} .
$$

Let now $s^{(1)}, s^{(2)}, \ldots, s^{(h)}$ be all elements of $\bar{F}_{e} \cap \frac{1}{m} Q^{\vee}$. By $W_{s^{(i)}}$ we denote the subgroup of $W_{e}$ whose elements leave $s^{(i)}$ invariant. Then

$$
a_{\lambda_{j}}=m^{-n}\left|O_{e}\left(\lambda_{j}\right)\right|^{-1} \sum_{x \in T_{m}} f(x) \overline{E_{\lambda_{j}}(x)}=m^{-n}\left|W_{\lambda_{j}}\right| \sum_{i=1}^{h}\left|W_{s^{(i)}}\right|^{-1} f\left(s^{(i)}\right) \overline{\varphi_{\lambda_{j}}\left(s^{(i)}\right)} .
$$


Thus, the finite number of values $f\left(s^{(i)}\right), i=1,2, \ldots, h$, of the function $f(x)$ determines the coefficients $a_{\lambda_{j}}$ and, therefore, determines the function $f(x)$ on the whole space $E_{n}$.

This means that we can reconstruct a $W_{e}^{\text {aff }}$-invariant function $f(x)$ on the whole Euclidean space $E_{n}$ by its values on the finite set $F_{M}$ under an appropriate value of $M$. Namely, we have to expand this function, taken on $F_{M}$, into the series (9.10) by means of the coefficients $a_{\lambda_{j}}$, determined by formula (9.11), and then to continue analytically the expansion (9.10) to the whole fundamental domain $F_{e}$ (and, therefore, to the whole space $E_{n}$ ), that is, to consider the decomposition (9.10) for all $x \in E_{n}$.

We have assumed that the function $f(x)$ is a finite linear combination of $E$-orbit functions. If $f(x)$ expands into infinite sum of orbit functions, then for applying the above procedure we have to approximate the function $f(x)$ by taking a finite number of terms in this infinite sum and then to apply the procedure. That is, in this case we obtain an approximate expression of the function $f(x)$ by using a finite number of its values.

At last, we explain how to choose a set $\Omega$ in formula (9.8). The set $F_{M}$ consists of the points (9.6). These points determines the set $\Xi$ of points

$$
\lambda=s_{1} \omega_{1}+s_{2} \omega_{2}+\cdots+s_{l} \omega_{l},
$$

where $s_{1}, s_{2}, \ldots, s_{l}$ run over the same values as for the set $F_{M}$. The set $\Xi \cup r_{\alpha} \Xi$, where each point is taken only once, can be taken as the set $\Omega$ (see [10]).

\section{$10 W_{e}$-symmetric functions}

$E$-orbit functions are symmetrized versions of the exponential function, when symmetrization is fulfilled by an even Weyl group $W_{e}$. Instead of the exponential function we can take any other set of functions, for example, a set of orthogonal polynomials or a countable set of functions. Then we obtain a corresponding set of orthogonal $W_{e^{-}}$symmetric polynomials or a set of $W_{e^{-}}$symmetric functions. Such sets of polynomials and functions are considered in this section.

\subsection{Symmetrization by $E$-orbit functions}

$E$-orbit functions can be used for obtaining $W_{e}$-symmetric sets of functions. Let $u_{m}(x), m=$ $0,1,2, \ldots$, be a set of continuous functions of one variables. We create functions of $n$ variables

$$
u_{i_{1}, i_{2}, \ldots, i_{n}}\left(x_{1}, x_{2}, \ldots, x_{n}\right) \equiv u_{i_{1}}\left(x_{1}\right) u_{i_{2}}\left(x_{2}\right) \cdots u_{i_{n}}\left(x_{n}\right), \quad i_{k}=0,1,2, \ldots
$$

Then the functions

$$
\tilde{u}_{i_{1}, i_{2}, \ldots, i_{n}}\left(\lambda_{1}, \lambda_{2}, \ldots, \lambda_{n}\right)=\int_{F_{e}} u_{i_{1}, i_{2}, \ldots, i_{n}}\left(x_{1}, x_{2}, \ldots, x_{n}\right) E_{\lambda}\left(x_{1}, x_{2}, \ldots, x_{n}\right) d x,
$$

where $\lambda \equiv\left(\lambda_{1}, \lambda_{2}, \ldots, \lambda_{n}\right), E_{\lambda}(x)$ is a $E$-orbit function, and $d x$ is the Euclidean measure on $E_{n}$ (that is, $d x=d x_{1} \cdots d x_{n}$ ), is symmetric with respect to the action of the even Weyl group $W_{e}$. Indeed, for $w \in W_{e}$ we have

$$
\begin{aligned}
\tilde{u}_{i_{1}, i_{2}, \ldots, i_{n}}(w \lambda) & =\int_{F_{e}} u_{i_{1}, i_{2}, \ldots, i_{n}}\left(x_{1}, x_{2}, \ldots, x_{n}\right) E_{w \lambda}\left(x_{1}, x_{2}, \ldots, x_{n}\right) d x \\
& =\int_{F_{e}} u_{i_{1}, i_{2}, \ldots, i_{n}}\left(x_{1}, x_{2}, \ldots, x_{n}\right) E_{\lambda}\left(x_{1}, x_{2}, \ldots, x_{n}\right) d x=\tilde{u}_{i_{1}, i_{2}, \ldots, i_{n}}(\lambda) .
\end{aligned}
$$

Formula (10.1) is used for obtaining $W_{e}$-symmetric functions or polynomials.

If $u_{m}(x), m=0,1,2, \ldots$, are orthogonal functions, then the functions (10.1), taken for $i_{1} \geq i_{2} \geq \cdots \geq i_{n}$, constitute a set of $W_{e}$-symmetric orthogonal functions on the domain $D_{+}^{e}$. 


\subsection{Eigenfunctions of $E$-orbit function transform for $W_{e}\left(A_{n}\right)$}

Let $H_{n}(x), n=0,1,2, \ldots$, be the well-known Hermite polynomials. They are defined by the formula

$$
H_{n}(x)=n ! \sum_{m=0}^{[n / 2]} \frac{(-1)^{m}(2 x)^{n-2 m}}{m !(n-2 m) !},
$$

where $[n / 2]$ is an integral part of the number $n / 2$. They satisfy the relation

$$
\frac{1}{\sqrt{2 \pi}} \int_{-\infty}^{\infty} e^{\mathrm{i} p x} e^{-p^{2} / 2} H_{m}(p) d p=\mathrm{i}^{-m} e^{-x^{2} / 2} H_{m}(x)
$$

(see, for example, Subsection 12.2.4 in [30]), which can be written in the form

$$
\int_{-\infty}^{\infty} e^{2 \pi \mathrm{i} p x} e^{-\pi p^{2}} H_{m}(\sqrt{2 \pi} p) d p=\mathrm{i}^{m} e^{-\pi x^{2}} H_{m}(\sqrt{2 \pi} x) .
$$

This relation shows that the function $e^{-\pi p^{2}} H_{m}(\sqrt{2 \pi} p)$ is an eigenfunction of the Fourier transform of one variable with eigenvalue $\mathrm{i}^{m}$.

Using the Hermite polynomials we create polynomials of many variables

$$
H_{\mathbf{m}}(\mathbf{x}) \equiv H_{m_{1}, m_{2}, \ldots, m_{n}}\left(x_{1}, x_{2}, \ldots, x_{n}\right):=H_{m_{1}}\left(x_{1}\right) H_{m_{2}}\left(x_{2}\right) \cdots H_{m_{n}}\left(x_{n}\right) .
$$

The functions

$$
e^{-|\mathbf{x}|^{2} / 2} H_{\mathbf{m}}(\mathbf{x}), \quad m_{i}=0,1,2, \ldots, \quad i=1,2, \ldots, n,
$$

form an orthogonal basis of the Hilbert space $L^{2}\left(\mathbb{R}^{n}\right)$ with the scalar product

$$
\left\langle f_{1}, f_{2}\right\rangle:=\int_{\mathbb{R}^{n}} f_{1}(\mathbf{x}) \overline{f_{2}(\mathbf{x})} d \mathbf{x},
$$

where $d \mathbf{x}=d x_{1} d x_{2} \cdots d x_{n}$.

We make $W_{e}$-symmetrization of the functions

$$
e^{-\pi|x|} H_{\mathbf{m}}(\sqrt{2 \pi} \mathbf{x}), \quad m_{i}=0,1,2, \ldots,
$$

(obtained from (10.4) by replacing $\mathbf{x}$ by $\sqrt{2 \pi} \mathbf{x}$ ) by means of $E$-orbit functions of $A_{n-1}$ :

$$
\int_{\mathbb{R}^{n}} E_{\lambda}(\mathbf{x}) e^{-\pi|\mathbf{x}|^{2}} H_{\mathbf{m}}(\sqrt{2 \pi} \mathbf{x})=\mathrm{i}^{|\mathbf{m}|} e^{-\pi|\lambda|^{2}} \mathcal{H}_{\mathbf{m}}(\sqrt{2 \pi} \lambda)
$$

where $E_{\lambda}(\mathbf{x})$ is an $E$-orbit function of $A_{n-1}$ and $\lambda=\left(\lambda_{1}, \lambda_{2}, \ldots, \lambda_{n}\right)$.

The polynomials $\mathcal{H}_{\mathbf{m}}$ are symmetric with respect to the even Weyl group $W_{e} \equiv S_{n} / S_{2}:=S_{n}^{e}$ of $A_{n-1}$ :

$$
\mathcal{H}_{\mathbf{m}}(w \lambda)=\mathcal{H}_{\mathbf{m}}(\lambda), \quad \mathcal{H}_{w \mathbf{m}}(\lambda)=\mathcal{H}_{\mathbf{m}}(\lambda), \quad w \in S_{n}^{e} .
$$

For this reason, $\mathcal{H}_{\mathbf{m}}(\lambda)$ can be considered for values of $\lambda=\left(\lambda_{1}, \lambda_{2}, \ldots, \lambda_{n}\right)$ such that $\lambda_{1} \geq \lambda_{2} \geq$ $\cdots \geq \lambda_{n}$.

The polynomials $\mathcal{H}_{\mathbf{m}}$ are of the form

$$
\mathcal{H}_{\mathbf{m}}(\lambda)=\sum_{w \in S_{n}^{e}} H_{w \mathbf{m}}(\lambda)
$$

where the polynomials $H_{w \mathbf{m}}(\lambda)$ are of the form (10.3). 
Now we apply $E$-orbit function transform (8.7) (we denote this transform by $\mathfrak{F}$ ) to the $W_{e^{-}}$ symmetric function (10.6). Taking into account formula (10.5) we obtain

$$
\begin{aligned}
\mathfrak{F}\left(e^{-\pi|\mathbf{x}|^{2}} \mathcal{H}_{\mathbf{m}}(\sqrt{2 \pi} \mathbf{x})\right): & \frac{2}{\left|S_{n}\right|} \int_{\mathbb{R}^{n}} E_{\lambda}(\mathbf{x}) e^{-\pi|\mathbf{x}|^{2}} \mathcal{H}_{\mathbf{m}}(\sqrt{2 \pi} \mathbf{x}) d \mathbf{x} \\
& =\mathrm{i}^{|\mathbf{m}|} e^{-\pi|\lambda|^{2}} \mathcal{H}_{\mathbf{m}}(\sqrt{2 \pi} \lambda),
\end{aligned}
$$

where $\left|S_{n}\right|$ is an order of the permutation group $S_{n}$, that is, functions (10.6) are eigenfunctions of the $E$-orbit function transform $\mathfrak{F}$. Since the functions (10.6) for $m_{i}=0,1,2, \ldots, i=1,2, \ldots, n$, $m_{1} \geq m_{2} \geq \cdots \geq m_{n}$, form an orthogonal basis of the Hilbert space $L_{\text {sym }}^{2}\left(\mathbb{R}^{n}\right)$ of functions from $L^{2}\left(\mathbb{R}^{n}\right)$ symmetric with respect to $W_{e}$, then they constitute a complete set of eigenfunctions of this transform. Thus, this transform has only four eigenvalues i, $-\mathrm{i}, 1,-1$ in $L_{\mathrm{sym}}^{2}\left(\mathbb{R}^{n}\right)$. This means that, as in the case of the usual Fourier transform, we have

$$
\mathfrak{F}^{4}=1
$$

\section{3 $W_{e}\left(A_{n}\right)$-symmetric sets of polynomials}

In the previous subsection we constructed $W_{e}$-symmetric sets of functions connected with Hermite polynomials. Other sets of orthogonal polynomials can be similarly constructed.

Let $p_{m}(x), m=0,1,2, \ldots$, be the set of orthogonal polynomials in one variable such that

$$
\int_{\mathbb{R}} p_{m}(x) p_{m^{\prime}}(x) d \sigma(x)=\delta_{m m^{\prime}}
$$

where $d \sigma(x)$ is some orthogonality measure, which may be continuous or discrete.

We create a set of symmetric polynomials of $n$ variables as follows:

$$
\begin{aligned}
& p_{\mathbf{m}}^{\mathrm{sym}}(\mathbf{x})=\sum_{w \in S_{n}^{e} / S_{\mathbf{m}}} p_{m_{w(1)}}\left(x_{1}\right) p_{m_{w(2)}}\left(x_{2}\right) \cdots p_{m_{w(n)}}\left(x_{n}\right), \\
& m_{i}=0,1,2, \ldots, \quad i=1,2, \ldots, n
\end{aligned}
$$

where $\mathbf{m}=\left(m_{1}, m_{2}, \ldots, m_{n}\right), m_{1} \geq m_{2} \geq \cdots \geq m_{n} \geq 0, \mathbf{x}=\left(x_{1}, x_{2}, \ldots, x_{n}\right)$, and $w(1), w(2)$, $\ldots, w(n)$ is a set of numbers $1,2, \ldots, n$ transformed by the permutation $w \in S_{n}^{e} / S_{\mathbf{m}}$, where $S_{\mathbf{m}}$ is the subgroup of $S_{n}$ consisting of elements leaving $\mathbf{m}$ invariant.

It is easy to check that the polynomials $p_{\mathbf{m}}^{\mathrm{sym}}(\mathbf{x})$ are symmetric with respect to transformations of $S_{n}^{e}$ :

$$
p_{\mathbf{m}}^{\mathrm{sym}}(w \mathbf{x})=p_{\mathbf{m}}^{\mathrm{sym}}(\mathbf{x}), \quad w \in S_{n} .
$$

Thus, we may consider the polynomials (10.7) on the closure of the fundamental domain of the transformation group $W_{e}\left(A_{n-1}\right) \equiv S_{n}^{e}$. This closure (which is denoted as $D_{+}^{e}$ ) coincides with the set of points $\mathbf{x}=\left(x_{1}, x_{2}, \ldots, x_{n}\right)$ for which

$$
x_{1}, x_{2} \geq \cdots \geq x_{n}
$$

The set of polynomials $(10.7)$ is orthogonal with respect to the product measure $d \sigma(\mathbf{x}) \equiv$ $d \sigma\left(x_{1}\right) d \sigma\left(x_{2}\right) \cdots d \sigma\left(x_{n}\right)$. Indeed, we have

$$
\int_{D_{+}^{e}} p_{\mathbf{m}}^{\mathrm{sym}}(\mathbf{x}) \overline{p_{\mathbf{m}^{\prime}}^{\mathrm{sym}}(\mathbf{x})} d \sigma(\mathbf{x})=\frac{\left|O_{e}(\mathbf{m})\right|}{\left|S_{n}^{e}\right|} \delta_{\mathbf{m m}^{\prime}}=\frac{1}{\left|S_{\mathbf{m}}\right|} \delta_{\mathbf{m m}^{\prime}}
$$

where $O_{e}(\mathbf{m})$ is the $S_{n}^{e}$-orbit of the point $\mathbf{m}$. 


\section{Acknowledgements}

The first author acknowledges CRM of University of Montreal for hospitality when this paper was under preparation. We are grateful for partial support for this work from the National Science and Engineering Research Council of Canada, MITACS, the MIND Institute of Costa Mesa, California, and Lockheed Martin, Canada.

\section{References}

[1] Patera J., Orbit functions of compact semisimple Lie groups as special functions, in Proceedings of Fifth International Conference "Symmetry in Nonlinear Mathematical Physics" (June 23-29, 2003, Kyiv), Editors A.G. Nikitin, V.M. Boyko, R.O. Popovych and I.A. Yehorchenko, Proceedings of Institute of Mathematics, Kyiv 50 (2004), Part 3, 1152-1160.

[2] Patera J., Compact simple Lie groups and their $C$-, $S$-, and E-transforms, SIGMA 1 (2005), 025, 6 pages, math-ph/0512029.

[3] Atoyan A., Patera J., Properties of continuous Fourier extension of the discrete cosine transform and its multidimensional generalization, J. Math. Phys. 45 (2004), 2468-2491, math-ph/0309039.

[4] Patera J., Zaratsyan A., Discrete and continuous cosine transform generalized to Lie groups $S U(2) \times S U(2)$ and $O(5)$, J. Math. Phys. 46 (2005), 053514, 25 pages.

[5] Patera J., Zaratsyan A., Discrete and continuous cosine transform generalized to Lie groups $S U(2)$ and $G_{2}$, J. Math. Phys. 46 (2005), 113506, 17 pages.

[6] Patera J., Zaratsyan A., Discrete and continuous sine transforms generalized to compact semisimple Lie groups of rank two, J. Math. Phys. 47 (2006), 043512, 22 pages.

[7] Klimyk A.U., Patera J., Orbit functions, SIGMA 2 (2006), 006, 60 pages, math-ph/0601037.

[8] Klimyk A.U., Patera J., Antisymmetric orbit functions, SIGMA 3 (2007), 023, 83 pages, math-ph/0702040.

[9] Kashuba I., Patera J., Discrete and continuous exponential transforms of simple Lie groups of rank two, J. Phys. A: Math. Theor. 40 (2007), 1751-1774, math-ph/0702016.

[10] Moody R.V., Patera J., Orthogonality within the families of $C$-, $S$-, and $E$-functions of any compact semisimple Lie group, SIGMA 2 (2006), 076, 14 pages, math-ph/0611020.

[11] Moody R.V., Patera J., Computation of character decompositions of class functions on compact semisimple Lie groups, Math. Comp. 48 (1987), 799-827.

[12] Klimyk A.U., Patera J., (Anti)symmetric multivariate exponential functions and corresponding Fourier transforms, J. Phys. A: Math. Theor. 40 (2007), 10473-10489, arXiv:0705.3572.

[13] Klimyk A.U., Patera J., (Anti)symmetric multivariate trigonometric functions and corresponding Fourier transforms, J. Math. Phys. 48 (2007), 093504, 24 pages, arXiv:0705.4186.

[14] Macdonald I.G., Symmetric functions and hall polynomials, 2nd ed., Oxford Univ. Press, Oxford, 1995.

[15] Macdonald I.G., A new class of symmetric functions, Publ. I.R.M.A. Strasbourg, 372/S-20, Actes 20 Séminaire Lotharingien, 1988, 131-171.

[16] Macdonald I.G., Orthogonal polynomials associated with root systems, Séminaire Lotharingien de Combinatoire, Actes B45a, Stracbourg, 2000.

[17] Vilenkin N.Ja., Klimyk A.U., Representations of Lie groups and special functions: recent advances, Kluwer, Dordrecht, 1995.

[18] Moody R.V., Patera J., Elements of finite order in Lie groups and their applications, in Proceedings of XIII Int. Colloq. on Group Theoretical Methods in Physics, Editor W. Zachary, World Scientific Publishers, Singapore, 1984, 308-318.

[19] McKay W.G., Moody R.V., Patera J., Tables of $E_{8}$ characters and decomposition of plethysms, in Lie Algebras and Related Topics, Editors D.J. Britten, F.W. Lemire and R.V. Moody, Amer. Math. Society, Providence RI, 1985, 227-264.

[20] McKay W.G., Moody R.V., Patera J., Decomposition of tensor products of $E_{8}$ representations, Algebras Groups Geom. 3 (1986), 286-328.

[21] Patera J., Sharp R.T., Branching rules for representations of simple Lie algebras through Weyl group orbit reduction, J. Phys. A: Math. Gen. 22 (1989), 2329-2340. 
[22] Grimm S., Patera J., Decomposition of tensor products of the fundamental representations of $E_{8}$, in Advances in Mathematical Sciences - CRM's 25 Years, Editor L. Vinet, CRM Proc. Lecture Notes, Vol. 11, Amer. Math. Soc., Providence, RI, 1997, 329-355.

[23] Rao K.R., Yip P., Discrete cosine transform - algorithms, advantages, applications, Academic Press, New York, 1990.

[24] Kane R., Reflection groups and invariants, Springer, New York, 2002.

[25] Humphreys J.E., Reflection groups and Coxeter groups, Cambridge Univ. Press, Cambridge, 1990.

[26] Humphreys J.E., Introduction to Lie algebras and representation theory, Springer, New York, 1972.

[27] Bremner M.R., Moody R.V., Patera J., Tables of dominant weight multiplicities for representations of simple Lie algebras, Marcel Dekker, New York, 1985.

[28] Kac V., Infinite dimensional Lie algebras, Birkhäuser, Basel, 1982.

[29] Mckay W.G., Patera J., Sannikoff D., The computation of branching rules for representations of semisimple Lie algebras, in Computers in Nonassociative Rings and Algebras, Editors R.E. Beck and B. Kolman, New York, Academic Press, 1977, 235-278.

[30] Vilenkin N.Ja., Klimyk A.U., Representations of Lie groups and special functions, Vol. 2, Kluwer, Dordrecht, 1993. 